

\section{DISCLAIMER}

This document was prepared as an account of work sponsored by an agency of the United States Government. Neither the United States Government nor the University of California nor any of their employees, makes any warranty, express or implied, or assumes any legal liability or responsibility for the accuracy, completeness, or usefulness of any information, apparatus, product, or process disclosed, or represents that its use would not infringe privately own rights. Reference herein to any specific commercial products, process, or service by trade name, trademark, manufacturer, or otherwise, does not necessarily constitute or imply its endorsement, recommendation, or favoring by the United States Government or the University of California. The views and opinions of authors expressed herein do not necessarily state or reflect those of the United States Government or the University of California, and shall not be used for advertising or product endorsement purposes.

This report has been reproduced directly from the best available copy.

Available to DOE and DOE contractors from the Office of Scientific and Technical Information P.O. Box 62, Oak Ridge, TN 37831

Prices available from (615) 576-8401, FTS 626-8401

Available to the public from the National Technical Information Service

U.S. Department of Commerce 5285 Port Royal Rd., Springfield, VA 22161 


\section{DISCLAIMER}

Portions of this document may be illegible in electronic image products. Images are produced from the best available original document. 


\title{
On The Numerical Treatment of Problems in Atmospheric Chemistry
}

\author{
Colin J. Aro \\ University of California, Davis
}

Doctor of Philosophy Thesis

Manuscript date: September 1995

LAWRENCE LIVERMORE NATIONAL LABORATORY

University of California $\bullet$ Livermore, California $\bullet 94551$

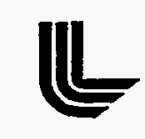




\title{
On the Numerical Treatment of Problems in Atmospheric Chemistry
}

\author{
by \\ Colin J. Aro \\ B.A. (University of California, Berkeley) 1988 \\ M.S. (California State University, Hayward) 1991 \\ DISSERTATION
}

Submitted in partial satisfaction of the requirements for the degree of

DOCTOR OF PHILOSOPHY

in

Engineering - Applied Science

in the

OFFICE OF GRADUATE STUDIES

of the

UNIVERSITY OF CALIFORNIA

DAVIS

Approved:

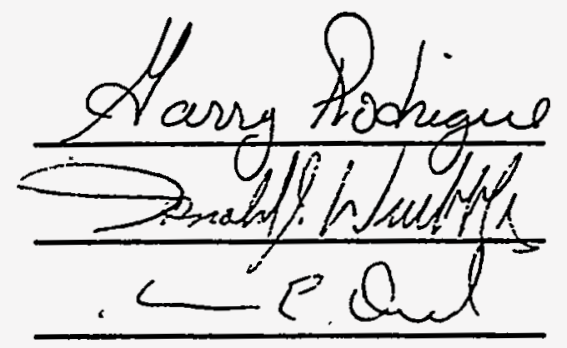

Committee in Charge

1995 
Colin Jeffry Aro

December 1995

Engineering - Applied Science

\title{
On the Numerical Treatment of Problems in Atmospheric Chemistry
}

\begin{abstract}
Atmospheric chemical-radiative-transport (CRT) models are vital in performing research on atmospheric chemical change. Even with the enormous computing capability delivered by massively parallel systems, extended three dimensional CRT simulations are still not computationally feasible. The major obstacle in a CRT model is the nonlinear ODE system describing the chemical kinetics in the model. These ODE systems are usually very stiff and account for anywhere from $75 \%$ to $90 \%$ of the CPU time required to run a CRT model. In this study, a simple explicit class of time stepping method is developed and demonstrated to be useful in treating chemical ODE systems without the use of a Jacobian matrix. These methods, called preconditioned time differencing methods, are tested on small mathematically idealized problems, box model problems, and full 2-D and 3-D CRT models. The methods are found to be both fast and memory efficient. Studies are performed on both vector and parallel systems. The preconditioned time differencing methods are established as a viable alternative to the more common backward differentiation formulas in terms of CPU speed across architectural platforms.
\end{abstract}




\section{Acknowledgments}

I would like to acknowledge the strong support of Garry Rodrigue, Doug

Rotman, and Don Wuebbles, whose daily guidance and support were vital to the success of my graduate study.

I would also like to thank several LLNL colleagues: Britton Chang, Peter

Connell, Keith Grant, Doug Kinnison, Tom Kuczmarski, Ann Orel, Ken

Patten (now at the University of Illinois), John Tamaresis, John Tannahill, and Ray Tarp for various technical contributions to this work. Special thanks go to Alan Hindmarsh for all his background on the many stiff ODE solvers and to Don Wolitzer, Dan Jurca, and Chris Morgan (all of CSU Hayward) for getting me here. Big special thanks to Shirley Davis for help with all of my manuscripts and other very important (and often thankless) tasks! Thanks also to a couple of student colleagues: Paul Amala and Kan Anant for everything from technical help to daily gripe sessions.

Many others deserve special mention: Bill Schaefer (wine at Natalie's?), John Bunker (flying anyone?), the brothers Paul (Rubinstein and Rothenberg), Mark Bender, Kevin Clevenger, John Cavanaugh, Marc Phillips and Jennifer Huggins, Pete Wardlow (I am the Polar Bear), Joy and Paul Barney, Mike Stevens (perpetually infected with the Lipschitz condition), Chris Hart, Bob and Aleda Barden, John and Melanie Allen (also Megan 
and Jeremy), Sherry and Ruben Maldonado (and now Joshua), Shawn Aro, and Stan Pisle (call me Dr. Aro for the rest of eternity, sucker). All played some role in the completion of this work.

I owe an immeasurable debt to my wife Yolanda for all of her love and support. For every person working on a dissertation there is a loved one waiting patiently for them to finish. Yolanda's encouragement and understanding always made difficult times much easier to live with.

This work was performed under the auspices of the U.S. Department of Energy by the Lawrence Livermore National Laboratory under contract number W-7405-Eng-48 and was supported in part by the NASA Atmospheric Chemistry Modeling and Analysis Program and the Department of Energy's Office of Health and Environmental Research, Environmental Science Division. 


\author{
Abstract ii \\ Acknowledgments iii
}

CHAPTER 1

Overview of Numerical ChemicalRadiative-Transport Modeling 1

Introduction 1

Standard Numerical Techniques for Conservation Equations 4

Methads for the General ODE Problem $S$

Stiff Systems of Ordinary Differential Equations 7

Basic Definition 8

Stability for Stiff Systems 13

The Problem With Implicitness 15

LSODE 16

Basic Language of Chemical Kinetics 18

Chemical Kinetics Equations 20

The Chapman Atmosphere 20

\title{
CHAPTER 2
}

\section{Preconditioned Time Differencing:}

\section{Development and Initial Results for}

Atmospheric Chemistry 23

Preconditioned Time Differencing 23

Chemical Kinetics Equations 27

More Complexity: Hydrogen Chemistry 49

The Box Model 56

Description 56

CHEMSODE 56

Timings 58

Results 59

Conclusions 66

CHAPTER 3 Preconditioned Time Differencing in Full CRT Models 67

Chemical-Radiative-Transport Models 67

3-D Model 67

Architectures 68 
IMPACT 69

2-D Model 86

Conclusions 100

CHAPTER 4

Conclusions and Future Direction 101

Summary: Results of this Study 101

Future Direction 103

CHAPTER 5

References 105

Appendix A

CHEMSODE 113 .

Code 113

Code usage and Example Problem 117 EXAMPLE 117

Appendix B

Chemical Mechanisms 121

The Box Model 121

3-D model 124

2-D Model 125 
CHAPTER 1

\section{Overview of Numerical Chemical- Radiative-Transport Modeling}

\subsection{Introduction}

Human activities are a major force affecting the chemical composition of the earth's atmosphere. The atmosphere is composed primarily of nitrogen (78.1\%) and oxygen (20.9\%) with many other trace gases found in far smaller quantities. Ozone $\left(\mathrm{O}_{3}\right)$ is an important example of a trace gas, existing in very small quantities but vital to life on Earth [36, 73, 74]. Ozone plays an important dual role in influencing the global climate. Its climactic effect depends on its distribution in both the troposphere and the stratosphere [73]. Ozone and molecular oxygen are the primary absorbers of UV and visible radiation in the atmosphere. Absorption by ozone in the stratosphere is responsible for the increase in temperature with altitude in that region of the atmosphere. Ozone also absorbs infrared radiation. Thus, increases in $\mathrm{O}_{3}$ above $30 \mathrm{~km}$ tend to decrease surface temperature, while increases below $30 \mathrm{~km}$ tend to increase surface temperature [36A].

The concentrations of other important trace gases are also changing $[36,43,68,69,73,74$, 75]. The most significant of these gases, with respect to their effect on the global ozone distribution and the global temperature structure, are carbon dioxide $\left(\mathrm{CO}_{2}\right)$, methane $\left(\mathrm{CH}_{4}\right)$, nitrous oxide $\left(\mathrm{N}_{2} \mathrm{O}\right)$, and the various chlorocarbons ( $\mathrm{ClC}$ 's). The concentrations 
of the chlorocarbons have, in recent decades, experienced the largest relative changes, a phenomenon due almost completely to anthropogenic sources [36]. Annual rates of change for all of the aforementioned gases are: $\mathrm{CO}_{2}(+0.4 \%), \mathrm{CH}_{4}(+1-2 \%), \mathrm{N}_{2} \mathrm{O}(+0.2-$ 0.3\%), $\mathrm{CFCl}_{3}(+5 \%), \mathrm{CF}_{2} \mathrm{Cl}_{2}(+5 \%), \mathrm{CCl}_{4}(+1 \%), \mathrm{CH}_{3} \mathrm{CCl}_{3}(+5 \%)^{*}$. [68, 69, 74] The chemical and physical effects that variations in these trace gases have on ozone distribution, temperature profile, and the global climate has been the subject of increasing investigation. $[16,18,31,32,36,43,68,69,70,72,73,74,75]$

Of great importance in conducting research on the changes occurring to ozone are numerical chemical-radiative-transport (CRT) models of the global atmosphere. $[13,36,43,72]$ At the heart these models are large sets of highly stiff, coupled, nonlinear partial differential equations of the advection-diffusion-reaction type. For example, as treated in the typical zonally averaged two dimensional model of the global atmosphere, the equations are [36]:

$$
\frac{\partial y_{i}}{\partial t}+\bar{v} \frac{\partial y_{i}}{\partial x}+\bar{w} \frac{\partial y_{i}}{\partial z}=R_{i}(y, t)+\frac{1}{\rho \cos \phi}\left(\frac{\partial}{\partial x}\right)\left(\rho K_{x x} \cos \phi \frac{\partial y_{i}}{\partial x}\right)+\frac{1}{\rho}\left(\frac{\partial}{\partial z}\right)\left(\rho K_{z z} \frac{\partial y_{i}}{\partial z}\right)
$$

where

- $y$ is a vector of zonally averaged mixing ratios $\left(y_{i}\right.$ being the mixing ratio for the ith chemical species), that is the average value of the mixing ratio over a spatial zone.

- $x$ and $z$ are the horizontal and vertical directions, respectively.

- $\bar{v}$ and $\bar{w}$ are the horizontal and vertical wind fields, respectively.

- $t$ is the time.

- $R_{i}(y, t)$ is the reactive portion of the system (i.e. a nonlinear function describing the actions of the chemical sources and sinks in the model).

- $p$ is the air density.

*. Until very recently. The increases in ClC concentrations have slowed due to control measures. 
- $\phi$ is the latitude.

On the left hand side of the equation is the time derivative of the zonally averaged mixing ratio together with the advective part of the transport (the vertical and horizontal winds). The right hand side has chemical sources and sinks together with diffusive terms that represent the effects of wave activity in the atmosphere together with sub-grid scale eddy transport. The constants $K_{x x}$, and $K_{z z}$ parameterize this eddy transport and vary between the troposphere and the stratosphere [36]. Note that the reactive term is generally nonlinear, and couples the mixing ratios of the various chemical species. The spatial domain is typically a two dimensional atmosphere ranging from pole to pole (in latitude) and from the surface of the earth to at least 50 kilometers (in altitude), the top of the stratosphere. This equation represents the basic chemical mass conservation requirement for each chemical species.

In addition to Eq. (1), there are also conservation equations for the atmosphere as a whole. These are the basic conservation equations for a fluid flow $[6,42]$ and take the form (shown in 1-D for simplicity):

$$
\frac{\partial}{\partial t} y(x, t)+\frac{\partial}{\partial x} f(y(x, t))=0 .
$$

These general conservation equations usually contain terms representing forces due to the fluid's resistance to shear stress. Viscosity is a measure of this resistance to shear stress and is considered negligible in the atmosphere. In this case, the equations for the conservation of mass, momentum and energy take the form [42]:

$$
\frac{\partial}{\partial t}\left[\begin{array}{c}
\rho \\
\rho v \\
E
\end{array}\right]+\frac{\partial}{\partial x}\left[\begin{array}{c}
\rho v \\
\rho v^{2}+P \\
v(E+P)
\end{array}\right]=0
$$

where $\rho$ is the total density, $v$ is the velocity, $E$ is the total energy, and $P$ is the pressure. The pressure is determined by an equation of state relation with the other variables $[6,42]$. 
Since the total density can be expressed as a weighted sum of the mixing ratios of the individual species $[6,36]$, the variable $\rho$ is also a function of the $y_{i}$ in the system (1). Thus, Eq. (3) is coupled into Eq. (1) and together they describe the atmosphere as a reactive flow problem [6].

\subsection{Standard Numerical Techniques for Conservation Equations}

The three standard methodologies for numerically solving PDE problems are finite difference $[6,25,37,42,47]$, finite element (or finite volume) $[14,62]$, and particle $[40,41]$ methods. For many atmospheric simulations, finite difference is the methodology of choice $[2,6,13,43,48,56,64]$.

In finite differencing, the domain is discretized by choosing a mesh width $\Delta x$ and a timestep $\Delta t$. The finite difference methods then produce approximations $y_{j}^{n}$ to the solu- . tions $y\left(x_{j}, t_{n}\right)$ at the discrete grid points via a Taylor series expansion. Various finite difference methods are designed by replacing derivatives with different finite difference approximations. Some of the more basic examples include the backward Euler, one-sided, Lax-Friedrichs, leapfrog, Lax-Wendroff, and beam-warming techniques [42]. More advanced methods, such as the flux corrected transport (FCT) methods used for advection in this study, are also commonly used $[48,56,64]$.

An important technique which is widely used in the context of reactive flow problems is the splitting $[15,42,60]$ principle. Splitting can be illustrated by considering the PDE

$$
\frac{\partial u}{\partial t}+a \frac{\partial u}{\partial x}+b \frac{\partial u}{\partial y}=0
$$

Splitting (or operator splitting) allows the solution of two 1-D problems in place of the original 2-D problem [42], treating each operator separately:

$$
\frac{\partial \hat{u}}{\partial t}+a \frac{\partial \hat{u}}{\partial x}=0
$$




$$
\frac{\partial \tilde{u}}{\partial t}+b \frac{\partial \tilde{u}}{\partial y}=0
$$

Operator splitting finds wide application in atmospheric modeling by separating the reaction operator from the advective-diffusive operators in the chemical mass conservation equation (1). Although splitting produces numerical results that are well within accuracy requirements, it is not well understood in a mathematically rigorous sense. This disadvantage is far outweighed by the advantage of treating the operators with techniques specially tailored to each operator's salient features.

This study will focus on the solution of the chemical kinetic portion of Eq. (1). This operator is split from the transport processes which are solved separately $[56,64]$. This results in a system of nonlinear stiff ODEs describing the kinetics in each zone:

$$
\frac{d y}{d t}=R(y, t)
$$

Solving this system over the entire atmospheric grid has been shown to be the major bottleneck in atmospheric simulations $[18,19,51,55]$. Before discussing the particulars of these kinetics equations, the standard numerical techniques for the general ODE problem must be examined.

\subsection{Methods for the General ODE Problem}

Numerical techniques for the general ODE problem are, like their PDE counterparts, produced by replacing derivatives with finite differences. Alternatively, one can apply standard numerical quadrature techniques to interpolating functions fit to the discrete values of the dependent variable $[25,37,58]$. This results in the rather large class of linear multistep methods $[12,28]$. In a linear multistep method, the discrete values are defined by the formula: .

$$
y^{n}=\sum_{i=1}^{K_{1}} \alpha_{i} y^{n-i}+\Delta t \sum_{i=0}^{K_{2}} \beta_{i} y^{n-i}
$$


where the $\alpha_{i}, \beta_{i}, K_{1}$, and $K_{2}$ are constants for a particular method and $\dot{y}^{n-i}=R\left(y^{n-i}, t_{n-i}\right)$ (cf. Eq. (7)) [12]. The value $K=\max \left(K_{1}, K_{2}\right)$ is the number of past values involved, so that Eq. (8) is said to be a K-step method. The methods can also be written in terms of a variable stepsize $[9,11,12]$.

The simplest examples are the forward Euler method

$$
y^{n}=y^{n-1}+\Delta t y^{n-1}
$$

and the backward Euler method

$$
y^{n}=y^{n-1}+\Delta t \hat{y}^{n} .
$$

The most important distinction between these methods is whether a given method is explicit or implicit. The methods described by Eq. (8) are explicit if $\beta_{0}=0$ and implicit otherwise. For an implicit method, a nonlinear algebraic system must be solved at each timestep which can be a computationally costly procedure. Common methods for treating these algebraic systems are discussed later.

Another distinction is the particular order of a method. The order is the largest integer $p$ for which

$$
y\left(t_{n}\right)-\sum_{i=1}^{K_{1}} \alpha_{i} y^{n-i}-\Delta t \sum_{i=0}^{K_{2}} \beta_{i} y^{n-i}=O\left(\Delta t^{p+1}\right)
$$

as $\Delta t \rightarrow 0$. If $y$ is smooth, it is equivalent to say that the local truncation error satisfies $y^{n}-y\left(t_{n}\right)=O\left(\Delta t^{p+1}\right)[12]$.

Common subgroups of methods, belonging to the class of linear multistep methods, are the Adams methods and the backward differentiation formulas (BDFs). The Adams methods [37], popular in solving non-stiff problems, are obtained by having only the term $y^{n-1}$ in the first sum in Eq. (8). The BDFs [12, 24, 25], most common for stiff problems, are 
obtained by setting $K_{2}=0$ in Eq. (8). More detail is given to these methods after an examination of the phenomenon of stiffness in ODE systems.

\subsection{Stiff Systems of Ordinary Differential Equations}

Put simply, stiffness arises when components of the ODE system have widely differing timescales or lifetimes. This situation causes the problem to be particularly challenging from a numerical standpoint, and many specialized techniques have been developed for treating these problems, both for the general stiff problem [10,12, 17, 24, 25, 37, 49, 53] and for chemical kinetics problems $[3,4,19,27,54,59,61,65,66,76]$. The difficulty is that when a standard explicit method is used, the timestep is restricted to the order of the shortest lifetime in the problem even after such transient terms have died out. This restriction on the timestep is necessary so that the method does not become unstable. A method is said to be unstable when any roundoff error that gets introduced into the calculation is successively magnified until it comes to dominate the true solution. As long as machines compute in finite precision, the methods carried out on them must be stable. Stiffness was first identified as an important property of the ODEs describing chemical kinetics by Curtis and Hirschfelder [17].

There are several approaches that can (and have been) used to alleviate the problem of stiffness in atmospheric chemistry problems. One possibility is to assume a steady state for all of the faster species (i.e. those with short timescales). This is done to some extent in all models and the loss in accuracy in doing so is open to debate. This study attempts to avoid this approximation if at all possible, since this provides the most mathematically rigorous model.

Another approach is the so-called family or lumping approach $[5,7,16,23,63]$. Here, chemical species are combined or lumped together into chemical families (such as $N O_{x}$ or $O_{x}$ etc.) again in an attempt to eliminate the faster moving transients. This study uses no 
lumping of any kind, since, as with the steady state approximation, it is not the most rigorous way to model and is subject to potentially significant error.

The final approach to stiff problems is to use numerical techniques to overcome their specific difficulties. Doing so requires a more in-depth examination of the concept of stiffness.

\subsection{Basic Definition}

Stiffness occurs in a physical problem when widely varying timescales are simulated. Mathematically, this situation manifests itself with the existence of widely differing magnitudes of the eigenvalues of the ODE system modeling the physical problem. This situation can be illustrated by a simple linear initial value problem and serves to motivate the definition of a stiff problem. The following example is taken from Lambert [37].

Consider the linear initial value problem with constant coefficients:

$$
\dot{y}=A y
$$

and let

$$
A=\left[\begin{array}{ccc}
-21 & 19 & -20 \\
19 & -21 & 20 \\
40 & -40 & -40
\end{array}\right]
$$

with

$$
y(0)=\left[\begin{array}{c}
1 \\
0 \\
-1
\end{array}\right]
$$

Now, the analytic solution to this problem is given by 


$$
y(t)=\left[\begin{array}{c}
\frac{1}{2} e^{-2 t}+\frac{1}{2} e^{-40 t}(\cos 40 t+\sin 40 t) \\
\frac{1}{2} e^{-2 t}-\frac{1}{2} e^{-40 t}(\cos 40 t+\sin 40 t) \\
-e^{-40 t}(\cos 40 t+\sin 40 t)
\end{array}\right]=\left[\begin{array}{c}
u(t) \\
v(t) \\
w(t)
\end{array}\right]
$$

and is shown in figure 1. The important point to note here is that for time values greater than $0.1, u$ and $v$ are nearly indistinguishable, and vary slowly with time, while $w$, the last component, is essentially zero.

If the values obtained from the analytic result are used to produce an initial condition for $t=0.1$, and the forward Euler method (with a stepsize of 0.04 ) is used for an integration, the computation diverges due to instability. This occurs even though the solution curves are all gently varying in the region of integration. The use of an implicit method, such as the backward Euler method, would eliminate this restriction on step length and will be discussed later. The trade-off in the use of such a technique is that a matrix must be inverted at each timestep. Even though a matrix invert is easily accomplished for a 3 component problem such as this one, the work required on larger problems would be significant. Explicit schemes, such as the forward Euler method discussed here, are computationally cheap to implement and very efficient on vector and parallel machines. For the moment, therefore, this study will focus on the problems that these explicit schemes suffer when used on stiff problems.

Now consider a different matrix:

$$
A=\left[\begin{array}{ccc}
-2 & 0 & 0 \\
0 & -2 & 0 \\
0 & 0 & 0
\end{array}\right]
$$

with the initial value as: 
FIGURE 1. Solution plot for the first example stiff problem.

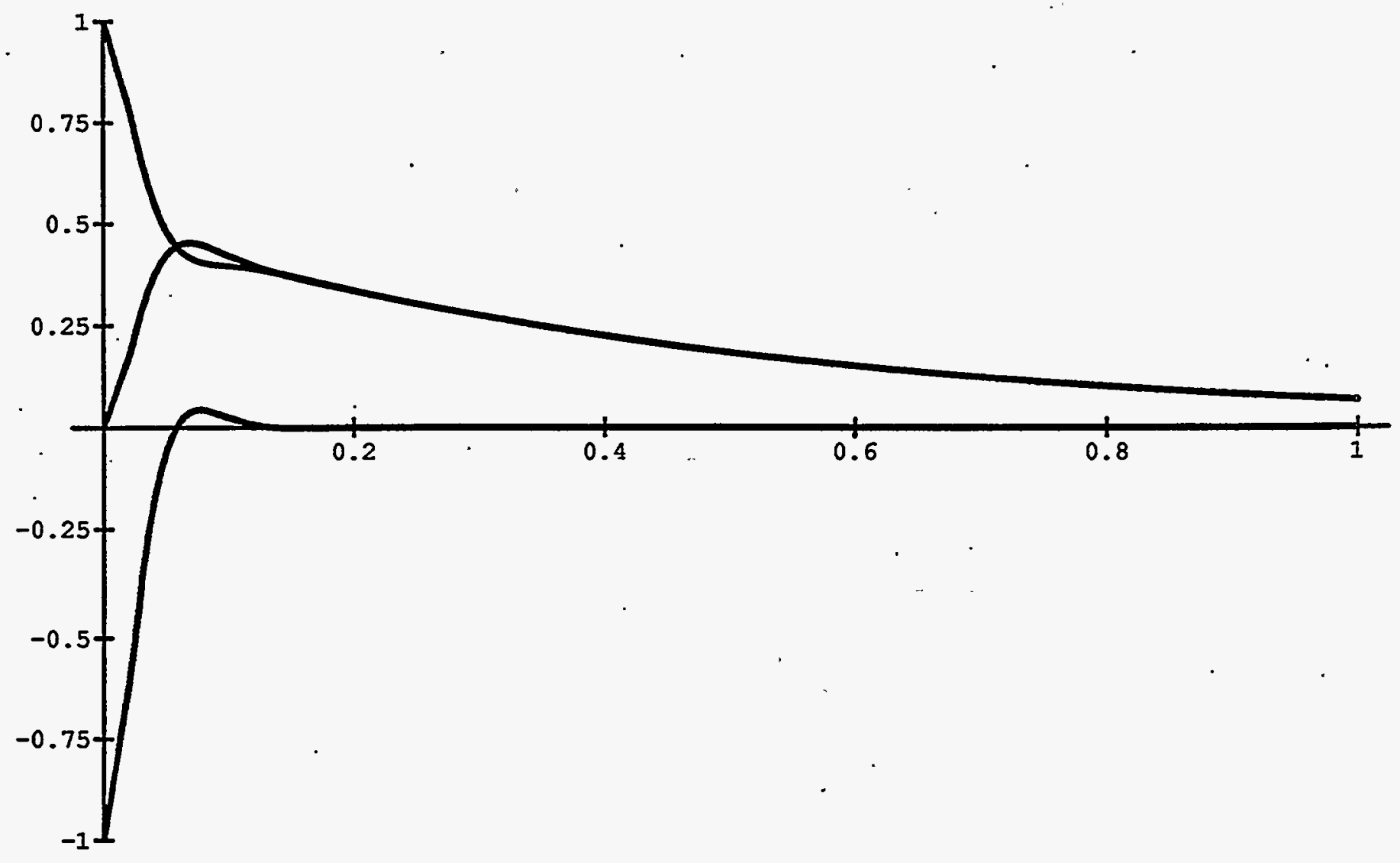




$$
y(0.1)=\left[\begin{array}{c}
\frac{1}{2} e^{-0.2} \\
\frac{1}{2} e^{-0.2} \\
0
\end{array}\right]
$$

which has the analytic solution

$$
y(t)=\left[\begin{array}{c}
\frac{1}{2} e^{-2 t} \\
\frac{1}{2} e^{-2 t} \\
0
\end{array}\right]
$$

(This second problem is designed to have an analytic solution that is almost identical to the first example in the range $t \geq 0.1$ ). The application of the forward Euler technique, using the same stepsize, somewhat surprisingly yields a stable computation! This is surprising since the two examples were nearly identical on the region of integration.

To understand this situation, consider the more general first order ODE:

$$
\dot{y}=f(y, t)
$$

To guarantee that a computation be stable, most explicit methods (including the forward Euler method) place a bound on the product $h \lambda_{i}$, where the $\lambda_{i}$ are the eigenvalues of the Jacobian $\frac{\partial f}{\partial y}$, and $h$ is the stepsize used [37]. For the simple linear constant coefficient example, $\frac{\partial f}{\partial y}=A$.

Now, consider the eigenvalues of the two sample matrices examined: the eigenvalues of the first matrix are $-2,-40 \pm 40 i$, while the eigenvalues of the second are $-2,-2,0$. The stability requirement for the forward Euler method (to be mentioned in a little more detail later on) imposes a max stepsize of $h=0.025$ for the first matrix and $h=1.0$ for the 
second matrix. This is true even though, as emphasized earlier, their analytic solution curves are indistinguishable on the region of integration. The eigenvalues responsible for this behavior are $-40 \pm 40 i$, the very eigenvalues whose contribution to the analytic solution is most negligible. This, then, is the phenomenon of stiffness: the existence of these (relatively) fast moving transients that contribute little to the solution curve, but whose numerical remnants linger to destroy the stability of the computation. This motivates the following "definition".

\subsubsection{Definition}

The linear constant coefficient problem $\dot{y}=A y$ is said to be stiff if:

1. $\operatorname{Re}\left(\lambda_{i}\right)<0, \forall i$

2. $\operatorname{Max}\left(\operatorname{Re}\left(\lambda_{i}\right)\right) \gg \operatorname{Min}\left(\operatorname{Re}\left(\lambda_{i}\right)\right)$

where, as before, the $\lambda_{i}$ are the eigenvalues of $A$. Note that this is not the most precise of definitions. Any system satisfying condition one will exhibit some degree of stiffness. One can define the quantity

$$
\frac{\operatorname{Min}\left(\operatorname{Re}\left(\lambda_{i}\right)\right)}{\operatorname{Max}\left(\operatorname{Re}\left(\lambda_{i}\right)\right)}
$$

called the stiffness ratio, as a measure of how stiff the system is. The stiffness ratio in the first example matrix was 20 . This turns out to be quite modest, as stiffness ratios of $10^{6}$ or more are not uncommon in atmospheric chemical kinetics $[12,37,65]$.

For the more general first order IVP (Eq. (18)), stiffness is seen if the eigenvalues of the Jacobian behave in a similar fashion. In this case, the eigenvalues are no longer constant, so it is said that the problem is stiff in an interval $I$ if the eigenvalues meet the criteria in the definition for all $t \in I$. 


\subsection{Stability for Stiff Systems}

The goal in treating stiff problems is to find numerical integration techniques whose regions of absolute stability are "large enough". The first step is to take a closer look at the stability requirement of the forward Euler technique.

For absolute stability, forward Euler requires that [37]

$$
\left|1+h \lambda_{i}\right|<1
$$

where $h$ is the stepsize and $\lambda_{i}$ is any eigenvalue of the Jacobian $\frac{\partial f}{\partial y}$ of the right hand side of the IVP (18). This relation must be satisfied by all of the eigenvalues. In words, the relation says that the product $h \lambda_{i}$ must lie within the circle of radius 1 about -1 in the complex plane (this circle is often referred to as the region of absolute stability). Hence, stiff systems, having eigenvalues with relatively large negative real parts, require a correspondingly small stepsize to meet the stability requirement. Higher order methods have larger regions, but can still run into the same types of difficulties. What is desired is a method whose region of absolute stability is in some sense infinite. The following definition is due to Dahlquist [20].

\subsubsection{Definition}

A numerical method is said to be A-stable if its region of absolute stability includes the entire left hand half plane $\operatorname{Re}\left(h \lambda_{i}\right)<0$. Note that when an A-stable method is used to treat a stiff problem, stepsize is not constrained by stability!

Consider the backward Euler method. The stability criterion here is [37]

$$
\left|1-h \lambda_{i}\right|^{-1}<1
$$

and is satisfied for any stepsize given a stiff problem. The backward Euler method is therefore A-stable. Figure 2 illustrates the forward Euler region of absolute stability, while figure 3 illustrates A-stability. The following theorem is regarding A-stability: 
FIGURE 2. Forward Euler Region of Absolute Stability

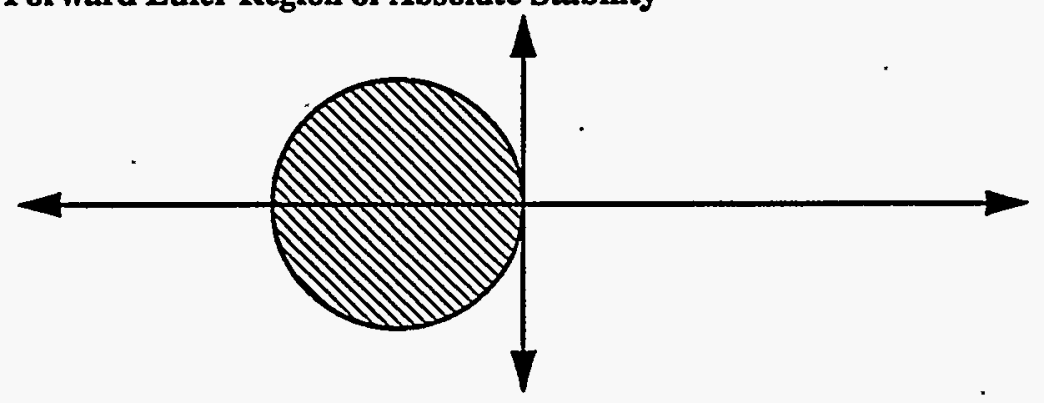

FIGURE 3. A-Stability

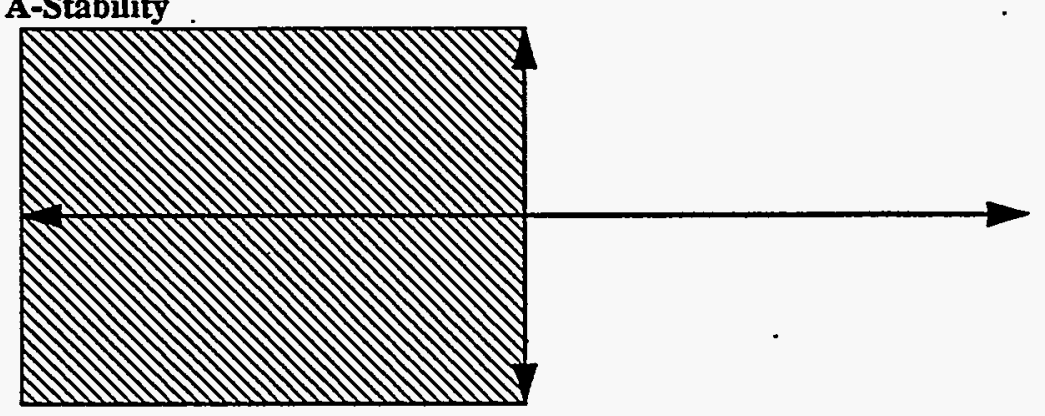

\subsubsection{Theorem (regarding A-stability)}

1. An explicit linear multistep method cannot be A-stable

2. The order of an A-stable implicit linear multistep method cannot exceed two.

3. The second order implicit linear multistep method with the smallest error constant is the trapezoidal rule.

A proof is given by Dahlquist [20].

A-stability seems to be a pretty demanding restriction! It is limited to second order methods. The restriction can be weakened with a concept due to Widlund [71]:

\subsubsection{Definition}

A numerical method is said to be $A(\alpha)$-stable, $\alpha \in\left(0, \frac{\pi}{2}\right)$, if its region of absolute stability includes the infinite wedge

$$
W_{\alpha}=\left\{h \lambda_{i} \mid\left(-\alpha<\pi-\arg \left(h \lambda_{i}\right)<\alpha\right)\right\}
$$

It is said to be A(0)-Stable if it is stable for some sufficiently small $\alpha$. Note that for a given problem, $h \lambda_{i}$ will lie either inside or outside the wedge for all values of $h$. 


\subsubsection{Theorem}

1. An explicit linear multistep method cannot be $A(0)$-Stable.

2. There is only one $A(0)$-Stable linear $k$-step method whose order exceeds $k+1$, namely the trapezoidal rule.

3. For each $\alpha \in\left[0, \frac{\pi}{2}\right)$ there exist $A(\alpha)$-Stable linear $k$-step methods of order $p$ for $k=p=3$ and $k=p=4$.

This result is given by Widlund [71]. So a slight weakening of A-Stability allows up to fourth order methods, while $A(0)$-stability allows methods of arbitrary order. $A(\alpha)$-stability is illustrated in Figure 4.

FIGURE 4. $A(\alpha)$-Stability

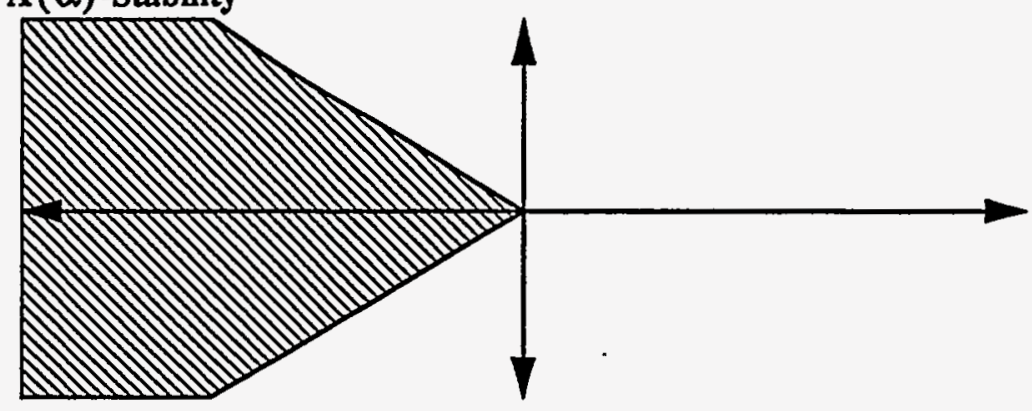

\subsection{The Problem With Implicitness}

The theorems of the preceding section illustrate that A-Stable and A(0)-stable imply implicit techniques. Thus, at each time step, a (potentially nonlinear) system of simultaneous equations must be solved (cf. Eq. (8)):

$$
y_{n+k}=h \beta_{k} f\left(t_{n+k} y_{n+k}\right)+g
$$

where $g$ is some known vector.

One scheme: Use a predictor-corrector type [37] method. Problem: Correcting to convergence requires that $L\left|h \beta_{k}\right|<1$, where $L$ is the Lipschitz constant for $f(t, y)$. Now, the Lipschitz constant may be taken as $\left\|\frac{\partial f}{\partial y}\right\|$, and since the matrix norm satisfies $\|A\| \geq \rho(A)=\max \left(\left|\lambda_{i}\right|\right)$, it is clear that stiff systems imply LARGE Lipschitz con- 
stants. Thus, the convergence requirement is usually as bad as the explicit stability requirement.

The acceptable alternative to predictor-corrector is to use a Newton type $[12,24,25,37]$ iteration. That is, to solve for $F(x)=0$, fix an initial guess and then compute the iterates:

$$
y^{s+1}=y^{s}-\left[J\left(y^{s}\right)\right]^{-1} F\left(y^{s}\right)
$$

Applied to the linear multistep formula (Eq. (23)), this gives:

$$
y_{n+k}^{s+1}=y_{n+k}^{s}-\left[I-h \beta_{k} \frac{\partial f\left(t_{n+k} y_{n+k}^{s}\right)}{\partial y}\right]^{-1}\left[y_{n+k}^{s}-h \beta_{k} f\left(t_{n+k} y_{n+k}^{s}\right)-g\right]
$$

Now, the question of convergence can be quite complicated [37], but is not nearly as bad a restriction on stepsize as explicit stability bounds provided the initial guess is "close enough". That is where a good predictor comes in handy.

So, at each time step it is necessary to (possibly more than once):

- Evaluate the Jacobian matrix

- Invert the Jacobian matrix

Does the increase in stepsize make up for the additional computation that must be done? In fact, the extra computation that must be performed by an implicit method is always outweighed by the stability advantage [35]. One possibility for speeding the process up is to hold the Jacobian constant during the Newton iteration or even for several time steps and see if the iterates still converge.

\subsection{LSODE}

The LSODE [46] package is used for comparison in this study. LSODE is state-of-the-art in the robust implementation of methods for the general ODE problem. For the treatment of stiff problems, LSODE uses the backward differentiation formulas (BDFs) [25] men- 
tioned in section 1.2. These methods are the most popular currently used for the general stiff problem [52]. The BDFs possess stiff stability [25] and do not suffer the stepsize constraint that the traditional explicit methods do. Thus, the numerical stepsize is constrained only by the user's accuracy requirement.

LSODE also includes the implicit Adams methods (mentioned in section 2.1) which are well suited for nonstiff problems. As with the BDFs, these methods are members of the family of linear multistep methods (Eq. (8)). LSODE implements these methods in a way that allows both the stepsize and the method order to dynamically vary throughout the course of the integration. The method orders vary from 1 to 12 for the Adams methods and from 1 to 5 for the BDFs. The ability to dynamically vary the method order and the stepsize is critical to the efficient use of the multistep methods [46]. This allows them to "tiptoe" through phases of the problem where solution components are rapidly changing, while integrating slowly changing phases by leaps and bounds.

LSODE consists of 21 subprograms and a BLOCK DATA module. The package has been designed so that the user need only communicate with a single subprogram, called LSODE for convenience. LSODE's features include a highly flexible user interface, highly modularized code, dynamic storage allocation, a wide range of linear algebra modules, and a wide range of error types. It has been designed to virtually eliminate the need for user adjustments to the code before it can be used effectively. The different capabilities of the code can be exploited by setting flags in the parameters passed to the program. This philosophy eliminates the need for the user to become familiar with the inner workings of the code, allowing it to be used almost as a black box and reducing the possibility of errors being introduced into the code by user modification.

In this study, LSODE will be called to use the BDFs, using a Newton iteration to solve the resulting nonlinear system. The Jacobian matrix is automatically generated using finite 
differences and is inverted as often as the code deems necessary. This is one of the code's optimizations, since it is sometimes unnecessary to change the Jacobian at every step.

\subsection{Basic Language of Chemical Kinetics}

This work concerns the treatment of the chemical kinetic portion of the chemical mass continuity equation (Eq. (1)), so a brief digression into the language of kinetics is in order.

Chemical kinetics is the study of the rates of reactions. These methods were developed for the resolution and understanding of the systems encountered by chemists. The rate or velocity, $v$, of a reaction, tells how quickly it is occurring. If $c$ is the chemical concentration, then:

$$
v=\frac{d c}{d t}
$$

which gives the rate of the reaction.

The Rate Law [57] states that $v$ is a function of the concentrations of the species involved.

$$
v=f(\text { concentrations) }
$$

Substances that influence the velocity of a reaction can be grouped into two categories:

1. Those whose concentrations change with time (Reactants, Products, Intermediates)

2. Those whose concentrations do not change with time (Catalysts, Steady State Intermediates)

The stoichiometry of a process shows how many moles of each reactant are needed to form each mole of products. For Example:

$$
2 \mathrm{H}_{2}+\mathrm{O}_{2} \rightarrow 2 \mathrm{H}_{2} \mathrm{O}
$$

The mechanism details how the molecules react to form products. The mechanism is, in general, a set of elementary reactions consistent with the stoichiometric relation. The pre- 
vious stoichiometry, for example, in the gas phase, is thought to be a chain involving hydrogen, oxygen and hydroxyl radicals:

$$
\begin{aligned}
\mathrm{H}_{2} & \rightarrow 2 \mathrm{H} \\
\mathrm{H}+\mathrm{O}_{2} & \rightarrow \mathrm{OH}+\mathrm{O} \\
\mathrm{OH}+\mathrm{H}_{2} & \rightarrow \mathrm{H}_{2} \mathrm{O}+\mathrm{H} \\
\mathrm{O}+\mathrm{H}_{2} & \rightarrow \mathrm{OH}+\mathrm{H}
\end{aligned}
$$

Each reaction is an elementary one; the four taken together describe the proposed mechanism.

The kinetic order of a reaction describes the way in which the velocity depends upon the concentration. Consider a reaction whose stoichiometry is

$$
A+B \rightarrow P
$$

For many such reactions, the rate law is of the form [57]

$$
\frac{d c}{d t}=k c_{A}^{m} c_{B}^{n} c_{P}^{q}
$$

where the concentrations are raised to the powers $m, n$, and $q$, that are usually (but not always) integers or zero. In this study, they will always be zero or one.

The order of a reaction with respect to a particular component is just the exponent of the concentration term. The overall order is the sum of the exponents of all components. The constant $k$ is the rate coefficient of the reaction.

If the concentration of a particular component doesn't change during the reaction, it is frequently omitted from the rate law expression.

Note: One cannot deduce the rate law from the stoichiometry of a reaction. It must be measured by experiment. 


\subsection{Chemical Kinetics Equations}

This study is primarily concerned with the chemical kinetics portion of the atmospheric advection-diffusion-reaction equations (Eq. (7)). This is split from the advective-diffusive part and results in a nonlinear system of ordinary differential equations having the form: $[4,57,65,66]$

$$
\frac{d y}{d t}=R(y, t)=P(y, t)-\hat{L}(y, t) y-\bar{L}(y, t) y^{2}
$$

where $y(t)$ is the vector of chemical concentrations, $y^{2}(t)$ is the vector containing the square of each chemical concentration, $P(y, t)$ is a vector representing the "production rate" term, while $\hat{L}(y, t)$ and $\bar{L}(y, t)$ are diagonal matrices representing the "loss rate" terms.

Interesting to note are the forms of the production and loss rates. In terms of section 3.0, $P_{i}=\sum_{q} k_{q} y_{u_{q}}^{j_{q}} y_{v_{q}}^{r_{q}} y_{w_{q}}^{s_{q}}$, where the components of the chemical concentration vector are raised to the powers $j_{q}, r_{q}, s_{q}$ which are either zero or one, depending on the chemical reactions in the problem. That is to say, this production rate term is the sum of all of the terms from the rate law for each reaction producing species $i$. These terms are of integral order of at most one in their dependence on other species. Further, $u_{q}, v_{q}, w_{q} \neq i$ for each $q$, so that $P_{i}$ does not depend on $y_{i}$. The $k_{q}$ represent reaction rate coefficients (as in Eq. (34)) and may be time dependent (for photochemical reactions, which are diumally varying). Finally, $\hat{L}_{i i}$ and $\bar{L}_{i i}$ which are henceforth denoted as $\hat{L}_{i}$ and $\bar{L}_{i}$, have the forms $\hat{L}_{i}=\sum_{q} k_{q} y_{u_{q}}^{j_{q}} y_{v_{q}}^{r_{q}}$ and $\bar{L}_{i}=\sum_{q} k_{q} y_{u_{q}}^{j_{q}}$ where the exponents of the chemical concentrations are.again either zero or one and the entire term is independent of $y_{i}$ [57]. These are the corresponding rate law terms for reactions destroying species $i$.

\subsection{The Chapman Atmosphere}

Consider the equations describing the generation and destruction of ozone in the simple . Chapman model. The Chapman model was the first attempt (in the late 1930s) to explain 
the ozone distribution in the stratosphere. The Chapman mechanism assumes that only oxygen-containing chemistry is sufficient to explain ozone distribution. In this case, the atmosphere includes only the chemistry of $\mathrm{O}_{2}, \mathrm{O}$ and $\mathrm{O}_{3}[12,22,36$. In this situation there are four chemical reactions:

\begin{tabular}{lll|l}
\hline \hline$O+O_{2}+M$ & $\rightarrow$ & $O_{3}+M$ & $k_{1}$ \\
$O+O_{3}$ & $\rightarrow$ & $2 O_{2}$ & $k_{2}$ \\
$O_{2}+h v$ & $\rightarrow$ & $2 O$ & $k_{3}$ \\
$O_{3}+h v$ & $\rightarrow$ & $O+O_{2}$ & $k_{4}$ \\
\hline
\end{tabular}

where the $k_{i}$ denote the reaction rate coefficients, $M$ denotes an extra molecule required to carry off excess energy, and $h v$ denotes the absorption of light (a photochemical reaction). Ozone formation occurs through the first reaction while ozone destruction occurs in the second and last reactions. For this problem, the concentration of molecular oxygen, $\mathrm{O}_{2}$, is held constant (making the reasonable assumption that the loss of $O_{2}$ from this chemistry is negligible). The first two reaction rates are fixed (functions of temperature only) while the latter two (being driven by the absorption of light) will vary diurnally.

Set $y_{1}, y_{2}, y_{3}$ to be the concentrations of $O, O_{3}, O_{2}$, respectively. Again, the concentration of $\mathrm{O}_{2}$ is assumed to be unchanging. The resulting system of kinetic equations takes the form of Eq. (42), for $y=\left[\begin{array}{l}y_{1} \\ y_{2}\end{array}\right]$, with

$$
\begin{gathered}
P(y, t)=\left[\begin{array}{c}
2 k_{3}(t) y_{3}+k_{4}(t) y_{2} \\
k_{1} y_{1} y_{3}
\end{array}\right] \\
\hat{L}(y, t)=\left[\begin{array}{cc}
k_{1} y_{3}+k_{2} y_{2} & 0 \\
0 & k_{2} y_{1}+k_{4}(t)
\end{array}\right] \\
(\bar{L}=0) .
\end{gathered}
$$


The constant values are:

$$
\begin{aligned}
& y_{3}=3.7 \times 10^{16} \\
& k_{1}=1.63 \times 10^{-16} \\
& k_{2}=4.66 \times 10^{-16}
\end{aligned}
$$

and

$$
k_{i}=\left\{\begin{array}{c}
\exp \left[\frac{-a_{i}}{\sin \omega t}\right], \sin \omega t>0, i=3,4 \\
0, \sin \omega t \leq 0
\end{array} .\right.
$$

with

$$
a_{3}=22.62, a_{4}=7.601, \omega=\frac{\pi}{43200} \text {. }
$$

The constant 43200 is just twelve hours in seconds, so that the diurnally varying rate coefficients $\left(k_{3}\right.$ and $\left.k_{4}\right)$ have 24 hour periods. Initial conditions assumed are $y_{1}(0)=10^{6}, y_{2}(0)=10^{12}$.

The Chapman mechanism has important characteristics shared by the larger problems in atmospheric chemistry [12] in that:

- The Jacobian of the differential system is non-constant.

- The diurnal effect is present.

- The problem is sufficiently stiff

The Chapman problem will be the initial testbed for the development of the basic ideas for the numerical techniques in the next chapter. 
CHAPTER 2

\section{Preconditioned Time Differencing: Development and Initial Results for Atmospheric Chemistry}

\subsection{Preconditioned Time Differencing}

This chapter develops the methods for treating stiff diurnal kinetics equations from atmospheric chemistry. The goal is to get the "best of both worlds", by developing an explicit class of method that is unconditionally (or at least-nearly unconditionally) stable. Consider the first order nonlinear system of ordinary differential equations:

$$
\frac{d y}{d t}=f(y, t), \quad y(0)=y_{0}
$$

Eq. (1) is assumed to be stiff. Stability constraints brought about by the stiffness of the problem generally force the use of a $k$-step implicit multistep formula $[25,37]$

$$
y^{n+1}=\Delta t \beta_{k} f\left(y^{n+1}, t_{n+1}\right)+g^{n}
$$

(with $\Delta t=$ stepsize, $t_{n+1}=(n+1) \Delta t, y^{n+1} \approx y\left(t_{n+1}\right), \beta_{k}=$ const., and $g^{n}$ a known vector) for its solution. In this chapter, the first order backward Euler method

$$
y^{n+1}=y^{n}+\Delta t f^{n+1}
$$

(where $f^{n+1}=f\left(y^{n+1}, t_{n+1}\right)$ ) and the second order trapezoid rule 


$$
y^{n+1}=\frac{\Delta t}{2}\left(f^{n+1}+f^{n}\right)+y^{n}
$$

are considered.

The major task in implicit schemes is the solution of the nonlinear system of equations (Eq. (2)) at each time step. Many ODE packages, such as the ODEPACK routine LSODE $[28,46]$ and the DAE solver DASSL $[8,44]$, resort to a Newton iteration for solving this system. Although the Newton iteration converges rapidly, it requires the computationally cumbersome construction, storage, and inversion of a large (and often non-sparse) Jacobian matrix [21]. This problem can be avoided by considering the fixed point iterative method as a means of solution.

Fixed point iteration (or Picard iteration) for the solution of the nonlinear system in Eq. (2) begins by defining the function

$$
G(y)=\Delta t \beta_{k} f\left(y, t_{n+1}\right)+g^{n}
$$

and then performing the iteration

$$
\begin{gathered}
\left(y^{n+1}\right)^{(s+1)}=G\left(\left(y^{n+1}\right)^{(s)}\right) \\
\left(y^{n+1}\right)^{(0)}=\text { arbitrary. }
\end{gathered}
$$

Convergence of the iteration to $y^{n+1}$ will occur if the initial guess $\left(y^{n+1}\right)^{(0)}$ is chosen in a neighborhood of contraction of $G$ about $y^{n+1}$ [21]. If $G$ is not contractive at $y^{n+1}$ then the fixed point iteration may diverge. A sufficient condition for the existence of a contractive neighborhood is that the spectral radius of the Jacobian of $G$ at $y^{n+1}$ be less than unity. If such a neighborhood does exist, then one must assure that the initial iterate $\left(y^{n+1}\right)^{(0)}$ is a member of this contractive neighborhood. 
The problem of diverging or slowly converging fixed point iteration schemes can often be rectified by "preconditioning" $[26,47]$. That is, a function $F(u, v, t)$ is defined such that $F(v, v, t)=G(v, t) ;$ the iterates are then computed in the following fashion:

$$
\begin{gathered}
\left(y^{n+1}\right)^{(s+1)}=F\left(\left(y^{n+1}\right)^{(s+1)},\left(y^{n+1}\right)^{(s)}, t_{n+1}\right) \\
\cdot\left(y^{n+1}\right)^{(0)}=\text { arbitrary. }
\end{gathered}
$$

If this procedure were to converge to $y^{n+1}$, then

$$
y^{n+1}=F\left(y^{n+1}, y^{n+1}, t_{n+1}\right)=G\left(y^{n+1}, t_{n+1}\right)=\Delta t \beta_{k} f\left(y^{n+1}, t_{n+1}\right)+g^{n}
$$

and the desired solution is obtained. Although each iteration requires the construction and solution of another nonlinear system of equations, in the case of chemical kinetics equations a preconditioner $F$ may be defined that is easily solved.

The preconditioners that will be considered in this chapter are the Jacobi preconditioner

$$
F_{i}(u, v, t)=\Delta t \quad \beta_{k} f_{i}\left(v_{1}, v_{2}, \ldots, v_{i-1}, u_{i}, v_{i+1}, \ldots, v_{N}, t\right)+g_{i}^{n},
$$

and the Gauss-Seidel preconditioner

$$
F_{i}(u, v, t)=\Delta t \beta_{k} f_{i}\left(u_{1}, u_{2}, \ldots, u_{i-1}, u_{i}, v_{i+1}, \ldots, v_{N}, t\right)+g_{i}{ }^{n} .
$$

Here, $N$ is the number of dependent variables in the system Eq. (1).

Now, if the iteration is terminated prematurely after $l$ iterations $\left(y^{n+1}=\left(y^{n+1}\right)^{(l)}\right)$ then time differencing methods are generated in the form:

\section{Algorithm 1.1}

1. Set $\left(y^{n+1}\right)^{(0)}$ (the initial guess).

2. For $s=1,2, \ldots, l$, compute $\left(y^{n+1}\right)^{(s)}=F\left(\left(y^{n+1}\right)^{(s)},\left(y^{n+1}\right)^{(s-1)}, t_{n+1}\right)$

3. Set $y^{n+1}=\left(y^{n+1}\right)^{(l)}$ 
These methods are called Preconditioned Time Differencing Methods $[3,4,47]$. It should be noted that the idea of premature termination of Newton's iteration has been used successfully in other contexts (see [1] for example), but in these situations a Jacobian matrix needs to be formed and inverted. In addition to the choice of preconditioner, there is also the issue of choosing the initial iterate, or initial guess in step 1 of algorithm 1.1. Commonly used is the so-called "identity predictor" $[3,4]$ :

$$
\left(y^{n+1}\right)^{(0)}=y^{n}
$$

The idea behind preconditioned time differencing is illustrated by considering the application of the Jacobi preconditioner (Eq. (5)) to the backward Euler method (Eq. (3)) with the identity predictor (Eq. (7)) as the initial guess. Taking only a single iteration $(l=1$ in algorithm 1.1) yields, for $i=1 \ldots . . N$ :

$$
y_{i}^{n+1}=\Delta t f_{i}\left(y_{1}^{n}, \ldots, y^{n}{ }_{i-1}, y_{i}^{n+1}, y^{n}{ }_{i+1}, \ldots, t_{n+1}\right)+y^{n}{ }_{i}
$$

A Gauss-Seidel preconditioner (Eq. (6)) yields in a similar manner:

$$
y_{i}^{n+1}=\Delta t f_{i}\left(y_{1}^{n+1}, \ldots, y^{n+1}{ }_{i-1}, y_{i}^{n+1}, y^{n}{ }_{i+1}, \ldots, t_{n+1}\right)+y^{n}{ }_{i}
$$

For an arbitrary linear multistep method Eq. (2) and an arbitrary number of iterations, the Jacobi and Gauss-Seidel preconditioners take the respective forms:

$$
\begin{gathered}
\left(y_{i}^{n+1}\right)^{s+1}=\Delta t \beta_{k} f_{i}\left(\left(y_{1}^{n+1}\right)^{s}, \ldots,\left(y_{i}^{n+1}\right)^{s+1}, \ldots,\left(y_{N}^{n+1}\right)^{s}, t_{n+1}\right)+g^{n} \\
\left(y_{i}^{n+1}\right)^{s+1}=\Delta t \beta_{k} f_{i}\left(\left(y_{1}^{n+1}\right)^{s+1}, \ldots,\left(y_{i}^{n+1}\right)^{s+1},\left(y^{n+1}{ }_{i+1}\right)^{s}, \ldots, t_{n+1}\right)+g^{n}
\end{gathered}
$$

For this specific class of problem, preconditioned time differencing methods will yield explicit schemes with expanded regions of absolute stability. 


\subsection{Chemical Kinetics Equations}

The preconditioned time difference methods are applied to the Chapman problem described at the end of chapter 1 (section 4.1 ). Their behavior is analyzed on a linearized system of equations derived from the Chapman problem. The equations describing the nonlinear ODE system are restated here, for completeness:

$$
\begin{gathered}
y=\left[\begin{array}{l}
y_{1} \\
y_{2}
\end{array}\right] \\
\frac{d y}{d t}=R(y, t)=P(y, t)-\hat{L}(y, t) y-\bar{L}(y, t) y^{2} \\
P(y, t)=\left[\begin{array}{c}
2 k_{3}(t) y_{3}+k_{4}(t) y_{2} \\
k_{1} y_{1} y_{3}
\end{array}\right] \\
\hat{L}(y, t)=\left[\begin{array}{cc}
k_{1} y_{3}+k_{2} y_{2} & 0 \\
0 & k_{2} y_{1}+k_{4}(t)
\end{array}\right] \\
(\bar{L}=0) .
\end{gathered}
$$

The values of all numerical constants may be found in section 4.1 of chapter 1 . The diurnally varying rate coefficients are mathematically idealized, and take the form

$$
k_{i}=\left\{\begin{array}{c}
\exp \left[\frac{-a_{i}}{\sin \omega t}\right], \sin \omega t>0 \\
0, \sin \omega t \leq 0
\end{array}, i=3,4\right.
$$

\subsubsection{Linearized Chapman Mechanism}

For the purpose of analysis, the behavior of the preconditioned time differencing schemes for solving the nonlinear Chapman mechanism is studied by analyzing their behavior on the linearized system of equations

$$
\frac{d y}{d t}=A \mathrm{y}
$$


where

$$
\begin{gathered}
A=D R\left(y_{0}, t\right) \\
=\left[\begin{array}{ll}
\frac{\partial R_{1}}{\partial y_{1}} & \frac{\partial R_{1}}{\partial y_{2}} \\
\frac{\partial R_{2}}{\partial y_{1}} & \frac{\partial R_{2}}{\partial y_{2}}
\end{array}\right] \\
=\left[\begin{array}{cc}
\left(-k_{1} y_{3}-k_{2} y_{2}\right) & \left(-k_{2} y_{1}+k_{4}\right) \\
\left(k_{1} y_{3}-k_{2} y_{2}\right) & \left(-k_{2} y_{1}-k_{4}\right)
\end{array}\right]
\end{gathered}
$$

Note that

$$
A \approx\left[\begin{array}{cc}
-a & -\varepsilon \\
a & -\varepsilon
\end{array}\right]
$$

with $a=6.03, \varepsilon=4.66 \times 10^{-10}$.

In this case, $k$-step implicit numerical schemes take the form [47]

$$
y^{n+1}=\sum_{j=-1}^{k-1} Q_{j}(\Delta t A) y^{n-j}
$$

where the $Q_{j}$ 's are real polynomials, and at each time-step, the linear system

$$
\left[I-Q_{-1}(\Delta t A)\right] y^{n+1}=\sum_{j=0}^{k-1} Q_{j}(\Delta t A) y^{n-j}
$$

must be solved. A preconditioned fixed point iteration begins with Eq. (12) in the form

$$
y^{n+1}=Q_{-1}(\Delta t A) y^{n+1}+\sum_{j=0}^{k-1} Q_{j}(\Delta t A) y^{n-j} .
$$

Note that for this linear problem, the Jacobi and Gauss Seidel preconditioners (Eqs. (5) and (6)) are exactly the Jacobi and Gauss Seidel matrix splittings applied to the matrix 
$Q_{-1}(\Delta t A)$. More precisely, for the Jacobi case, the matrix is split $Q_{-1}(\Delta t A)=D-O$, where $D$ is the diagonal and $-O$ is the off-diagonal. The iteration is performed:

$$
\left(y^{n+1}\right)^{s}=D\left(y^{n+1}\right)^{s}-O\left(y^{n+1}\right)^{s-1}+\sum_{j=0}^{k-1} Q_{j}(\Delta t A) y^{n-j}, s=1,2, \ldots
$$

Rearranging to solve for $\left(y^{n+1}\right)^{s}$ gives

$$
(I-D)\left(y^{n+1}\right)^{s}=-O\left(y^{n+1}\right)^{s-1}+\sum_{j=0}^{k-1} Q_{j}(\Delta t A) y^{n-j},
$$

which is exactly the Jacobi splitting of the matrix of the linear system in Eq. (12). Thus, for a linear problem, preconditioned time differencing is a first order linear iterative method [47], where the implicit matrix is split $\left[I-Q_{-1}(\Delta t A)\right]=M-N$ and the solution $y^{n+1}$ is obtained by the iteration

$$
M\left(y^{n+1}\right)^{s}=N\left(y^{n+1}\right)^{s-1}+\sum_{j=0}^{k} Q_{j}(\Delta t A) y^{n-j}, s=1,2, \ldots
$$

The method is convergent (i.e. contractive) if and only if the spectral radius of $M^{-1} N$ is less than 1 [26].

As an example, consider the Backward Euler method

$$
y^{n+1}=\Delta t \quad A y^{n+1}+y^{n} .
$$

A fixed point iteration for solving the implicit system of equations (13) would fail to converge unless a restrictive $\Delta t$ is taken since the eigenvalues of $A$ are essentially $\lambda \cong-a,-\varepsilon$. The Jacobi preconditioner (Eq. (5)) applied to Eq. (13) using the identity predictor (Eq.(7)) when only one iteration is taken (cf. algorithm 1.1) has the form $M y^{n+1}=(N+I) y^{n}$, where $M=\left[\begin{array}{cc}1+a \Delta t & 0 \\ 0 & 1+\varepsilon \Delta t\end{array}\right], \quad(N+I)=\left[\begin{array}{cc}1 & -\varepsilon \Delta t \\ a \Delta t & 1\end{array}\right]$. 
This method will be stable if the spectral radius of $M^{-1}(N+I)$ is less than unity. Now, it is easy to see that the eigenvalues of this matrix are approximately

$\lambda=(1+a \Delta t)^{-1},(1+\varepsilon \Delta t)^{-1}$, which are clearly less than 1 . The exact eigenvalues are graphed as functions of the stepsize $\Delta t$ in Figure 1. Unconditional stability may therefore be expected using the Jacobi preconditioner (Eq. (5)) on the nonlinear problem (to the extent that this linear approximation applies). Other preconditioning schemes can be analyzed in this fashion.

\subsubsection{Numerical Experiments: The Nonlinear Chapman Mechanism}

The preceding section dealt only with the linear initial value problem approximating the Chapman mechanism. The full nonlinear Chapman mechanism is solved using the Jacobi and Gauss-Seidel preconditioners Eqs. (5) and (6). The underlying implicit difference methods here are the first order Backward Euler method (Eq. (3)) and the second order trapezoid rule (Eq. (4)). Experiments with one or two iterations are carried out. A variable time step is used, where the stepsize is adjusted by examining the difference in the calculated species values from a single step $\left(y^{n+1}(\Delta t)\right)$ with that taken from two half steps $\left(y^{n+1}\left(\frac{\Delta t}{2}\right)\right)$. This difference provides the highest order term in the error; even though this is an expensive method for stepsize control, it is the only accurate means for allowing the stepsize to vary. More precisely, note that (in the case of a first order accurate method, such as backward Euler) $y^{n+1}(\Delta t) \approx y\left(t_{n+1}\right)+C(\Delta t)^{2}$ for some constant $C$. The assumption is made, that for the same constant $y^{n+1}\left(\frac{\Delta t}{2}\right) \approx y\left(t_{n+1}\right)+2 C\left(\frac{\Delta t}{2}\right)^{2}$. Thus, when the two are subtracted, the quantity

$$
E^{n+1}=y^{n+1}(\Delta t)-y^{n+1}\left(\frac{\Delta t}{2}\right) \approx \frac{C(\Delta t)^{2}}{2}=2 C\left(\frac{\Delta t}{2}\right)^{2}
$$


FIGURE 1. Plots of the eigenvalues of the transition matrix $M^{-1}(N+I)$ from the Jacobi preconditioned backward Euler example on the linearized Chapman model (1a and 1b)

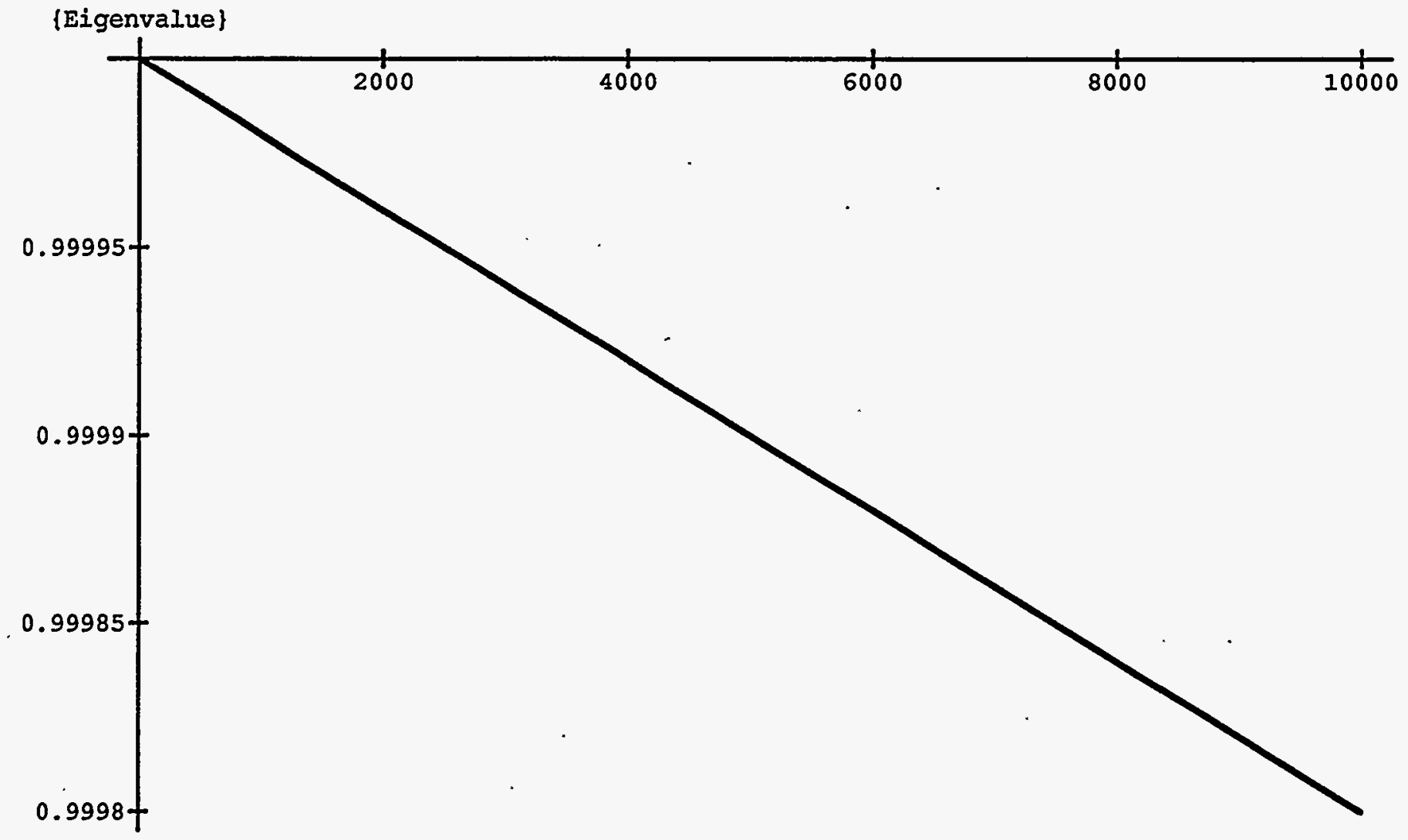


(Figure 1b--Continuation of Figure 1)

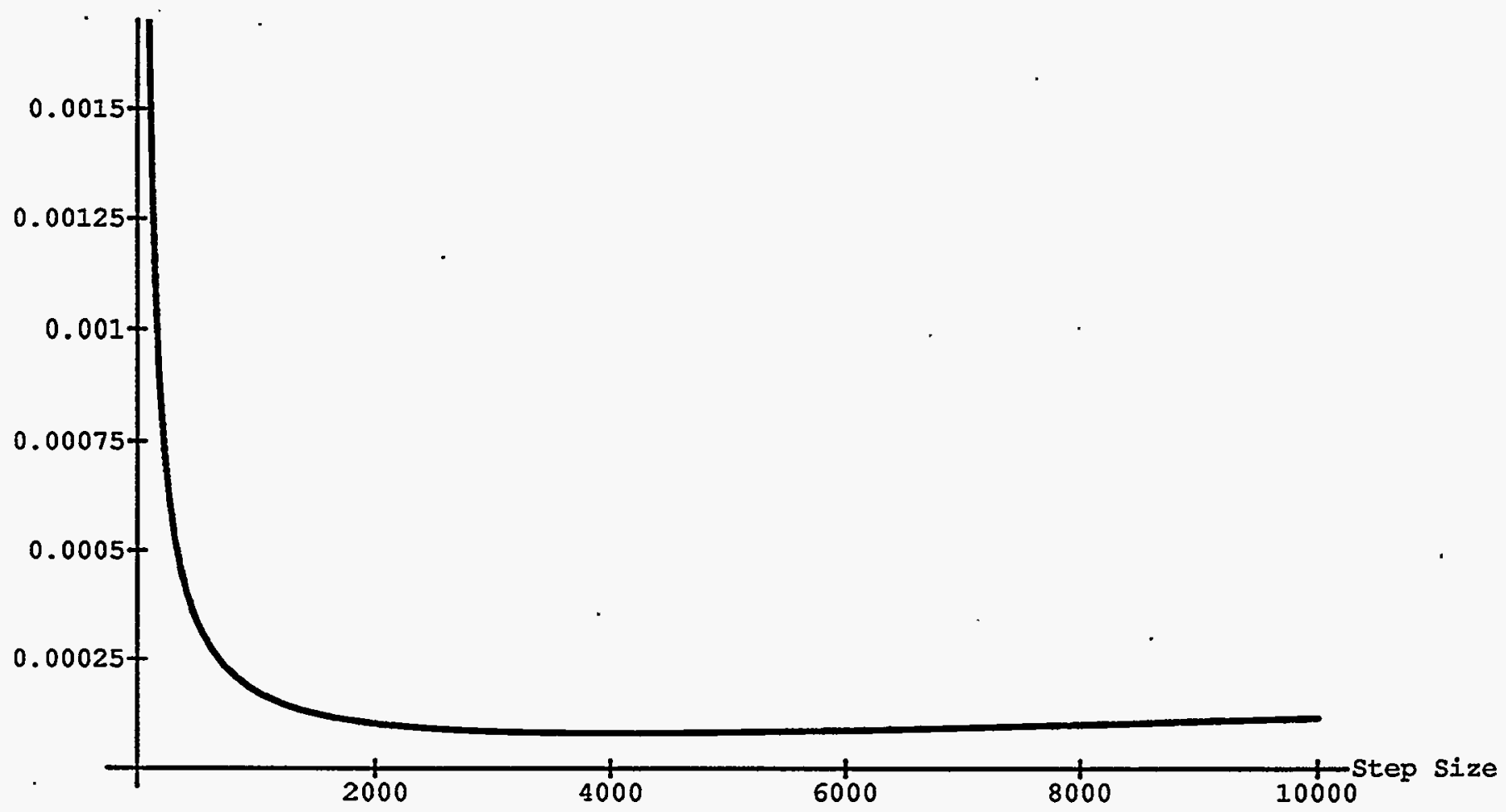


is an approximation to the truncation error in $y^{n+1}\left(\frac{\Delta t}{2}\right)$. A higher order method works the same way: a $p$ th order method has a truncation error that is $O\left((\Delta t)^{p+1}\right)$ instead of . $O\left((\Delta t)^{2}\right)$ as in this first order example [45, pp.708-716].

The stepsize is adjusted by defining

$$
\max _{i}\left(\frac{E_{i}^{n+1}}{\operatorname{ATOL}(I)+R T O L(I) y_{i}^{n}}\right) \equiv\left\|E^{n+1}\right\|,
$$

where ATOL and RTOL are arrays containing the absolute and relative error tolerances respectively. That is to say, the user desires the local truncation error to be less than $\operatorname{ATOL}(I)+R T O L(I) y_{i}^{n}$ for each $i$, at each step. Hence, ATOL specifies a portion of the error as an absolute parameter, while RTOL specifies the remaining portion as a percentage fraction of the species value. This method of specifying error tolerance is common in ODE solvers of this type $[3,9,29,46,67]$. Pure relative error control can be obtained by setting ATOL to zero. RTOL then gives a measure of the number of significant digits that is desired for the solution. It is a problem, however, if the solution vanishes, as the relative error then becomes undefined. It is similarly possible to obtain pure absolute error control by setting RTOL to zero. ATOL is then a measure of the largest number that can be neglected by the solver. This method of control is inappropriate if the solution is expected to change by many orders of magnitude. In chemical kinetics, the solutions commonly vary by orders of magnitude and sometimes vanish (or at least nearly so). This is why a "hybrid" error control is necessary. Thus, if the weighted norm $\left\|E^{n+1}\right\|$ is less than one, the step is accepted. The next stepsize to be attempted is calculated via the expression

$$
\Delta t_{\text {new }}=\left(\frac{0.94}{p+1 \sqrt[1]{\left\|E^{n+1}\right\|}}\right) \Delta t_{\text {old }}
$$


where $p$ is again the order of the integration scheme. The stepsize is not allowed to be increased more than a factor of two for any single adjustment. Whenever a step is rejected, the stepsize is subsequently not allowed to be increased by more than five percent in any single adjustment. When at least ten successive steps are accepted, the stepsize is again allowed to increase by as much as a factor of two. This scheme prevents the method from increasing the step length too aggressively.

\subsubsection{First Order Backward Euler, Jacobi Preconditioner}

Preconditioned time differencing for the nonlinear Chapman Mechanism is illustrated by considering the Jacobi preconditioner (Eq. (5)) and the identity predictor (Eq. (7)) as applied to the backward Euler method (Eq. (3)). This yields, in the case of a single iteration, the component equations (use algorithm 1.1, apply to Eq. (10) and recall that $\bar{L}=0$ )

$$
y_{i}^{n+1}=\left(y_{i}^{n+1}\right)^{1}=y_{i}^{n}+\Delta t\left(P_{i}^{n}-\hat{L}_{i}^{n} y_{i}^{n+1}\right)
$$

where $P_{i}^{n}, \hat{L}_{i}^{n}$ indicate that these values are calculated using old values of the $y_{j}, j \neq i$. It is then easy to solve for $y_{i}^{n+1}$ explicitly:

$$
y_{i}^{n+1}=\frac{y_{i}^{n}+\Delta t P_{i}^{n}}{1+\Delta t \hat{L}_{i}^{n}}
$$

It is not difficult to verify by Taylor expansion that Eq. (14) retains first order accuracy. Note also that Eq. (14) looks very much like the situation

$$
y^{n+1}=M^{-1}\left(y^{n}+b\right)
$$

with $M^{-1}$ a diagonal matrix having $\left(1+\Delta t \hat{L}^{n}\right)^{-1}$ on its main diagonal (assuming these values are constant). These "eigenvalues" are clearly less than one, which is suggestive that one may expect unconditional stability for this type of nonlinear problem. Multiple iterations of the preconditioner in algorithm 1.1 are implemented in the obvious way: 


$$
\left(y_{i}^{n+1}\right)^{s+1}=\frac{y_{i}^{n}+\Delta t\left(P_{i}^{n+1}\right)^{s}}{1+\Delta t\left(\hat{L}_{i}^{n+1}\right)^{s}}
$$

Figures 2 and 3 show graphs of the average step length in seconds during a given hour versus the time in hours. All runs began at sunrise and end at sunset (hours 0 and 12 respectively). These graphs show the large stepsizes that this method is capable of taking. The average step starts out quite small and builds throughout the day, as the chemistry approaches equilibrium. In the latter part of the day, the diurnal rates are gradually turned off. This causes species to change, and is seen in the dropoff of the stepsize in the last six ' hours of these graphs. Investigation into the use of more than one iteration of the preconditioner (Eq. (15)) showed that second iteration seemed to allow a larger average step (Fig. 3), but this effect quickly diminished. The average step taken by the Jacobi preconditioner after twenty iterations is no better than that permitted by a single iteration.

In Tables I and II CPU times and accuracy data are given for the ozone species (the second component of the dependent variable) solved with one and two iterations of this method. In terms of CPU time, these were the most successful versions of this first order method. Each column represents a run using the value of RTOL specified at the head of the column. The first row shows the accuracy in terms of the significant digits delivered, the second row shows the CPU time spent, and the third row shows the total number of numerical steps taken. The initial time is zero (sunrise) and the finish time is 43,200 (sunset). ATOL is set to 0.01 for all runs. The number of significant digits is calculated by taking the log of the relative (percent) error at the finish time.

TABLE I. Ozone solved using the backward Euler method with the Jacobi preconditioner, one iteration, and the identity predictor.

\begin{tabular}{|l|l|l|l|}
\hline RTOL & $10^{-2}$ & $10^{-4}$ & $10^{-6}$ \\
\hline Sig. Digits & 1.43 & 1.63 & 5.03 \\
\hline CPU time & 0.3 units & 0.9 units & 18.4 units \\
\hline Steps & 587 & 1505 & 33571 \\
\hline
\end{tabular}


FIGURE 2. Backward Euler method with the Jacobi preconditioner, one Iteration, and the identity predictor. Average step length versus hour.

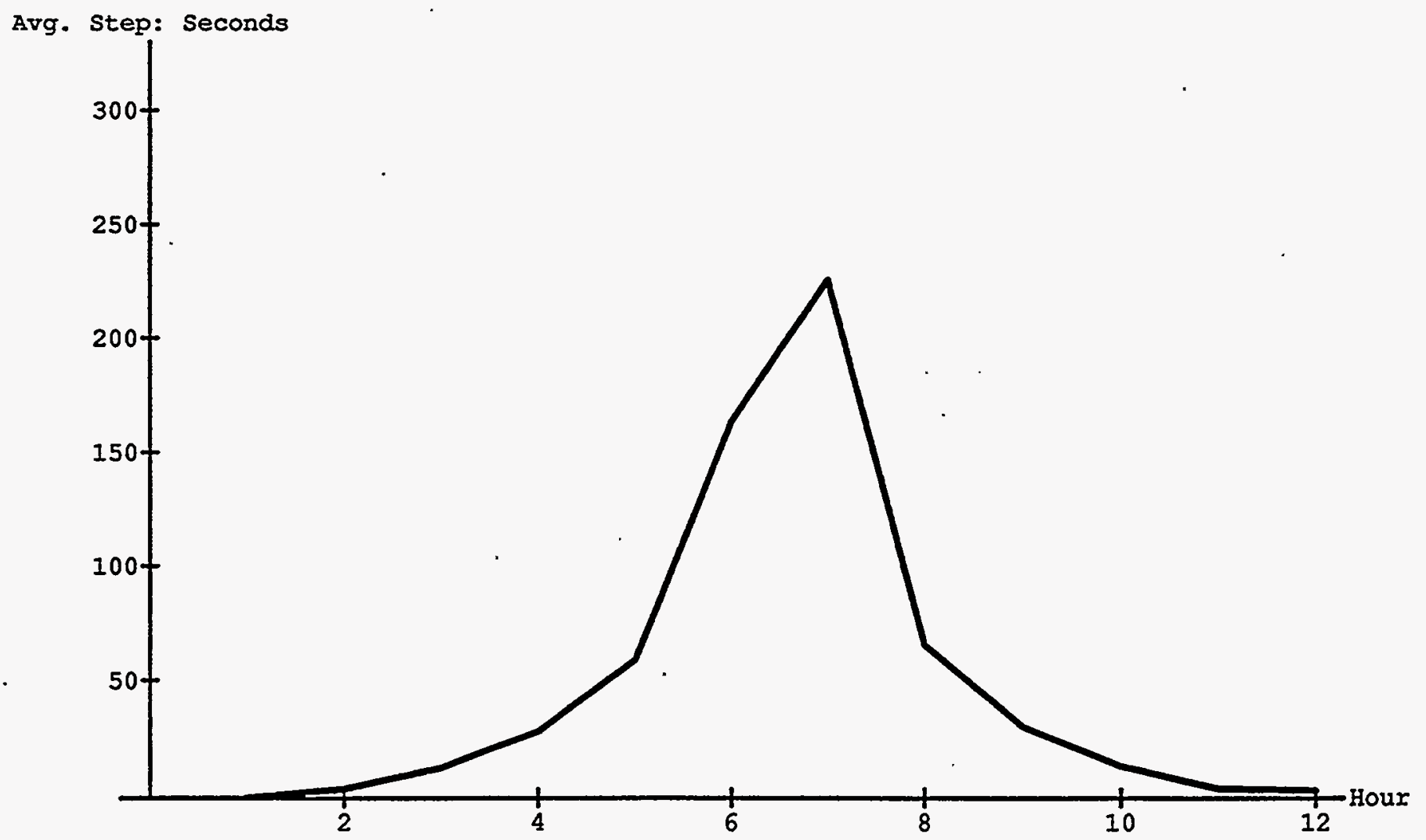


FIGURE 3. Backward Euler method with the Jacobi preconditioner, two Iterations, and the identity predictor. Average step length versus hour.

Avg. Step: Seconds

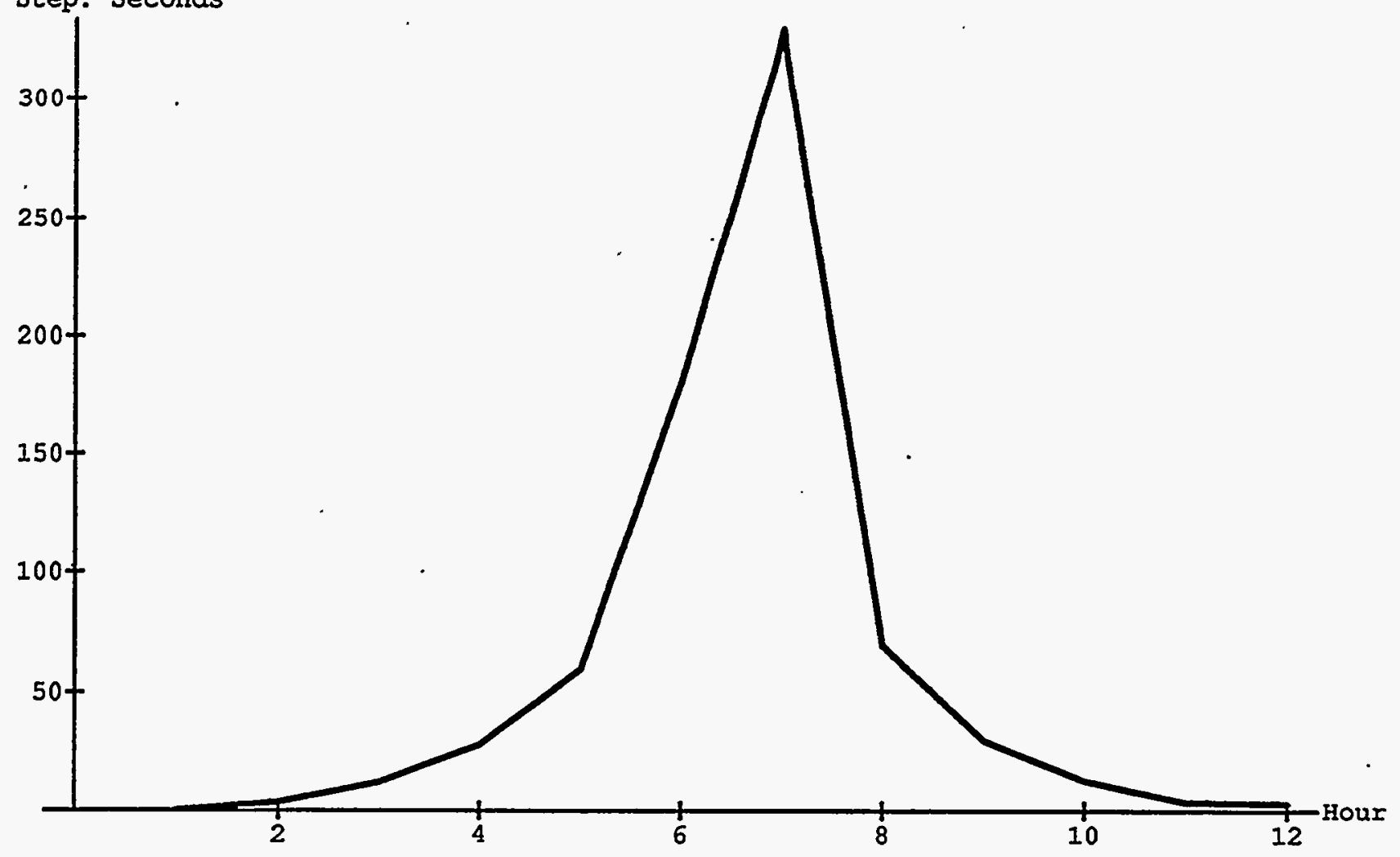


TABLE II. Ozone solved using the backward Euler method with the Jacobi preconditioner, two iterations, and the identity predictor.

\begin{tabular}{|l|l|l|l|}
\hline RTOL & $10^{-2}$ & $10^{-4}$ & $10^{-6}$ \\
\hline Sig. Digits & 2.02 & 2.94 & 3.92 \\
\hline CPU time & 0.6 units & 1.4 units & 8.2 units \\
\hline Steps & 608 & 1508 & 8618 \\
\hline
\end{tabular}

Interesting to note in Table $I$ is that the method struggles when asked to provide higher. accuracy answers (column three, this could be a result of the cautious manner in which the stepsize was increased). This is not much of a handicap in atmospheric kinetics, as greater than $1 \%$ accuracy in solving the kinetics equations is generally considered redundant $[65$, 66]. Table II presents results obtained using the same parameters as in Table I but with two iterations of the preconditioner (cf. Eq. (15)). The number of steps remains about the same (except for the high accuracy data in column three) but accuracy and run times are increased.

Figure 4 shows a full 48 hour run of the Chapman mechanism. At sunrise and sunset (hours $0,24,48$ and 12,36 ) photochemical reactions are turned on and off respectively. At these times, reaction rates and species concentrations change sharply, forcing very small timesteps in order to resolve the behavior. The stepsize does build throughout the day, characteristic of an unconditionally stable method. During the nighttime hours, the method manages timesteps of one hour (the set maximum allowed). This indicates that the step control is behaving properly.

\subsubsection{First Order Backward Euler, Gauss-Seidel Preconditioner}

The Gauss-Seidel scheme is devised by recalculating the production and loss terms with successively more accurate values as species are calculated (cf. Eq. (6)). Again, numerical experiments show the scheme to be unconditionally stable. 
A study of figures 5 and 6 shows that a Gauss-Seidel preconditioner can outperform the Jacobi method, in terms of the average step taken. This performance, though, is understandably affected by the order in which the updates are done, since the species that are updated first are computed with less accurate information than those that are updated last. The Gauss-Seidel method clearly outperforms Jacobi when atomic oxygen is updated last (Fig. 6), but seems to have little if any advantage when oxygen is updated first (Fig. 5). This indicates that the Gauss-Seidel method is preferable if the equations are arranged in the most efficient order. Since there seems to be no clear way of ordering the equations apriori, the Gauss-Seidel could be detrimental. Not easily seen in these figures is the larger number of steps that the Gauss Seidel method takes at start-up (sunrise). This is when the diurnally varying rates are activated and seems to demonstrate the difficulty Gauss Seidel has with the diurnal effect (this is clearly seen in the total number of steps taken in the following tables). Actual CPU times for the Gauss-Seidel schemes show a clear advantage for the Jacobi method (Tables III, IV, V, and VI).

TABLE III. Ozone solved using the backward Euler method and Gauss-Seidel preconditioner, one iteration. Atomic oxygen updated first.

\begin{tabular}{|l|l|l|l|}
\hline RTOL & $10^{-2}$ & $10^{-4}$ & $10^{-6}$ \\
\hline Sig. Digits & 1.51 & 3.56 & 5.43 \\
\hline CPU time & 1.4 units & 2.6 units & 39.6 units \\
\hline Steps & 1242 & 2138 & 33799 \\
\hline
\end{tabular}

TABLE IV. Ozone solver using the backward Euler method and Gauss-Seidel preconditioner, one iteration. Atomic oxygen updated last.

\begin{tabular}{|l|l|l|l|}
\hline RTOL & $10^{-2}$ & $10^{-4}$ & $10^{-6}$ \\
\hline Sig. Digits & 3.26 & 4.08 & 4.08 \\
\hline CPU time & 1.5 units & 2.5 units & 10.1 units \\
\hline Steps & 1267 & 2092 & 8490 \\
\hline
\end{tabular}


FIGURE 4. Jacobi preconditioned backward Euler method, one iteration, using the identity predictor. (48 hour run). Average step length versus hour.

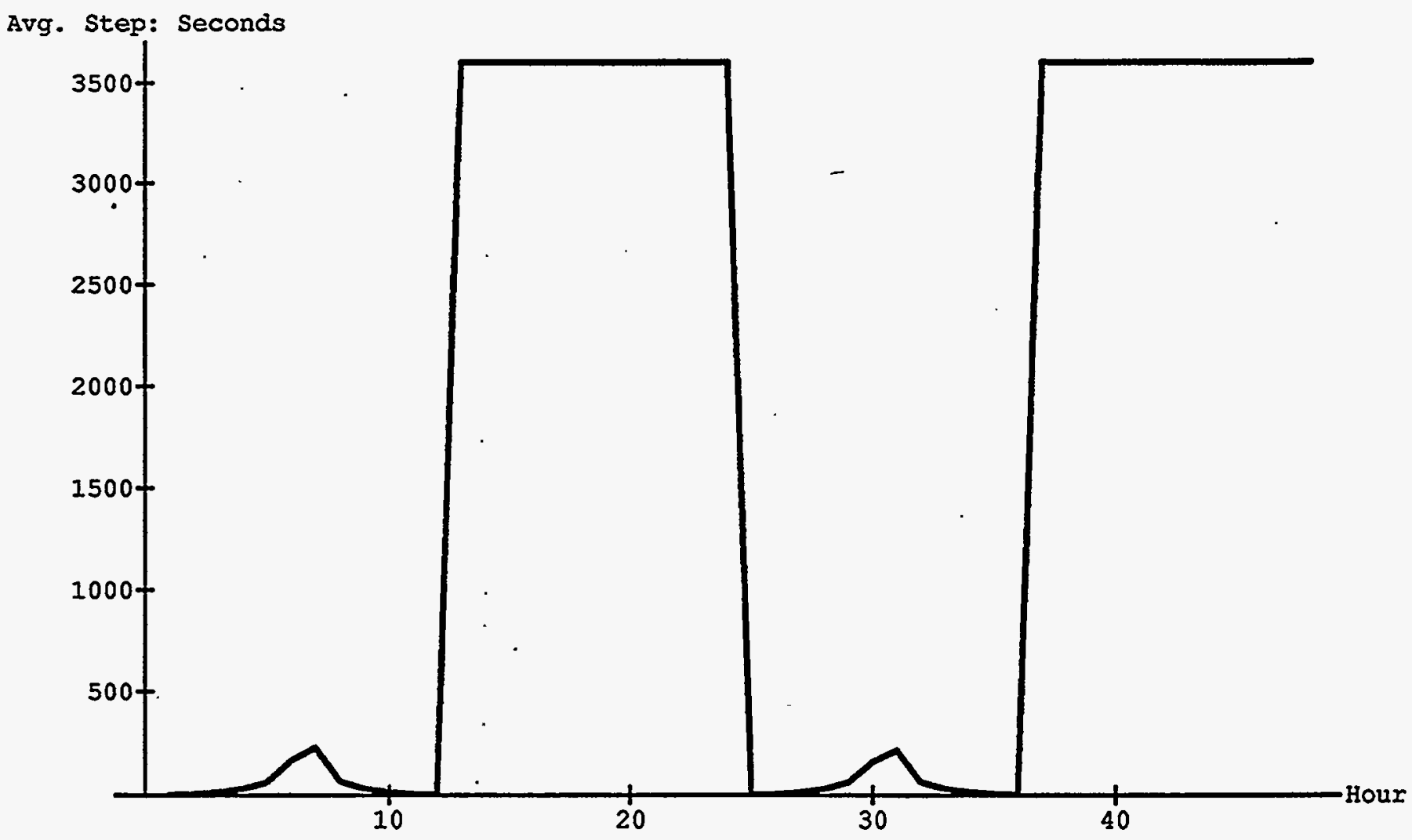


FIGURE 5. Backward Euler method with the Gauss-Seidel preconditioner, one iteration, identity predictor. Atomic oxygen updated first. Average step length versus hour.

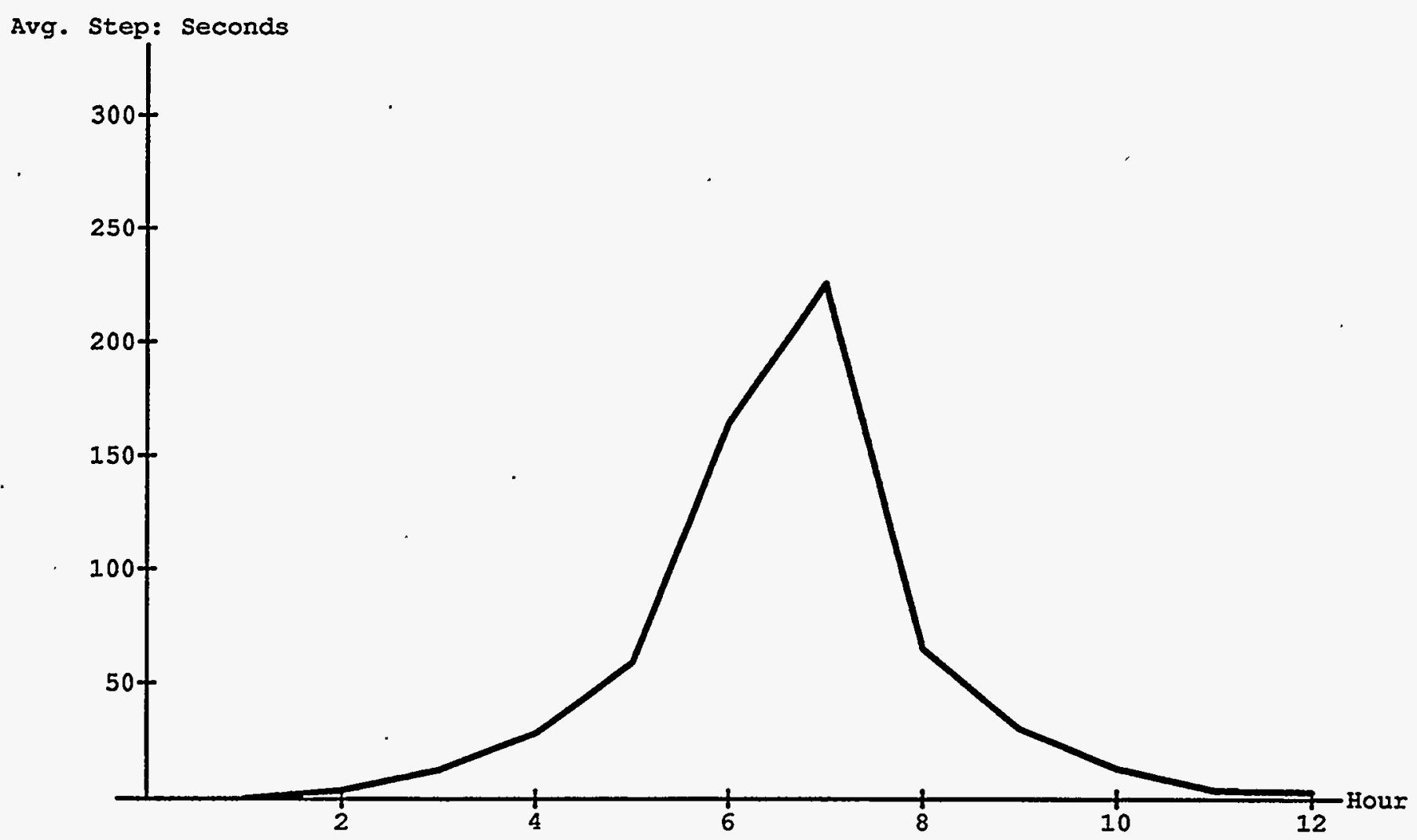


FIGURE 6. Backward Euler method with the Gauss-Seidel preconditioner, one iteration, identity predictor. Atomic oxygen updated last. Average step length versus hour.

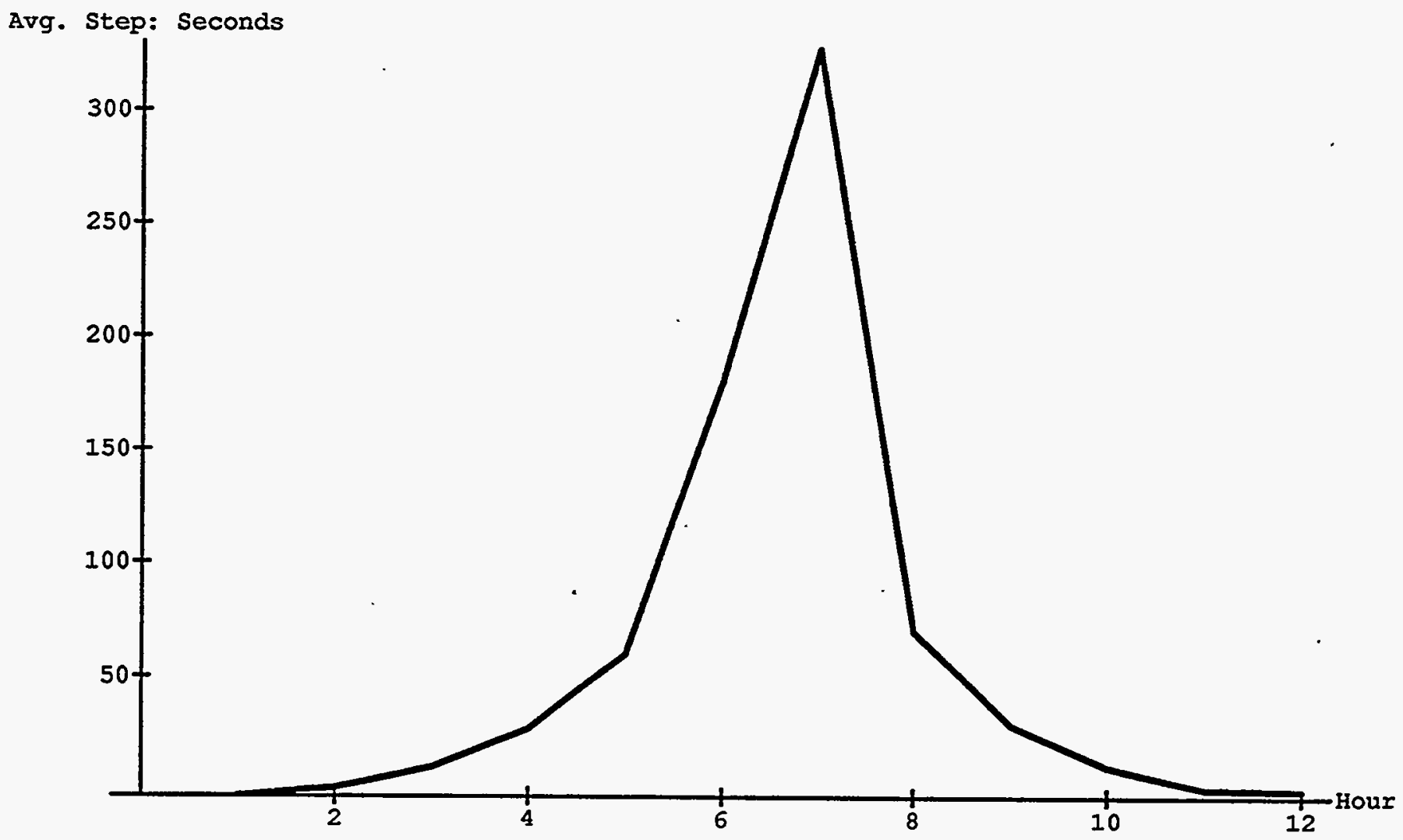


TABLE V. Ozone solved using the backward Euler method and Gauss-Seidel preconditioner, two iterations. Atomic oxygen updated first.

\begin{tabular}{|l|l|l|l|}
\hline RTOL & $10^{-2}$ & $10^{-4}$ & $10^{-6}$ \\
\hline Sig. Digits & 1.93 & 3.33 & 4.25 \\
\hline CPU time & 2.7 units & 3.9 units & 12.8 units \\
\hline Steps & 1236 & 1782 & 5684 \\
\hline
\end{tabular}

TABLE VI. Ozone solver using the backward Euler method and Gauss-Seidel preconditioner, two iterations. Atomic oxygen updated last.

\begin{tabular}{|l|l|l|l|}
\hline RTOL & $10^{-2}$ & $10^{-4}$ & $10^{-6}$ \\
\hline Sig. Digits & 2.37 & 4.13 & 5.03 \\
\hline CPU time & 2.7 units & 4.0 units & 12.4 units \\
\hline Steps & 1257 & 1822 & 5518 \\
\hline
\end{tabular}

In summary, the Jacobi and Gauss-Seidel preconditioners (Eqs. (5) and (6)) applied to the Backward Euler method (Eq. (3)) with the "identity predictor" (Eq. (7)) yield first order accurate, unconditionally stable, explicit methods for this problem (cf. algorithm 1.1). The large stepsizes together with the small computational overhead (explicit calculations) show considerable promise for speedup over the implicit Newton solvers currently in use on larger problems. The next focus is the development of a second order method.

\subsubsection{Second Order Trapezoid Rule}

The implementation of a second order method begins with the trapezoid rule (Eq. (4)). The Jacobi preconditioner (Eq. (5)) is applied, taking only one or two iterations, as this combination was most successful (in terms of CPU time and robustness) in the last section. Even though the trapezoid rule is not the best choice for a very stiff problem, a onestep method is desired at this early stage, as the one-step property would be very memory efficient on larger problems. This yields (in the case of only a single iteration)

$$
y_{i}^{n+1}=\frac{\Delta t}{2}\left[\left(P_{i}^{n}-\hat{L}_{i}^{n} y_{i}^{n+1}-\bar{L}_{i}^{n}\left(y_{i}^{n+1}\right)^{2}\right)+\left(P_{i}^{n}-\hat{L}_{i}^{n} y_{i}^{n}-\bar{L}_{i}^{n}\left(y_{i}^{n}\right)^{2}\right)\right]+y^{n}
$$


where the identity predictor (Eq. (7)) has been used as in the last section. A Taylor expansion reveals that the method (16) is only first order accurate. At least a first order predictor must be used as the initial guess (step 1 in algorithm 1.1) in order to preserve the second order accuracy of the trapezoid rule [4]. The explicit, first order accurate Jacobi preconditioned Backward Euler method (Eq. (14)) of section 1.1.3 is used.

The second order preconditioned time differencing method is then:

\section{Algorithm 1.2}

1. Set $\left(y_{i}^{n+1}\right)^{(0)}=\frac{y_{i}^{n}+\Delta t P_{i}^{n}}{1+\Delta t \hat{L}_{i}^{n}}$.

2. Calculate $P^{n+1}$ and $\hat{L}^{n+1}$ using these values.

3. $y_{i}^{n+1}=\left(y_{i}^{n+1}\right)^{1} \cdot=\frac{\frac{\Delta t}{2}\left[\left(P_{i}^{n+1}\right)+\left(P_{i}^{n}-\hat{L}_{i}^{n} y_{i}^{n}\right)\right]+y^{n}}{1+\frac{\Delta t}{2} \hat{L}_{i}^{n+1}}$

Multiple iterations of the preconditioner are again implemented in the obvious way as in Eq. (15).

This method, like the first order preconditioned scheme, seems to have good stability characteristics. A table of results for ozone (Table VII), similar to Table I is presented here. The parameters for the problem are the same, as are all error control parameters.

A Gauss Seidel scheme is possible in the same manner as with the Backward Euler method (in this case the Gauss Seidel Preconditioned Backward Euler method is used as the predictor in step 1). A comparison of the Jacobi and Gauss Seidel preconditioners leads to results similar to those in the previous section. Gauss Seidel run times are significantly longer using the same error control parameters. 
The results for multiple iterations of the preconditioner reduced the number of steps taken, but nearly doubled the CPU time for this problem. As the methods go to higher order, the iterations are more expensive and provide diminishing returns [4].

Tables VIII and IX show. Gauss Seidel runs and, as in the last section, show a clear advantage for the Jacobi method.

TABLE VII. Ozone solved using the trapezoid rule with the Jacobi preconditioner, one iteration, and the Jacobi preconditioned backward Euler method as a predictor.

\begin{tabular}{|l|l|l|l|}
\hline RTOL & $10^{-2}:$ & $10^{-4}$ & $10^{-6}$ \\
\hline Sig. Digits & 2.17 & 3.02 & 3.99 \\
\hline CPU time & 0.6 units & 0.9 units & 4.7 units \\
\hline Steps & 315 & 488 & 2964 \\
\hline
\end{tabular}

TABLE VIII. Ozone solved using the trapezoid rule with the Gauss-Seidel preconditioner, one iteration, and the Gauss-Seidel preconditioned backward Euler method as a predictor. Atomic oxygen updated first.

\begin{tabular}{|l|l|l|l|}
\hline RTOL & $10^{-2}$ & $10^{-4}$ & $10^{-6}$ \\
\hline Sig. Digits & 1.71 & 2.36 & 3.33 \\
\hline CPU time & 2.7 units $\cdot$ & 3.0 units & 3.8 units \\
\hline Steps & 1132 & 1167 & 1503 \\
\hline
\end{tabular}

TABLE IX. Ozone solved using the trapezoid rule with the Gauss-Seidel preconditioner, one iteration, and the Gauss-Seidel preconditioned backward Euler method as a predictor. Atomic oxygen updated last.

\begin{tabular}{|l|l|l|l|}
\hline RTOL & $10^{-2}$ & $10^{-4}$ & $10^{-6}$ \\
\hline Sig. Digits & 1.84 & 3.99 & 6.03 \\
\hline CPU time & 2.7 units & 3.1 units & 3.8 units \\
\hline Steps & 1122 & 1246 & 1530 \\
\hline
\end{tabular}

As a final accuracy performance check, a comparison of the Jacobi preconditioned trapezoid.rule (1 iteration, detailed in Algorithm 1.2) with the second order Backward Differentiation Formula (BDF) [12] is presented. An integration of the Chapman mechanism from sunrise to sunset was performed using a constant stepsize of 50 seconds for both tech- 
niques ( 50 seconds was chosen because it was a reasonable average step length, cf. Table VII). Figure 7a is a plot of the concentration of atomic oxygen $\left(y_{1}\right)$ over time as computed by the preconditioned technique. In Figure $7 \mathrm{~b}$, the absolute relative difference between the value computed by the second order $\mathrm{BDF}$ and the preconditioned technique is shown. The difference between the two is generally much less than five percent, the exception being when the species concentration goes to zero (the relative difference is undefined when the species value is zero). This demonstrates that the preconditioned schemes possess accuracy comparable to the benchmark schemes for stiff equations. Given an accurate method of step control, they should take nearly the same number of steps for the same problems. Since the preconditioned schemes are much less expensive to compute, they should clearly be the superior techniques in terms of CPU time.

When diurnal effects are not considered, Gauss Seidel iteration has been shown to be very efficient [65]; however, as the preceding computations demonstrate, the diurnal effects hurt the Gauss Seidel technique's performance. The Jacobi iteration is the clear choice when CPU time is the metric. In the next section, hydrogen and water vapor chemistry is added to the equations. This provides a more difficult problem to examine the preconditioned techniques, and confirm these results before proceeding with the final evaluation. 
FIGURE 7. (7a): Plot of atomic oxygen vs. time from the Chapman problem. Computed using the Jacobi preconditioned trapezoid rule (1 iteration) and a constant stepsize of 50 seconds. (7b): Plot of the relative difference between the computed values in (7a) and the computed values from the second order BDF using a constant stepsize of 50 seconds.

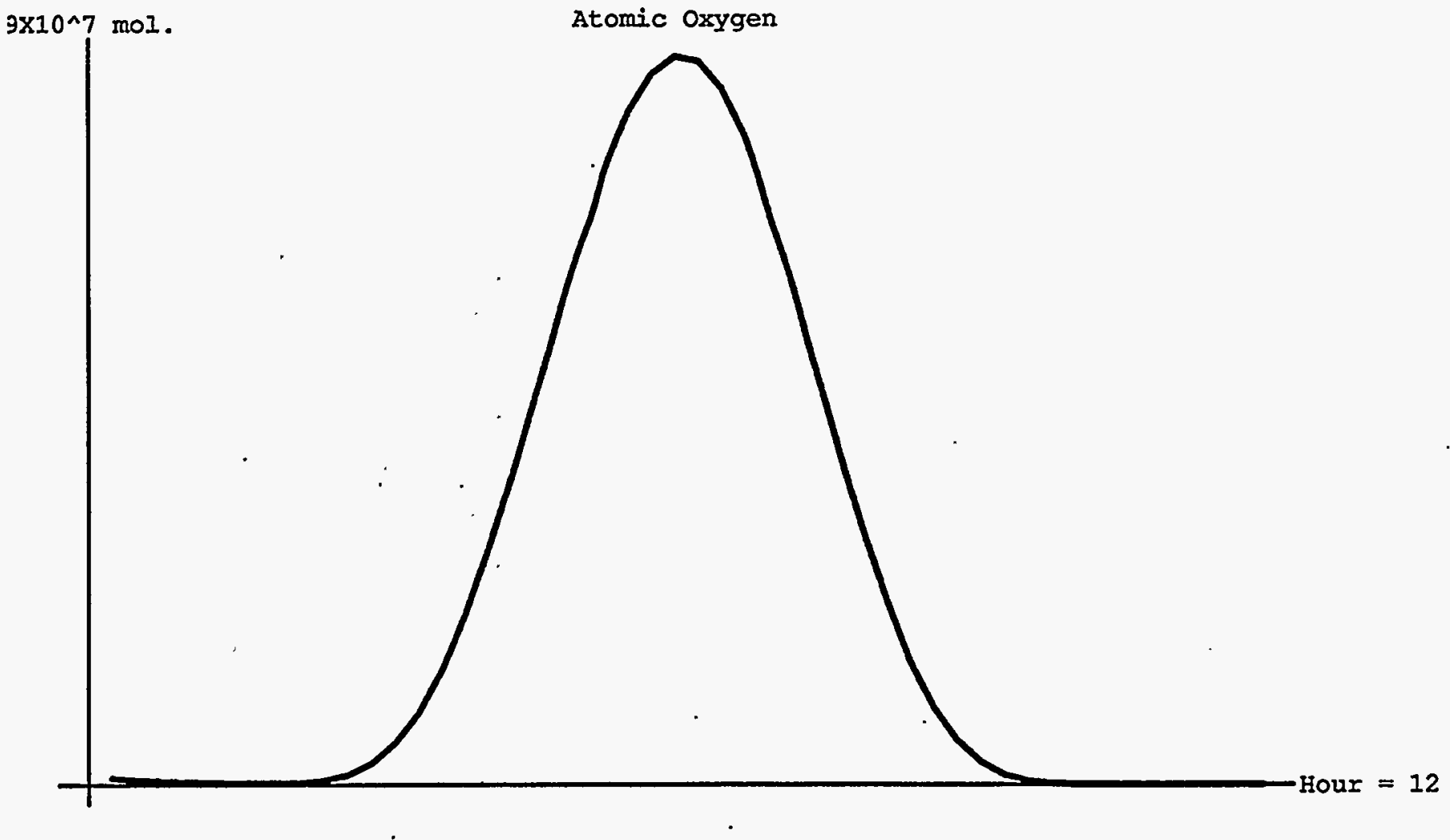


48

(Figure 7b--Continuation of Figure 7)

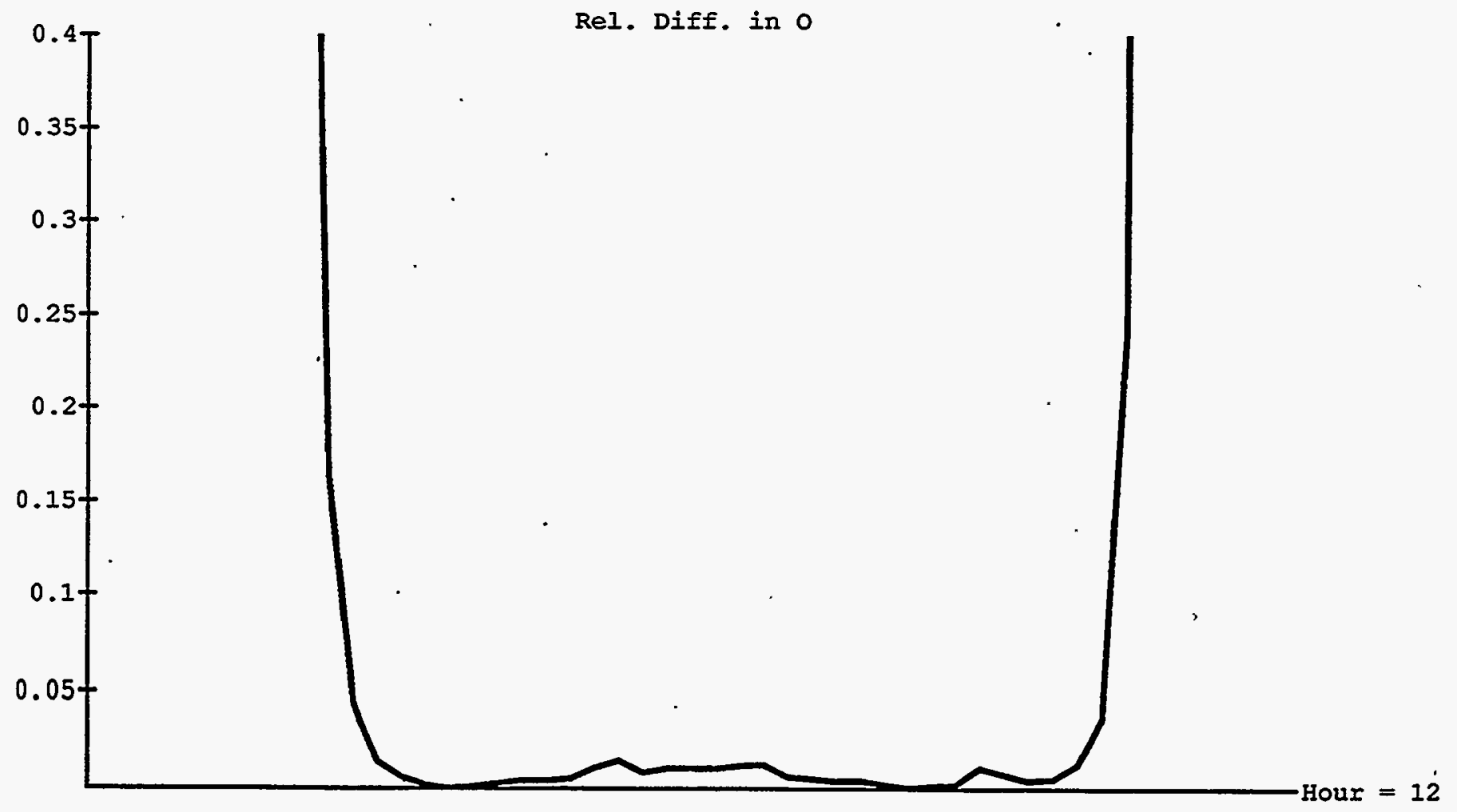




\subsection{More Complexity: Hydrogen Chemistry}

The preceding Chapman problem was quasi-linear and easily produced an explicit calculation when treated with the various preconditioned time differencing techniques. In this section a slightly more complicated mechanism that includes the quadratic behavior found in many reaction problems is used (i.e. $\bar{L} \neq 0$ ).

The following chemical species are included in the mechanism: (which are represented by the variables $\left.y_{i}, i=1, \ldots, 9\right) \mathrm{O}, \mathrm{O}_{3}, \mathrm{OH}, \mathrm{HO}_{2}, \mathrm{H}_{2} \mathrm{O}_{2}, \mathrm{~N}_{2}, \mathrm{O}_{2}, \mathrm{H}_{2} \mathrm{O}, \mathrm{O}\left({ }^{1} \mathrm{D}\right)$. The mechanism itself consists of 15 chemical reactions:

\begin{tabular}{lll|l}
\hline \hline $\mathrm{O}+\mathrm{O}_{2}+M$ & $\rightarrow$ & $\mathrm{O}_{3}+\mathrm{M}$ & $k_{1}$ \\
$\mathrm{O}+\mathrm{O}_{3}$ & $\rightarrow$ & $2 O_{2}$ & $k_{2}$ \\
$\mathrm{O}\left({ }^{1} \mathrm{D}\right)+\mathrm{N}_{2}$ & $\rightarrow$ & $\mathrm{O}+\mathrm{N}_{2}$ & $k_{3}$ \\
$\mathrm{O}\left({ }^{1} \mathrm{D}\right)+\mathrm{O}_{2}$ & $\rightarrow$ & $\mathrm{O}+\mathrm{O}_{2}$ & $k_{4}$ \\
$\mathrm{O}\left({ }^{1} \mathrm{D}\right)+\mathrm{H}_{2} \mathrm{O}$ & $\rightarrow$ & $2 \mathrm{OH}$ & $k_{5}$ \\
$\mathrm{OH}+\mathrm{O}_{3}$ & $\rightarrow$ & $\mathrm{HO}_{2}+\mathrm{O}_{2}$ & $k_{6}$ \\
$\mathrm{HO}_{2}+\mathrm{O}$ & $\rightarrow$ & $\mathrm{OH}+\mathrm{O}_{2}$ & $k_{7}$ \\
$\mathrm{HO}_{2}+\mathrm{O}_{3}$ & $\rightarrow$ & $\mathrm{OH}+2 \mathrm{O}_{2}$ & $k_{8}$ \\
$\mathrm{HO}_{2}+\mathrm{OH}$ & $\rightarrow$ & $\mathrm{H}_{2} \mathrm{O}+\mathrm{O}_{2}$ & $k_{9}$ \\
$\mathrm{HO}_{2}+\mathrm{HO}_{2}$ & $\rightarrow$ & $\mathrm{H}_{2} \mathrm{O}_{2}+\mathrm{O}_{2}$ & $k_{10}$ \\
$\mathrm{H}_{2} \mathrm{O}_{2}+\mathrm{OH}$ & $\rightarrow$ & $\mathrm{H}_{2} \mathrm{O}+\mathrm{HO}_{2}$ & $k_{11}$ \\
$\mathrm{O}_{2}+h v$ & $\rightarrow$ & $2 O$ & $k_{12}$ \\
$\mathrm{O}_{3}+h v$ & $\rightarrow$ & $\mathrm{O}+\mathrm{O}_{2}$ & $k_{13}$ \\
$\mathrm{O}_{3}+h v$ & $\rightarrow$ & $\left.\mathrm{O}{ }^{1} \mathrm{D} \mathrm{D}\right)+\mathrm{O}_{2}$ & $k_{14}$ \\
$\mathrm{H}_{2} \mathrm{O}_{2}+h v$ & $\rightarrow$ & $2 \mathrm{OH}$ & $k_{15}$ \\
\hline \hline
\end{tabular}

The constant reaction rates are:

\begin{tabular}{|l|l|l|l|}
\hline$k_{1}=1.46 \times 10^{-16}$ & $k_{4}=4.20 \times 10^{-11}$ & $k_{7}=6.00 \times 10^{-11}$ & $k_{10}=2.30 \times 10^{-12}$ \\
$k_{2}=2.12 \times 10^{-15}$ & $k_{5}=2.20 \times 10^{-10}$ & $k_{8}=1.50 \times 10^{-15}$ & $k_{11}=1.50 \times 10^{-12}$ \\
$k_{3}=2.80 \times 10^{-11}$ & $k_{6}=3.70 \times 10^{-14}$ & $k_{9}=1.20 \times 10^{-10}$ & \\
\hline
\end{tabular}


The diurnally varying reaction rates are again mathematically idealized, and take the same form as in the Chapman problem (Eq. (11)) with:

\begin{tabular}{|l|l|l|l|}
\hline$a_{12}=22.62$ & $a_{13}=7.601$ & $a_{14}=7.500$ & $a_{15}=10.40$ \\
\hline
\end{tabular}

Again, $\omega=\frac{\pi}{43200}$.

Three of the species are assumed constant:

$y_{6}=1.3 \times 10^{17}, y_{7}=3.4 \times 10^{16}, y_{8}=9.4 \times 10^{11}$ and one is assumed to be in instantaneous equilibrium:

$$
y_{9}=\frac{k_{14} y_{2}}{k_{3} y_{6}+k_{4} y_{7}+k_{5} y_{8}}
$$

The five remaining species react according to a differential system which again has the general form of (Eq. (10)):

$$
\begin{array}{ll}
\frac{d y_{1}}{d t}= & \left(k_{3} y_{9} y_{6}+k_{4} y_{9} y_{7}+2 k_{12} y_{7}+k_{13} y_{2}\right)-\left(k_{1} y_{7}+k_{2} y_{2}+k_{7} y_{4}\right) y_{1} \\
\frac{d y_{2}}{d t}= & \left(k_{1} y_{1} y_{7}\right)-\left(k_{2} y_{1}+k_{6} y_{3}+k_{8} y_{4}+k_{13}+k_{14}\right) y_{2} \\
\frac{d y_{3}}{d t}= & \left(2 k_{5} y_{8} y_{9}+k_{7} y_{1} y_{4}+k_{8} y_{2} y_{4}+2 k_{15} y_{5}\right)-\left(k_{6} y_{2}+k_{9} y_{4}+k_{11} y_{5}\right) y_{3} \\
\frac{d y_{4}}{d t}= & \left(k_{6} y_{2} y_{3}+k_{11} y_{3} y_{5}\right)-\left(k_{7} y_{1}+k_{8} y_{2}+k_{9} y_{3}\right) y_{4}-\left(2 k_{10}\right) y_{4}^{2} \\
\frac{d y_{5}}{d t}= & \left(k_{10} y_{4}^{2}\right)-\left(k_{11} y_{3}+k_{15}\right) y_{5}
\end{array}
$$

with appropriate initial conditions being

$$
\begin{array}{|l|l|l|l|l|}
\hline y_{1}(0)=10^{6} & y_{2}(0)=10^{12} & y_{3}(0)=10^{6} & y_{4}(0)=2 \times 10^{6} & y_{5}(0)=10^{7} \\
\hline
\end{array}
$$




\subsubsection{Numerical Implementation}

Again, for illustration, the backward Euler method (Eq. (3)), Jacobi preconditioner (Eq. (5)), a single iteration, and the identity predictor (Eq. (7)) are used.

The method then takes the form (algorithm 1.1):

$$
y_{i}^{n+1}=y_{i}^{n}+\Delta t\left(P_{i}^{n}-\hat{L}_{i}^{n} y_{i}^{n+1}-\bar{L}_{i}^{n}\left(y_{i}^{n+1}\right)^{2}\right)
$$

The quadratic behavior in this problem necessitates the solution of a quadratic equation (17) to compute $y_{i}^{n+1}$. A quick analysis reveals that the roots of the quadratic equation are a positive and a negative real number. The obvious choice is to set $y_{i}^{n+1}$ to be the positive root [4].

At the expense of a square root function call (used in the quadratic formula to solve Eq. (17)), the preconditioned time differencing method again yields an explicit method. Additionally, a Taylor expansion shows the method (17) to be first order accurate. Finally, as the stepsize goes to infinity, the calculated value will remain bounded.

The implementation of the second order method proceeds along similar lines: set the predicted value using the first order method (step 1 in algorithm 1.2), calculate the predicted production and loss rates (step 2), and solve for $y_{i}^{n+1}$ using the quadratic formula (step 3). Other than the use of the quadratic formula to solve for the value of $y_{i}^{n+1}$, the numerical implementation is identical to that used in the last section (as in algorithm 1.2). For clarity, one iteration of the Jacobi preconditioner (Eq. (5)) applied to the trapezoid rule (Eq. (4)) is detailed here. This algorithm takes the form:

\section{Algorithm 1.3}

1. Solve $\hat{y}_{i}^{n+1}=y_{i}^{n}+\Delta t\left(P_{i}^{n}-\hat{L}_{i}^{n} \hat{y}_{i}^{n+1}-\bar{L}_{i}^{n}\left(\hat{y}_{i}^{n+1}\right)^{2}\right)$ for $\hat{y}_{i}^{n+1}$.

2. Calculate $P^{n+1}, \hat{L}^{n+1}, \bar{L}^{n+1}$ using the $\hat{y}_{i}^{n+1}$. 
3. Solve

$y_{i}^{n+1}=\frac{\Delta t}{2}\left[\left(P_{i}^{n+1}-\hat{L}_{i}^{n+1} y_{i}^{n+1}-\bar{L}_{i}^{n+1}\left(y_{i}^{n+1}\right)^{2}\right)+\left(P_{i}^{n}-\hat{L}_{i}^{n} y_{i}^{n}-\bar{L}_{i}^{n}\left(y_{i}^{n}\right)^{2}\right)\right]+y^{n}$

for $y_{i}^{n+1}$.

Runs using the same error control parameters as with the Chapman mechanism are presented here. Again, experiments with larger stepsizes confirm that preconditioning the backward Euler method with an "identity predictor" yields first order accurate, unconditionally stable, explicit methods for this problem. The same holds true for the second order trapezoid rule with the first order method as the initial guess.

\subsubsection{Timings for the 5 Species Problem}

The timings for the 5 species problem are summarized here. In these results, the number of significant digits was computed by taking the log of the relative error of the most inaccurate species at the finish time. In each case, this was either $\mathrm{OH}$ or $\mathrm{HO}_{2}$. These species are relatively rapidly evolving transients; ozone accuracies were significantly higher.

The backward Euler techniques were not extremely successful. CPU times rose considerably in comparison to the Chapman problem. Results for the preconditioned trapezoid rule were much better, with the Jacobi preconditioner again clearly outperforming the Gauss Seidel. The Jacobi trapezoid rule was the clear winner. CPU time and accuracy data are given in tables $\mathrm{X}$ and $\mathrm{XI}$.

TABLE X. Five species problem solved using the trapezoid rule with the Jacobi preconditioner, one iteration, and the Jacobi preconditioned backward Euler method as a predictor.

\begin{tabular}{|l|l|l|l|}
\hline RTOL & $10^{-2}$ & $10^{-4}$ & $10^{-6}$ \\
\hline Sig. Digits & 1.10 & 1.99 & 3.89 \\
\hline CPU time & 1.7 units & 8.1 units & 63.6 units \\
\hline Steps & 411 & 2161 & 17212 \\
\hline
\end{tabular}


TABLE XI. Five species problem solved using the trapezoid rule with the Jacobi preconditioner, two iterations; and the Jacobi preconditioned backward Euler method as a predictor.

\begin{tabular}{|l|l|l|l|}
\hline RTOL & $10^{-2}$ & $10^{-4}$ & $10^{-6}$ \\
\hline Sig. Digits & 2.27 & 2.29 & 3.85 \\
\hline CPU time & 3.6 units & 5.0 units & 12.5 units \\
\hline Steps & 421 & 688 & 1806 \\
\hline
\end{tabular}

Finally, in the same spirit as the comparison done at the end of section 1.1.5, the accuracy of the Jacobi preconditioned trapezoid rule (1 iteration, detailed in Algorithm 1.3) is again demonstrated with a constant step length of 50 seconds. Figure 8 a shows a plot of the concentration for $\mathrm{HO}_{2}$ (one of the two species the technique had trouble with) over time computed with the preconditioned method, while Figure $8 \mathrm{~b}$ shows the relative difference between the preconditioned scheme and the second order BDF analogous to Figure 7. Again, the preconditioned technique is in good agreement with the BDF, differing by around eight percent near sunrise, and much less than four percent thereafter. Again, the preconditioned techniques are of comparable accuracy to the counterpart BDFs commonly used to treat stiff problems.

This study has determined the successful variant of this technique to be used on chemical kinetics equations with strong diumal cycles. The next task is to establish this technique as an acceptable alternative to the most commonly used methods. In the next section, the Jacobi preconditioned trapezoid rule is compared with one of the more efficient, robust ODE solvers on a set of kinetics equations taken from a full chemical-radiative-transport model. 
FIGURE 8. (8a): Plot of HO2 vs. time from the 5 species problem using the same method as Figure 7. (8b): Corresponding plot of the relative difference between the values in (8a) and those obtained from the second order BDF using a constant stepsize of $\mathbf{5 0}$ seconds.

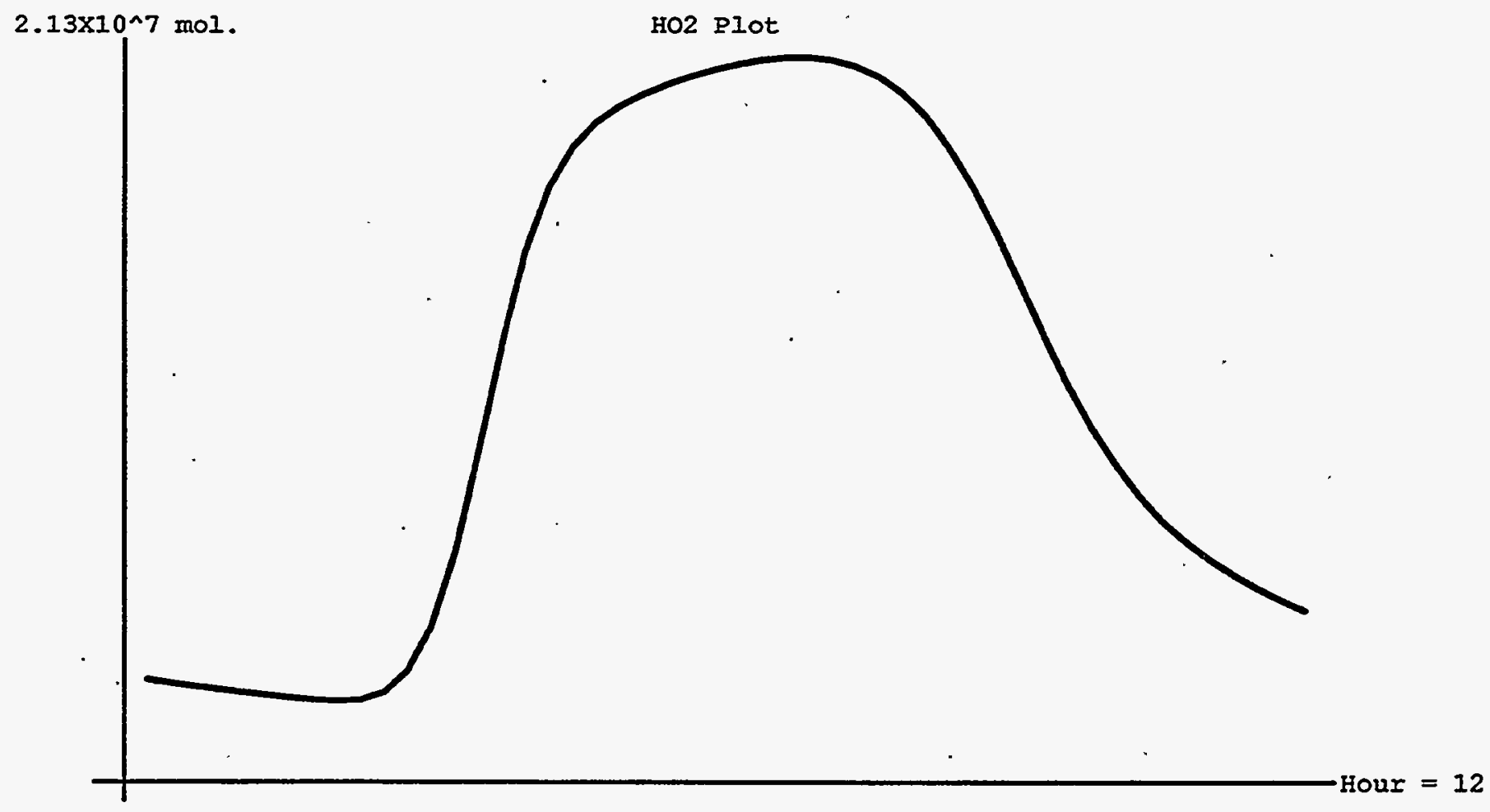




$$
\text { In }
$$




\subsection{The Box Model}

\subsection{Description}

A "box model" [70] isolates the reaction equations in a single spatial zone from a full chemical-radiative-transport model. It uses the same radiative and chemistry routines found in the full models $[36,43,72,75]$, but with no advection or diffusion. In this section, a set of twenty chemical species is used in addition to two that are assumed to be in equilibrium [3, Appendix]. Temperatures and pressures are from U.S. Standard Atmospheres (1976), as is the radiation field. The radiation field uses clear sky, a ground albedo of 0.1 , with only Rayleigh Scattering, and no aerosol/cloud chemistry. There are 47 thermal reactions and 21 photolytic reactions [3, Appendix].

The model is run for several different points in the atmosphere: equatorial and mid-latitude, tropospheric and stratospheric, Winter and Summer. These tests give a good indication of the behavior of preconditioned time differencing in a full model. The drawback to this model is the proportion of run time that is taken up by the radiative calculations. In a chemical-radiative-transport model, radiative calculations are done for each column of zones, while chemistry is done for each zone. Thus, for a full model, chemistry is the dominant computation. Since the box model is only a single zone, however, the overhead spent in doing the radiative calculations is going to limit the overall speedup seen in the model. A good rule of thumb is to assume a $50 / 50$ split in run time for chemistry and radiative calculations.

\subsection{CHEMSODE}

CHEMSODE [3, Appendix] is a collection of FORTRAN subroutines implementing the successful variant of the algorithm developed on the two simple test case problems described earlier. It uses the Jacobi preconditioned trapezoid rule and only a single iteration of the preconditioner. The initial guess $\left(y^{n+1}\right)^{(0)}$. is taken to be the Jacobi preconditioned backward Euler method. This is detailed in algorithm 1.3. 
The major change is in the estimate of the local truncation error $E^{n+1}$ described in section 1.1.2. Enror control is a significant challenge for these methods. The choices are extreme with little possibility for an "in between" compromise. Previously, an extrapolation method comparing the calculation of two half steps with a single whole step was used. This was fairly accurate but obviously expensive. Here, the local error indicator:

$$
E^{n+1}=\frac{2}{c+1}\left(c y^{n+1}-(1+c) y^{n}+y^{n-1}\right)
$$

with $c=\frac{\left(t_{n}-t_{n-1}\right)}{\left(t_{n+1}-t_{n}\right)}$ is used. This indicator gives an estimate of $\Delta t^{2} y^{\prime \prime}\left(t_{n}\right)+O\left(\Delta t^{3}\right)$, which is a bit conservative, but was used with success in $[4,65,67]$. Given this alternate error estimate, the values for $\left\|E^{n+1}\right\|$ and $\Delta t_{\text {new }}$ are computed in the same manner as in section 1.1.2.

The maximum and minimum stepsizes are 1 hour and 1 second respectively. A non-zero minimum is needed because of the intolerably small stepsizes forced by the approximate error indicator Eq. (18) at start-up. The initial stepsize attempted is the minimum step length. All implementation details may be found in [3, Appendix].

CHEMSODE, the implementation of the second order Jacobi preconditioned method (algorithm 1.3), is compared with the ODEPACK [29] routine LSODE [46]. LSODE is currently used in many chemical-radiative-transport models $[36,43,72,74,75]$ and is representative of the state-of-the-art in the robust implementation of implicit multistep methods for the general ODE problem. LSODE uses a Newton iteration to solve the nonlinear system of equations resulting from the implicit calculation. One of LSODE's major optimizations is that it updates the Jacobian matrix used in the Newton iteration only when there is a convergence failure [46], so that it does not necessarily have to calculate and invert the Jacobian at every step. 


\subsection{Timings}

Six test runs are performed using a cross section of points in the atmosphere. The start time (the calendar date), temperature, pressure, and latitude variables are varied but the initial conditions in the model remain constant. The temperature and pressure values are obtained from the U.S. standard atmosphere and represent the desired altitude. The latitude value affects the radiative transfer calculations. The date (time of year) affects the length of day (depending on the latitude) and therefore affects the diurnal cycle and all radiative transfer calculations. Even though initial conditions are the same for all runs, changing latitude, pressure, temperature, and start date provides a good first approximation to the desired atmospheric locations. Table XII summarizes the parameters for all tests.

TABLE XII. Summary of parameters used for all box model tests for the comparison of .

CHEMSODE and LSODE.

\begin{tabular}{l|lll}
\hline Test \# & Altitude & Latitude & Date \\
\hline Test 1 & $26 \mathrm{~km}$ & 5 deg. N. & July 1 \\
Test 2 & $4 \mathrm{~km}$ & $30 \mathrm{deg} . \mathrm{N}$. & July 1 \\
Test 3 & $20 \mathrm{~km}$ & 30 deg. N. & July 1 \\
Test 4 & $40 \mathrm{~km}$. & 30 deg. N. & July 1 \\
Test 5 & $20 \mathrm{~km}$. & 30 deg. N. & Jan. 2 \\
Test 6 & $40 \mathrm{~km}$ & 30 deg. N. & Jan. 2
\end{tabular}

All runs begin at noon local time and integrate forward 24 hours. Table XIII shows CPU times for all tests and the speedup obtained by CHEMSODE. In each case, CHEMSODE is the winner, averaging approximately a $20 \%$ speedup over LSODE. In the next section, the numerical results obtained by CHEMSODE are compared with those of LSODE.

TABLE XIII. CPU times for CHEMSODE and LSODE on all test problems. Speedup is defined as LSODE's CPU time divided by CHEMSODE's CPU time.

\begin{tabular}{l|lll}
\hline Test \# & LSODE & CHEMSODE & Speedup \\
\hline Test 1 & 427.6 seconds & 328.1 seconds & 1.30 \\
Test 2 & 461.4 seconds & 400.7 seconds & 1.15 \\
Test 3 & 460.0 seconds & 344.6 seconds & 1.33 \\
Test 4 & 428.1 seconds & 403.3 seconds & 1.06
\end{tabular}


TABLE XIII. CPU times for CHEMSODE and LSODE on all test problems. Speedup is defined as LSODE's CPU time divided by CHEMSODE's CPU time.

\begin{tabular}{l|lll}
\hline Test \# & LSODE & CHEMSODE & Speedup \\
Test 5 & 449.5 seconds & 384.8 seconds & 1.17 \\
Test 6 & 452.2 seconds & 328.9 seconds & 1.37
\end{tabular}

\subsection{Results}

Identical parameters are used for all test runs. The error control values are set in an attempt to achieve less than one percent global error. Since the parameters passed to the solvers control the local error and not the global error, they must be chosen conservatively. Thus, a relative tolerance of $10^{-4}$ and an absolute tolerance of $2.55 \times 10^{-1}$ are used. The absolute tolerance requests an error of approximately one fourth of a particle, since computations are done in species concentrations. Thus, LSODE is called with RTOL set at $10^{-4}$ while ATOL is set at $2.55 \times 10^{-1}$. The maximum order is set at 2 . CHEMSODE uses the same values but, as previously stated, is forced to take a minimum stepsize of 1.0 , because the local error indicator Eq. (18) forces an intolerably small stepsize at start-up. This minimum step constraint is needed to get things started.

During the comparison runs, CHEMSODE and LSODE are called to integrate forward 15 minutes and record output. They are then "restarted" to integrate forward another $15 \mathrm{~min}$ utes, continuing this process until the full 24-hour run is completed. The term "restart" means that the routines begin each 15 minute part of the run as though it was the initial call. This is in contrast to the concept of a "continue" [46, see "SRCOM"] which implies that the integrator picks up where it left off and continues the computation as though it had never stopped. Restarting the routines is representative of the way in which they would be called in a full model. If the number of gridpoints is at all large, the memory required to facilitate a continue call would clearly be prohibitive in LSODE's case [46]. CHEMSODE's one-step nature makes a restart virtually identical to a continue call. This is also a motivation in choosing the trapezoid rule. 
In almost all cases, the difference between the two results is less than one percent, in terms of the average species concentration over the 24 hour period. This corresponds to two significant digits of accuracy. The only areas of interest are for test 3 and test 5 (which is a wintertime run of test 3 ). In these cases, there is a higher error in a few of the species, virtually confined to the $N O_{x}$ family. The errors stay in the $2-3 \%$ range, but are definitely more noticeable than other cases. Overall, accuracy was very good, particularly with the ozone concentrations. Table XIV summarizes the relative error in the 24 hour average species concentrations for some of the more interesting trace gases. The number of significant digits is again calculated by taking the log of the relative (percent) error (error being the difference between LSODE's and CHEMSODE's results).

TABLE XIV. Summary of CHEMSODE accuracies for all test runs on selected species. Agreement to within machine accuracy is indicated by the entry "exact."

\begin{tabular}{l|llllll}
\hline Test \# & $\mathrm{O}_{3}$ & $\mathrm{CH}_{4}$ & $\mathrm{~N}_{2} \mathrm{O}$ & $\mathrm{NO}$ & $\mathrm{NO}_{2}$ & $\mathrm{NO}_{3}$ \\
\hline Test 1 & 4.03 & exact & exact & 2.29 & 2.38 & 2.58 \\
Test 2 & 4.46 & exact & exact & 2.18 & 2.26 & 2.65 \\
Test 3 & 4.53 & exact & exact & 2.02 & 1.93 & 1.96 \\
Test 4 & 3.86 & 4.20 & exact & 3.28 & 3.48 & 3.13 \\
Test 5 & 4.17 & exact & exact & 1.94 & 1.76 & 2.09 \\
Test 6 & 3.16 & 3.92 & exact & 3.66 & 3.16 & 3.12
\end{tabular}

It is insightful to examine the time evolution of two interesting selected species from these tests. Figure 9 shows the ozone plot from test 1 . CHEMSODE is in good agreement with LSODE in this case, as the difference between the two is always much less than one percent. The characteristic rise in ozone concentration during the daylight hours is seen here. This provides additional confirmation that both solvers are working correctly. Figure 10 shows the plot of $\mathrm{NO}_{3}$ taken from test 3. This is one of the cases where CHEMSODE's daily average differed by more that one percent. Also shown in Figure 10 is a plot of the number of significant digits over time. In Figure 10, one can see where CHEMSODE has its problems in modeling a fast transient species. The initial concentration for this species is zero. As a convenience, the initial number of significant digits is defined to be zero as well. The accuracy quickly climbs to a respectable two digits of accuracy until the seventh hour, when the concentration begins to spike. This is where the first dip in the accuracy 
FIGURE 9. Time evolution of ozone for both CHEMSODE (9b) and LSODE (9a).

$1.51 \times 10^{\wedge}-7 \mathrm{~mol} . / \mathrm{mol}$. of air:

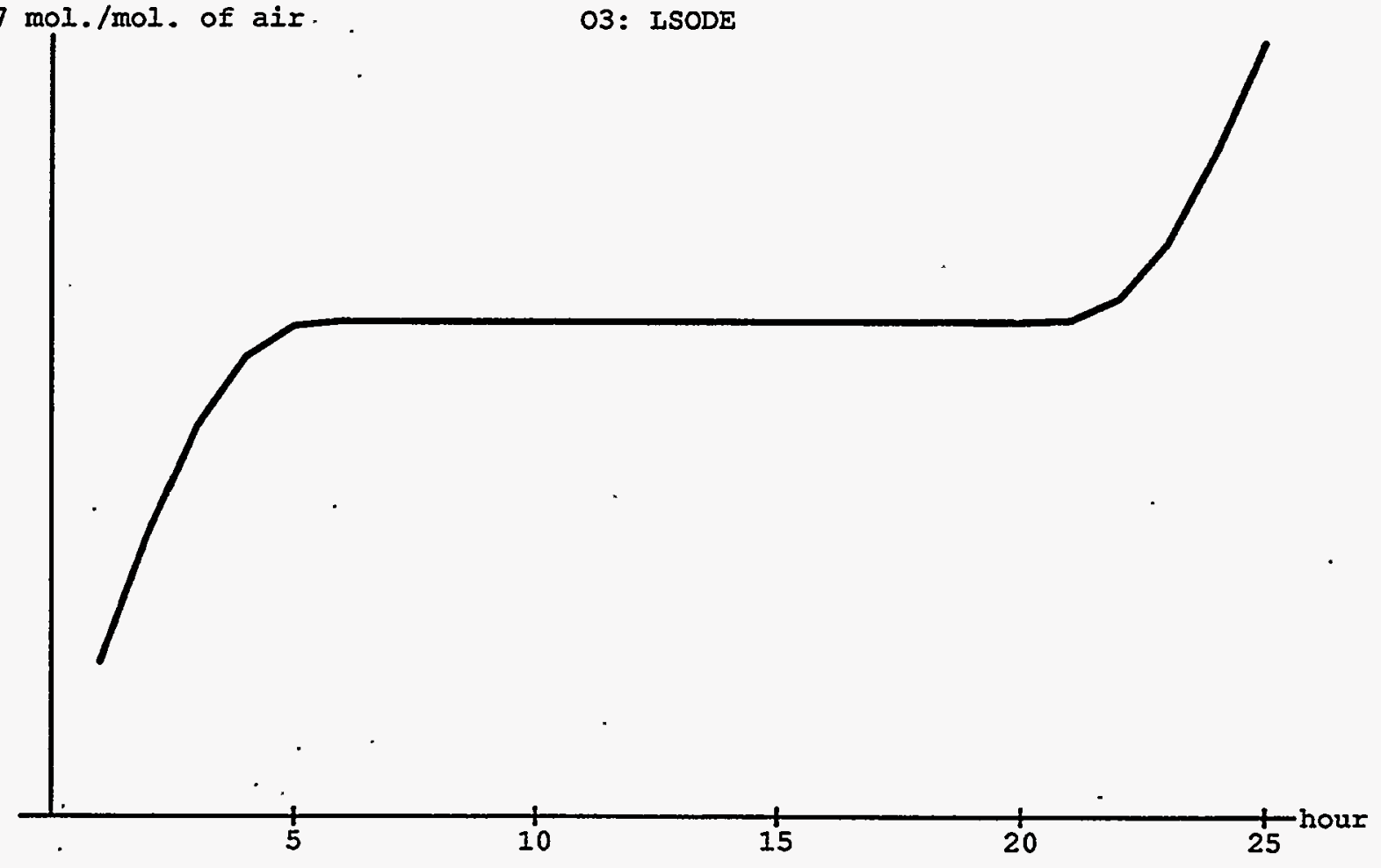


(Figure 9b--Continuation of Figure 9)

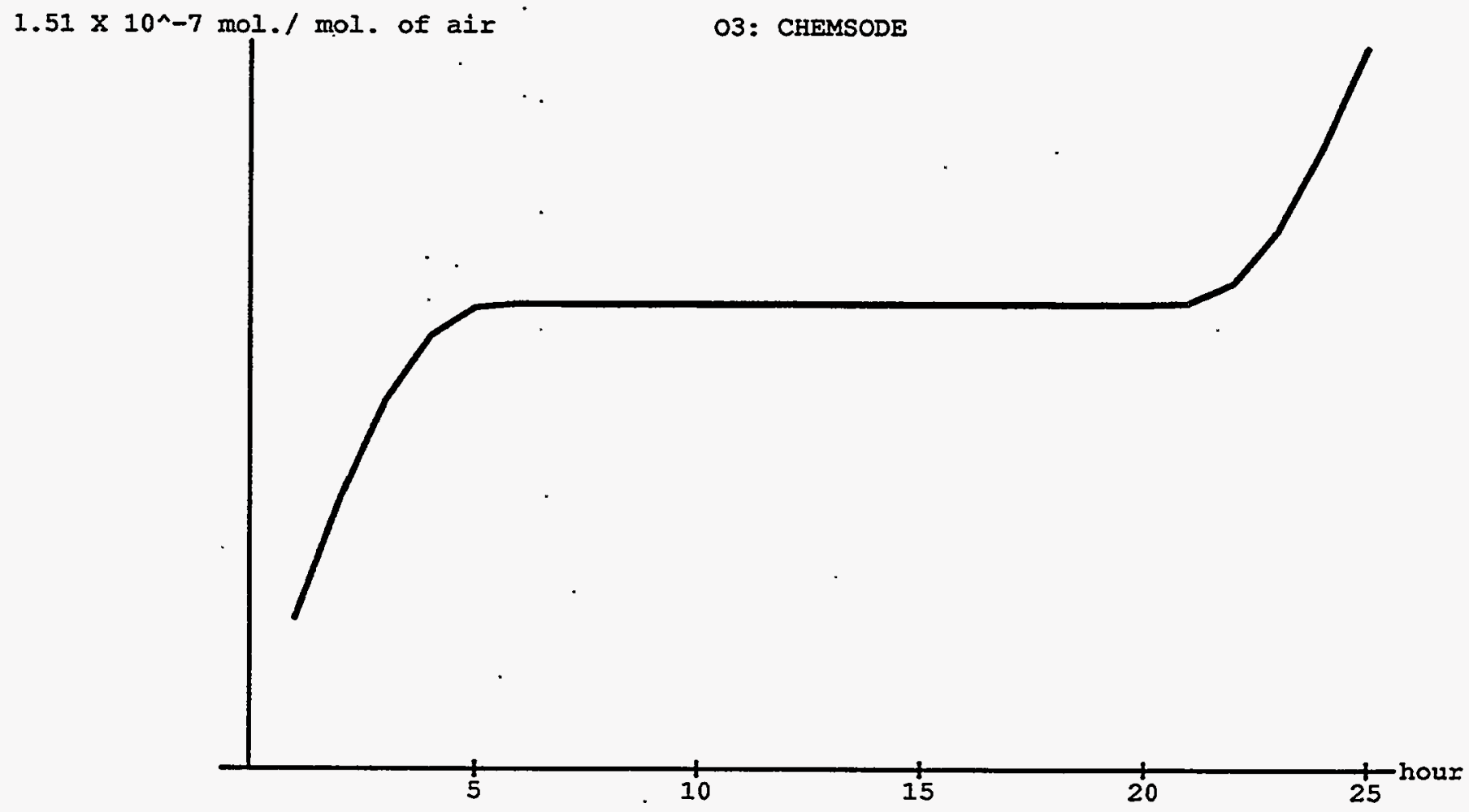


FIGURE 10. Time evolution of NO3 (10a and 10b) along with plot of significant digits vs. time (10c).

$1.72 \times 10^{\wedge}-14 \mathrm{~mol} . / \mathrm{mol}$. of air

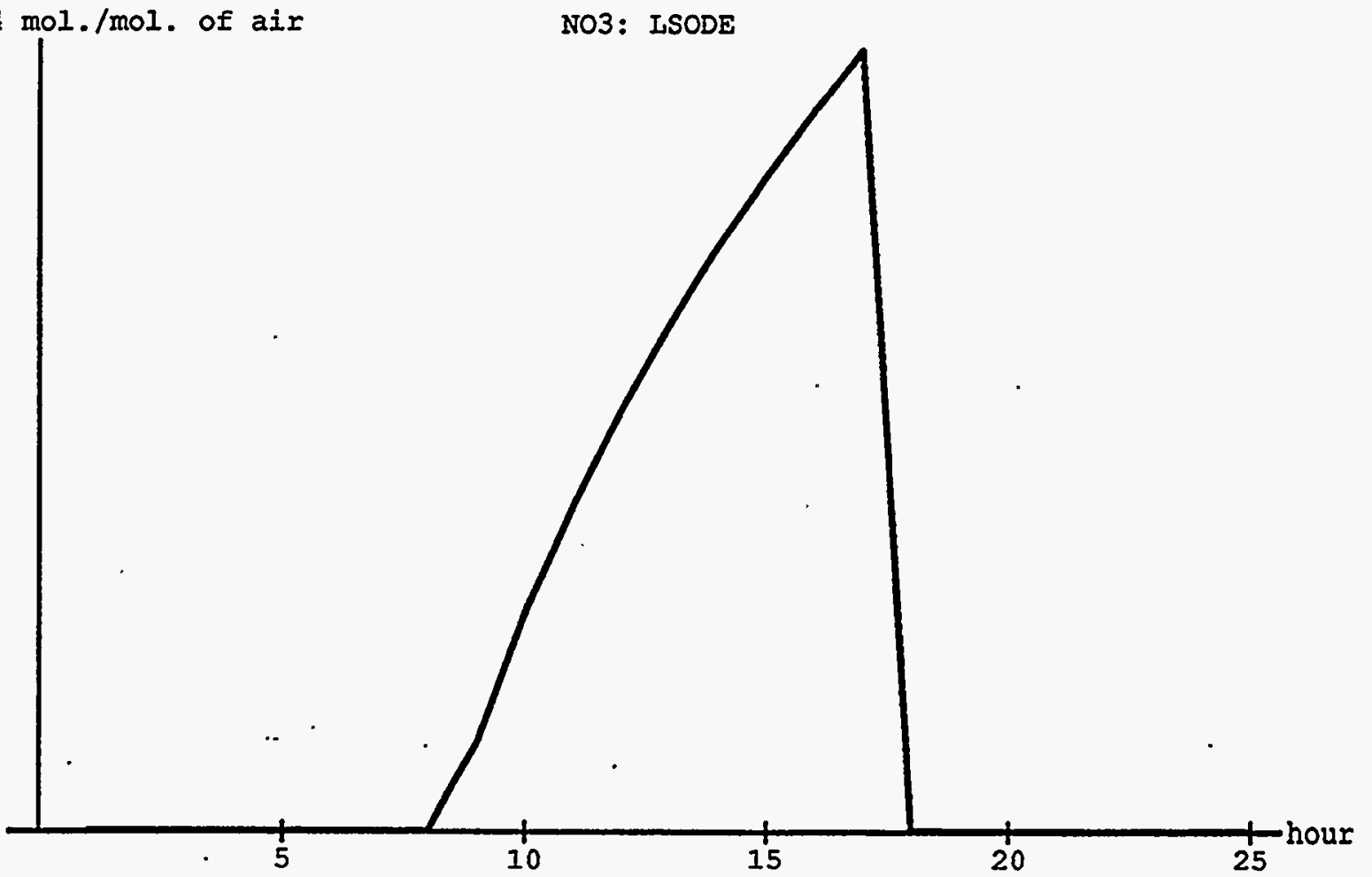

Q 
(Figure 10b--Continuation of Figure 10)

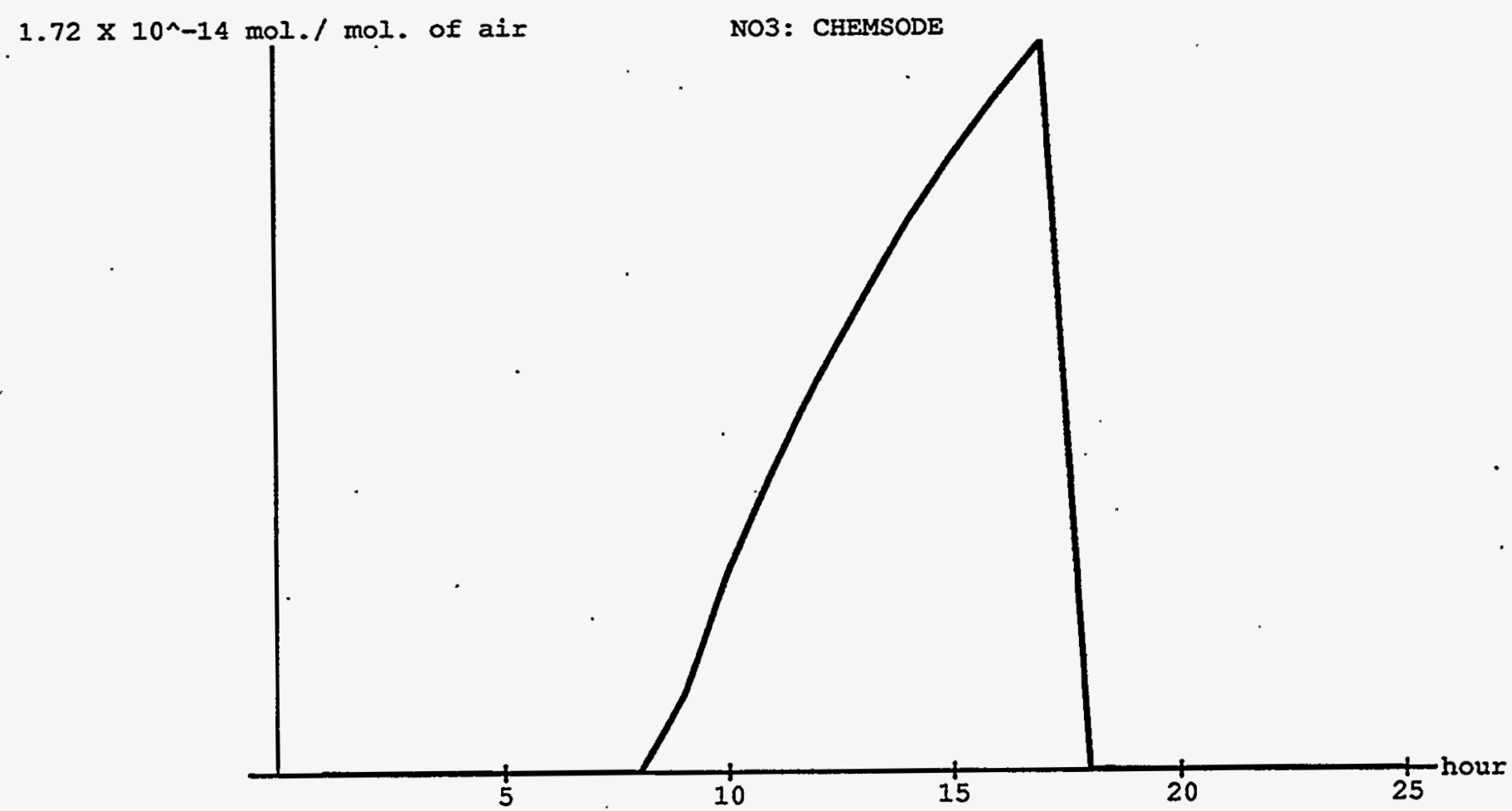


(Figure 10c-Continuation of Figure 10)

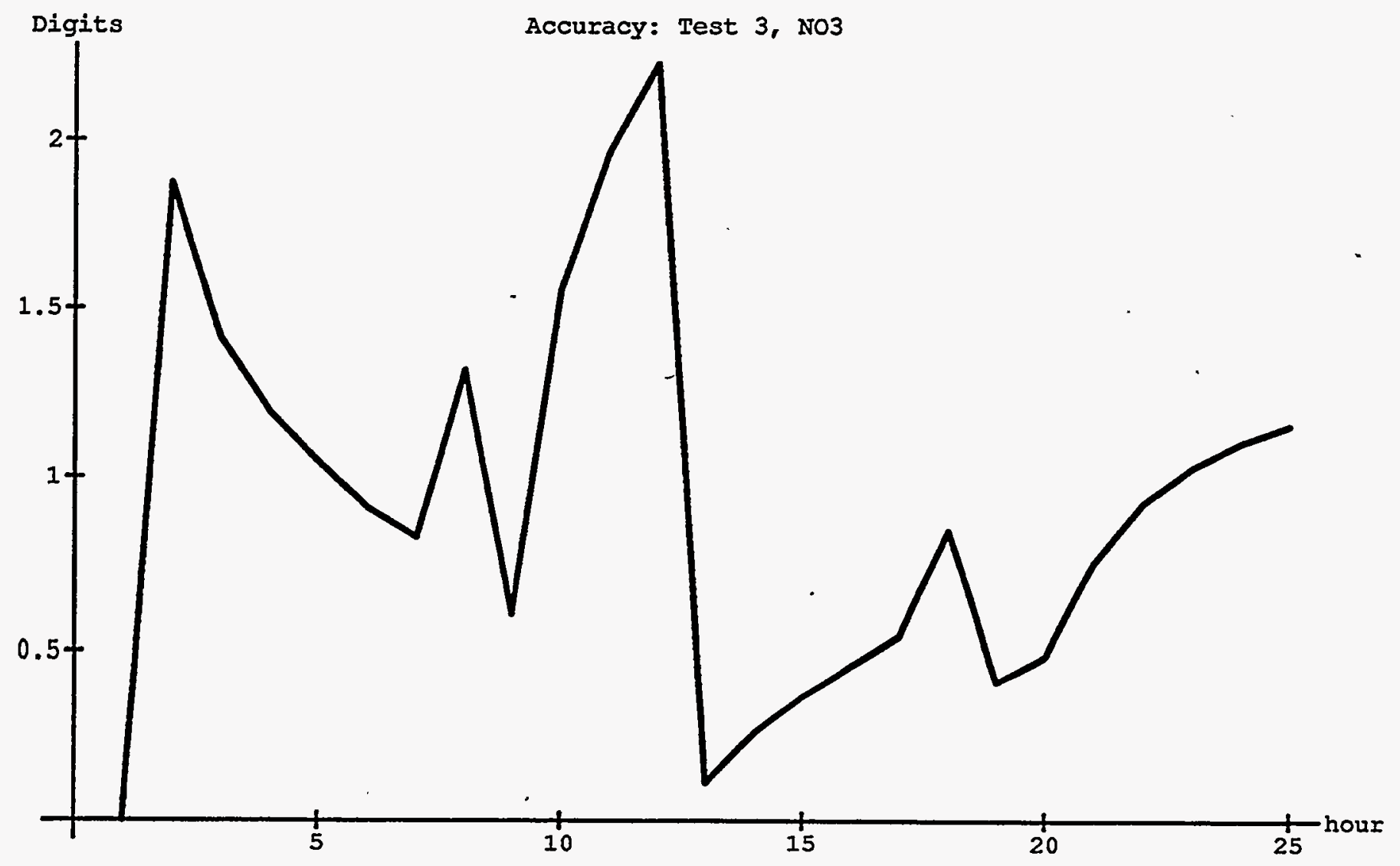


occurs. There is a second dip in accuracy at hour nine, corresponding to the knee in the plot of concentration (seen in 10a and 10b) at hour nine. The major drop in accuracy occurs when the concentration begins to peak out at hour 14 . These problems are caused by the minimum stepsize that must be imposed on CHEMSODE. This is a compromise between reasonable step control, and reasonable accuracy. This study is not interested in the exact modeling of transient behavior (CHEMSODE may not be the solver of choice in that case). In terms of long term average behavior, CHEMSODE is close to the desired two digit accuracy for this species. CHEMSODE's accuracy is therefore sufficient for use in a full model even in the less accurate cases such as this one.

\subsection{Conclusions}

Jacobi and Gauss Seidel preconditioning of the backward Euler and trapezoid rule techniques result in explicitly computable, unconditionally stable methods for atmospheric chemical kinetics problems. During the midday periods, when the reaction rates are nearly constant, Gauss Seidel has a slight advantage over the Jacobi preconditioner, as far as the average step length taken. When the diurnal effect is present, however, this study has shown that the Jacobi method clearly outperforms Gauss Seidel in both the number of steps taken and the CPU time required. It is clear that Jacobi is the preconditioner of choice in a global atmospheric chemical-radiative-transport model. Finally, CHEMSODE's implementation of the Jacobi preconditioned trapezoid rule outperforms the ODEPACK routine LSODE by an average $20 \%$ in a realistic chemistry scenario taken from a full chemical-radiative-transport model. These results lay the foundation for the development of higher order multistep methods that promise to significantly improve the performance of these vital research models. 
CHAPTER 3

\section{Preconditioned Time Differencing in Full CRT Models}

\subsection{Chemical-Radiative-Transport Models}

Atmospheric chemical-radiative-transport (CRT) models are used to investigate the role trace gases have on both chemical and physical processes that determine the distribution of ozone and other trace constituents. A two-dimensional model includes transport in the meridional (latitude) as well as the vertical direction. Therefore, such models offer a more realistic representation of the feedbacks that dynamics can have on the distribution of trace species such as $\mathrm{NO}_{x}, \mathrm{HO}_{x}, \mathrm{Br}_{x}$, and $\mathrm{Cl}_{x}$ than would a 1-D or box model. Since these chemical families control ozone production and loss processes, the role of transport can be extremely important in determining the distribution of ozone in the atmosphere [75]. A three-dimensional model carries this a step further with transport in all directions. In this chapter, CHEMSODE is compared to LSODE in two full CRT models (one 2-D and one 3-D). Tests are performed on both vector and parallel architectures. These tests confirm CHEMSODE's superiority to LSODE for use with CRT models.

\subsection{3-D Model}

The 3-D model will be examined first. Even though it is spatially the more complex of the two models, the chemistry set being examined here is smaller than in the 2-D model. The 
3-D model is in the latter stages of development and has not yet incorporated full chemistry. The high amount of CPU time forces the use of a small number of chemical species at this early stage. An examination of the architectures to be used is presented here.

\subsection{Architectures}

Tests are performed for two different architectures: one high performance vector machine, and one massively parallel machine. In each case, CHEMSODE is shown to be the superior choice. Also shown is the atmospheric CRT problem's suitability for parallel computing.

\subsubsection{CRAY C-90}

The CRAY C-90 consists of 16 vector processors compatible with the CRAY Y-MP design. There are two vector pipes per CPU running on a 4.2 ns clock giving each processor a peak performance of 1 gigaflop. It has four parallel memory ports per CPU, 16 channel adapters per cluster (for $\mathrm{I} / \mathrm{O}$ ), and a memory bandwidth of 245 gigabytes per second. This supports the vector and scalar performance with the highest memory and I/O bandwidth available and results in a very balanced architecture. It is the state-of-the-art in production vector machines.

\subsubsection{Meiko CS-2}

The Meiko CS-2 is a 256-node multiple-instruction multiple-data (MIMD) architecture offering massive parallelism with a choice of processor architecture. Its processing elements are scalar configured for results presented here.

The processing element nodes (PENs) consist of two $90 \mathrm{MHz}$ Sparc chips (capable of 180 Megaflop performance) and a local cached memory system. Communication is accomplished via message passing using a multi-stage switching network constructed from 8 by 8 crosspoint switches. This multi-stage packet switch is a fat tree in which the bandwidth between stages remains constant. The number of network stages is logarithmic in the number of processing element nodes: two stages connect 16 nodes, three connect 64 , etc. The 
longest path between any two PENs in the 256 node system is seven network switches. The network also supports hardware broadcast at full bandwidth and low latency bulk synchronization.

\subsection{IMPACT}

IMPACT, the 3-D CRT model used in this study, is based on the operator splitting method, where each of the primary operators (advection, diffusion, convective mixing, photolysis, and chemistry (including sources)) is dealt with in an independent fashion. Advection in all three directions is currently treated using a second order van Leer method (an upstream-biased monotonic grid point scheme) $[2,30,64]$. The upstream nature of this method reduces phase errors to a minimum and the monotonicity control eliminates the need for a filling algorithm and the severe problems that would arise with negative values of the chemical species concentrations. Diffusion is currently included using a very simple constant coefficient multiplied by a grid-based second derivative [38].

Photolysis implementation allows simulations using either complete diurnal calculations or diurnally averaged reaction rate coefficients. In this study, complete diurnal calculations will be used. The photolytic loss rate constants are calculated by integrating the product of absorption coefficient, quantum yield, and solar flux over wavelength ( $175 \mathrm{~nm}$ to $760 \mathrm{~nm}$ ). To capture the spectral detail needed for photodissociation calculations, the two-stream, multiple-layer, UV-visible model uses 126 wavelength bins between $175 \mathrm{~nm}$ and $735 \mathrm{~nm}$. The scattering of energy from the direct solar beam within each individual layer is treated using the delta-eddington algorithm [34]. The scattering of diffuse radiation (i.e. previously scattered radiation) from each individual layer is modeled using the simpler SaganPollack algorithm [50]. Both algorithms allow inclusion of the bulk optical properties of clouds and aerosols. Finally, the adding method is used to calculate irradiances throughout the vertically inhomogeneous atmosphere. The absorption cross sections and quantum yields include temperature and pressure dependence where appropriate and available.

IMPACT was implemented onto the parallel architecture using a two-dimensional domain decomposition paradigm where the horizontal direction is divided into sections and each 
section is then given to a separate processor. This shall produce a slight load imbalance when the model is run on a small number of nodes (more detail later). The advantage to this type of domain decomposition is that it leaves the vertical extent of the domain intact while resulting in a number of smaller blocks of grid zones. The paradigm works extremely well for CRT models which use the vast majority of time to evaluate photolysis rates and solve the chemical equations. Photolysis is a column calculation and chemistry is a local calculation, thus both calculations are done within the node and require no interprocessor communication creating a scalable, parallelized code.

IMPACT can be run either in an interactively coupled mode or stand-alone (off line) using externally obtained meteorological fields (for example, derived from a general circulation model or from assimilated data sets). For the calculations performed in this study, done off line, the use of data assimilated meteorological fields (winds, temperature, etc.) from the Data Assimilation Program at NASA Goddard has been adopted.

\subsubsection{Chemistry}

Due to the large amount of CPU time needed at this early stage, a limited chemistry scenario will be used. There are eight chemically reacting species used in these tests, $\left(\mathrm{O}_{3}\right.$, $\mathrm{H}_{2} \mathrm{O}, \mathrm{OH}, \mathrm{HO}_{2}, \mathrm{H}_{2} \mathrm{O}_{2}, \mathrm{CH}_{4}, \mathrm{CH}_{2} \mathrm{O}, \mathrm{CO}$ ), modeled by 15 thermal reactions and 6 photolytic reactions (listed in Appendix B).

\subsubsection{Timings}

IMPACT was started at noon on sidereal day two. The model timesteps were five minutes long in order to keep the local error of the advection scheme under the one percent level. Within the model, LSODE was called with RTOL set at $10^{-4}$ (in the same spirit as with the box model calculations) and ATOL set at $10^{-20}$. ATOL is set in this way so that the local error does not exceed machine roundoff (here, calculations use mixing ratios). CHEMSODE used the same parameters, with the exception of a minimum stepsize of one second, as was used in the box model comparison. 
During the runs, CHEMSODE and LSODE are called to integrate forward 5 minutes and record output. At the next model step, they are "restarted" to integrate forward another 5 minutes, continuing this process until the full run is completed. As in the previous chapter, the term "restart" means that the routines begin each 5 minute part of the run as though it was the initial call. Restarting the routines is necessary in LSODE's case due to the prohibitive amount of memory required to facilitate a continue for each zone in the model [4].

Initial runs are performed on the CRAY C-90. Code is written to allow the compiler to vectorize when possible. No special attempts were made to increase vectorization as in [33] however, so that accurate comparisons between the preconditioned method and the backward differentiation formulas used by LSODE were attained.

In table I, C-90 runtimes are shown for IMPACT using both CHEMSODE and LSODE. CHEMSODE is superior for both one and ten model steps. Interesting to note is that CHEMSODE is more favorable on longer runs. For one model step, IMPACT runs $39 \mathrm{per}-$ cent faster using CHEMSODE. For ten model steps, CHEMSODE makes the model 85 percent faster. The reason for this behavior is the necessity to restart LSODE at each call. This forces LSODE to immediately calculate and invert a Jacobian matrix, something it may not have to do on a continue call. CHEMSODE's one step explicit nature makes restarts a non-issue, since no Jacobian matrix is necessary. Thus, one shall characteristically see the speedup grow for longer runs.

TABLE I. Timings for one and ten time steps: CRAY C-90

\begin{tabular}{llll}
\hline \# of steps & CHEMSODE & LSODE & speedup \\
1 & 1947 seconds & 2699 seconds & 1.39 \\
10 & 7191 seconds & 13306 seconds & 1.85
\end{tabular}

Runs on the Meiko CS-2 were performed with no special attempts at load balancing. Sunrises pose a challenge to the chemistry integrator, since species concentrations tend to change quite sharply at the moment of sunrise [4]. Ideally, one would like the number of spatial zones undergoing a sunrise to be evenly distributed among the processing nodes. Although conceptually easy, this is very difficult to implement. Thus, the spatial zones . were partitioned according to atmospheric location with no special attention given to the 
section of the atmosphere undergoing sunrise. Thus, the effects of slight load imbalance will be seen when the number of nodes is small.

Table II lists runtimes for the Meiko CS-2. Each row shows the runtime for both CHEMSODE and LSODE for the number of nodes given in the first column. Enclosed in parentheses are the runtimes for one and five model steps. The time for one model step is subtracted from the time for five model steps with the resulting quantity divided by four. This gives an average runtime per step without the overhead of start-up.

Again, CHEMSODE is faster in each instance, although only moderately so. An interesting phenomenon is that the average runtime per model step is larger than the time for the initial model step when using 26 nodes or less. This is caused by the load imbalance of having the majority of zones seeing a sunrise assigned to a small number of nodes. Running with a larger number of nodes breaks the region up a little more and brings the overall runtime down.

TABLE II. Timings for Meiko CS-2: (one step, five steps) avg. time per step in seconds.

\begin{tabular}{lll}
\hline \# of nodes & CHEMSODE & LSODE \\
5 & $(2601,14600) 3000$ & $(2879,16523) 3411$ \\
10 & $(1305,7609) 1576$ & $(1374,8216) 1711$ \\
17 & $(786,4189) 851$ & $(867,4675) 952$ \\
26 & $(550,2921) 593$ & $(607,3112) 626$ \\
37 & $(429,2091) 416$ & $(458,2177) 430$ \\
50 & $(349,1547) 300$ & $(433,1711) 320$ \\
65 & $(294,1224) 233$ & $(301,1322) 255$ \\
82 & $(260,971) 178$ & $(264,1074) 203$
\end{tabular}

Another interesting note is how well suited the atmospheric problem is to parallel computing. Note that the model's performance scales almost perfectly with the number of nodes. Performance is defined to be the inverse of the runtime and the speedup is defined to be 15,000 times performance. Speedup is defined in this way so that the speedup for five . nodes (which is the baseline for comparison) is five. Figure 1 shows the speedup versus the number of nodes for the CHEMSODE runs. It is very nearly a perfect linear speedup. 


$$
\bar{Z}
$$


An extended runtime comparison is presented in table III. The model was run for 144 model timesteps or twelve hours of simulation. Again CHEMSODE's performance increases for the longer run. Although it was only 10 to 40 percent faster for the short runs in table II, it shows twice the performance of LSODE for the extended run. This is again a result of LSODE having to restart at each model step.

TABLE M. Timings for extended run on Meiko CS-2: 144 time steps (0.5 days)

$\begin{array}{llll}\text { \# of nodes } & \text { CHEMSODE } & \text { LSODE } & \text { Speedup } \\ 65 & 4372 \text { seconds } & 9233 \text { seconds } & 2.11\end{array}$

\subsubsection{Results}

The difference between the numerical results obtained by LSODE and CHEMSODE is examined for a run of ten model steps (nearly an hour of simulation). Figures 2 through 7 show the relative error (error being defined as the difference between LSODE's and CHEMSODE's computed values) for ozone at several different slices in the atmosphere. Figure 2 shows the relative error at approximately $40 \mathrm{~km}$ (high stratosphere). At this altitude, the relative difference between CHEMSODE and LSODE is much less than one percent. The area of greatest discrepancy is the small area over Antarctica near 60 degrees longitude. Here, the accuracy flirts with the $0.01 \%$ error level only in a few localized spots. Overall, accuracy is acceptable, however. As the model gets into the mid-stratosphere (Figure 3), the accuracy improves noticeably. In Figure 3, the calculations agree to a one thousandth of a percent or better everywhere. This is the section of the atmosphere where the chemical mechanism in this model is most appropriate and the results are predictably very good. Interesting to note is that the error begins to show a correlation with latitude.

Going further into the middle stratosphere (Figure 4), CHEMSODE continues to show good agreement with LSODE and a somewhat stronger correlation between latitude and the discrepancy between the two solvers. Figures 5, 6, and 7 show low stratospheric error plots and continue the trend of good accuracy with a strong correlation between accuracy and latitude: At this stage, IMPACT is not equipped with a large enough chemical mechanism to model the troposphere in a meaningful way. This study, therefore, only examines 
the results of IMPACT's stratospheric calculations. Predictably, CHEMSODE is accurate to at least a thousandth of one percent almost everywhere. Thus, accuracy is well within acceptable tolerances.

Figures $8,9,10$ and 11 show the computational results of extended runs in the upper atmosphere. Figure 8 shows the numerical results obtained after ten model steps for ozone in the middle stratosphere. There is not much change in the makeup of this section of the atmosphere after such a short run. In contrast, Figure 9 shows the ozone results of the same run at a high stratospheric location. The activity in this region is more lively, with noticeable changes in concentration after only ten model steps.

Figure 10 shows the ozone results of an extended run of 200 model steps in the middle stratospheric region (corresponding to Figure 8). In contrast to Figure 8, a fair amount of change has occurred by this point in the simulation. The results seem to be dominated by the advective process. Figure 11 shows ozone from a run of 200 steps in the region corresponding to Figure 9. It also shows a great deal of change, with results that seem to be dominated by the chemical process.

From the preceding figures, we can conclude that CHEMSODE is meeting the accuracy requirement quite well at a reasonable savings in CPU time. This study has now shown CHEMSODE to be the superior choice for a full CRT model. The solver must now be run on a larger chemistry set to confirm these results; this will require the use of a two dimensional model. Even with the speedup obtained by this new solver, the CPU time required for a larger chemistry in 3-D could be significant. 2-D tests will be run in the next section. 
FIGURE 2. CHEMSODE's relative error after 10 model steps at reference altitude 16.

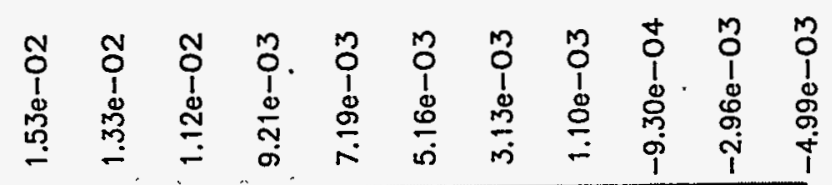

(x)

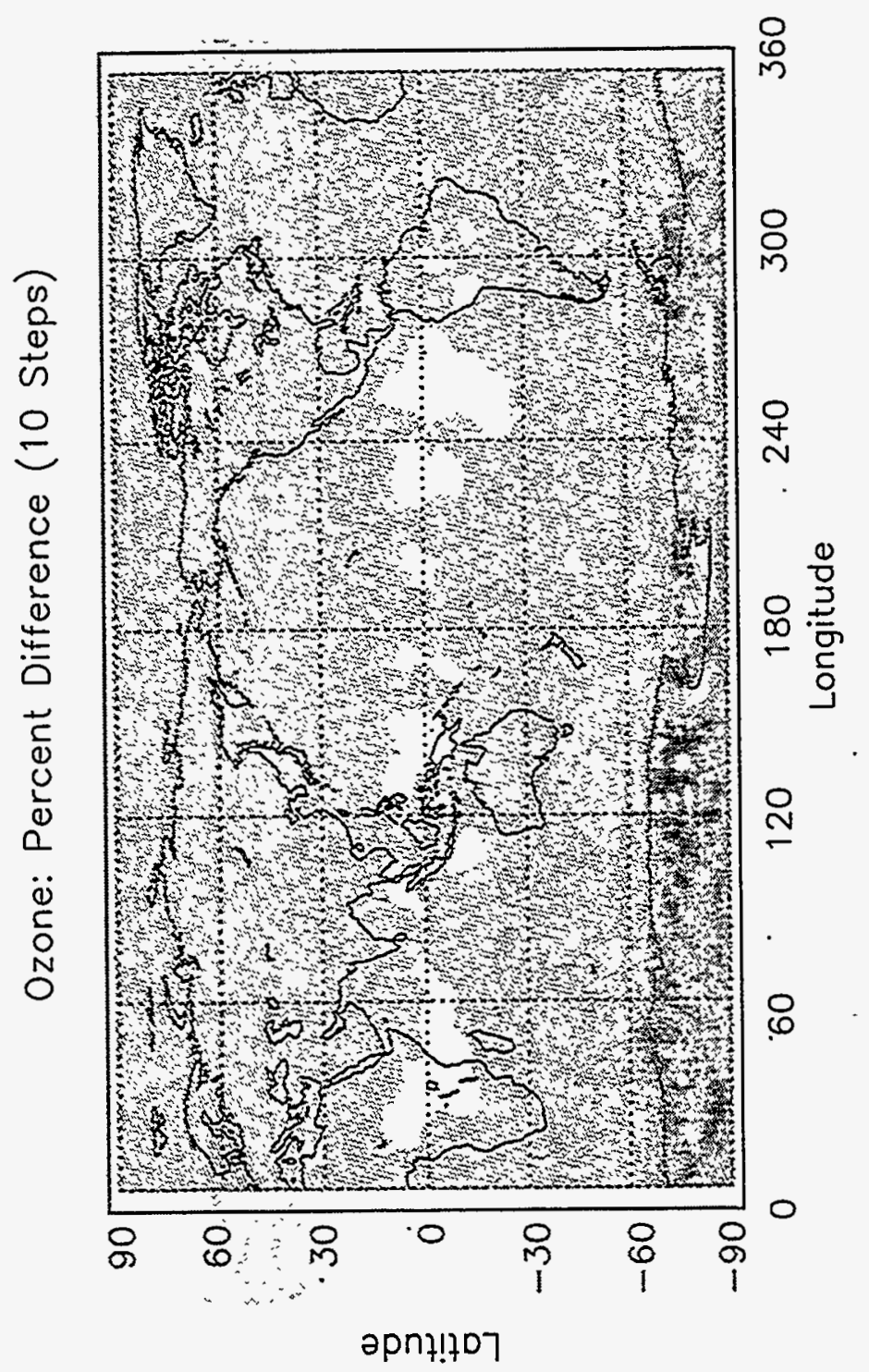

$\frac{0}{11}$
$\frac{0}{2}$
$\frac{⿱}{5}$
$\frac{1}{2}$ 
FIGURE 3. CHEMSODE's relative error after 10 model steps at reference altitude 15.

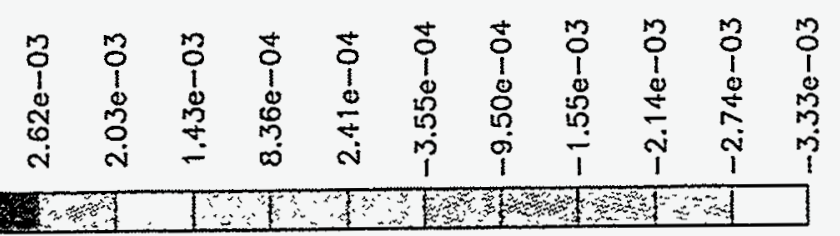

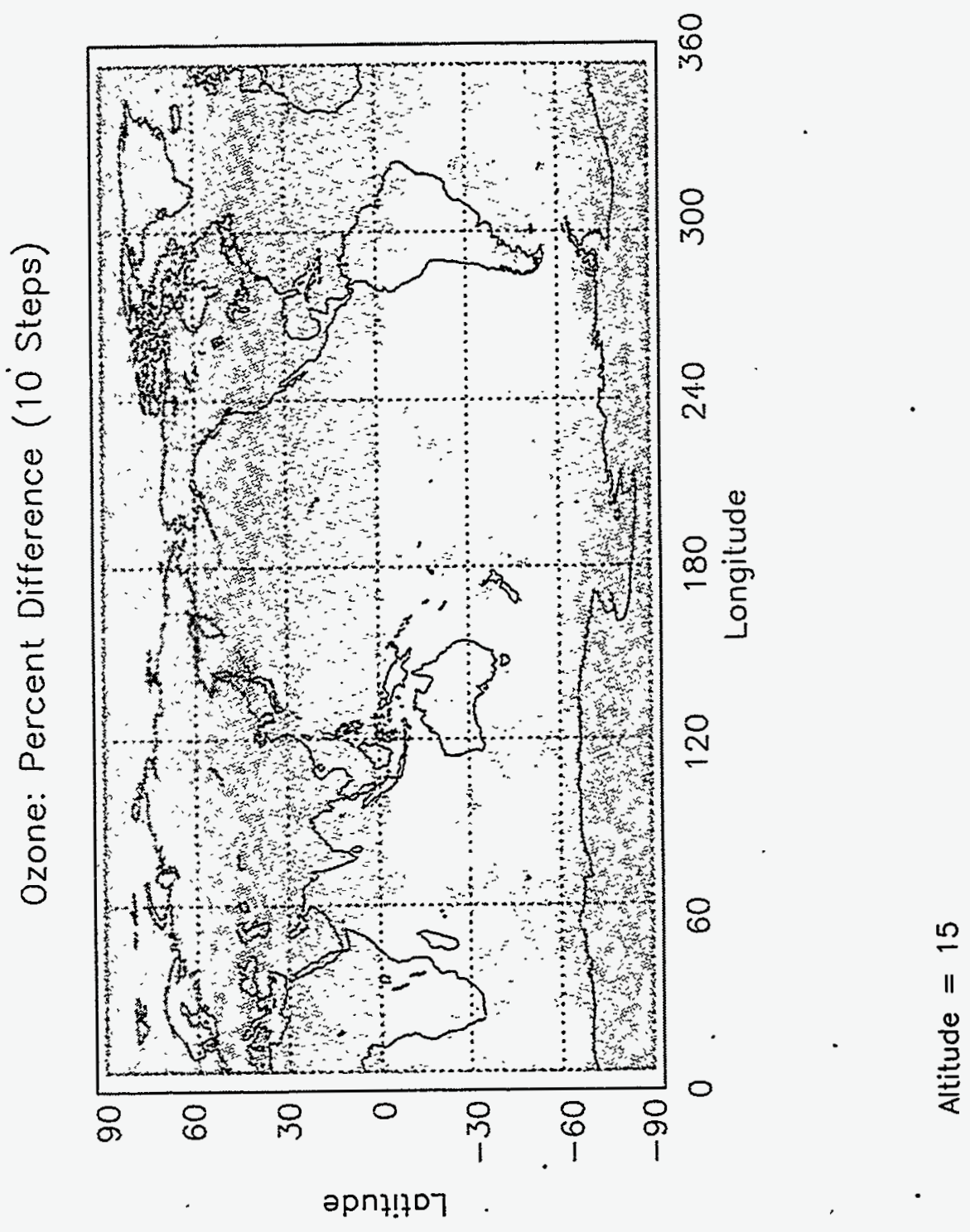


FIGURE 4. CHEMSODE's relative error after 10 model steps at reference altitude 14.

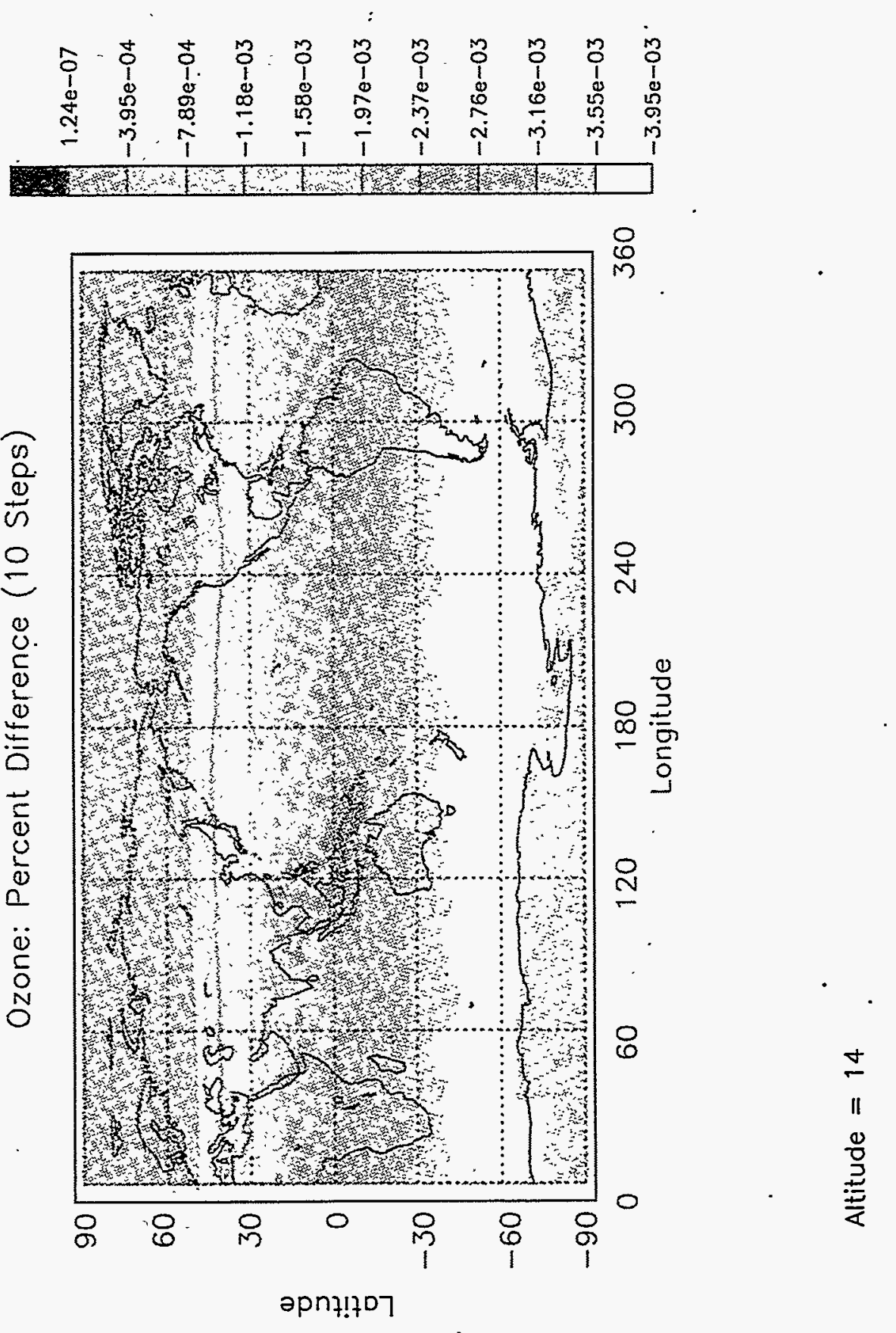


FIGURE 5. CHEMSODE's relative error after 10 model steps at reference altitude 13.

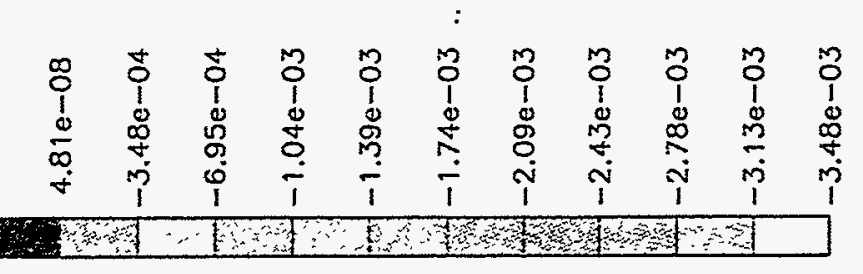

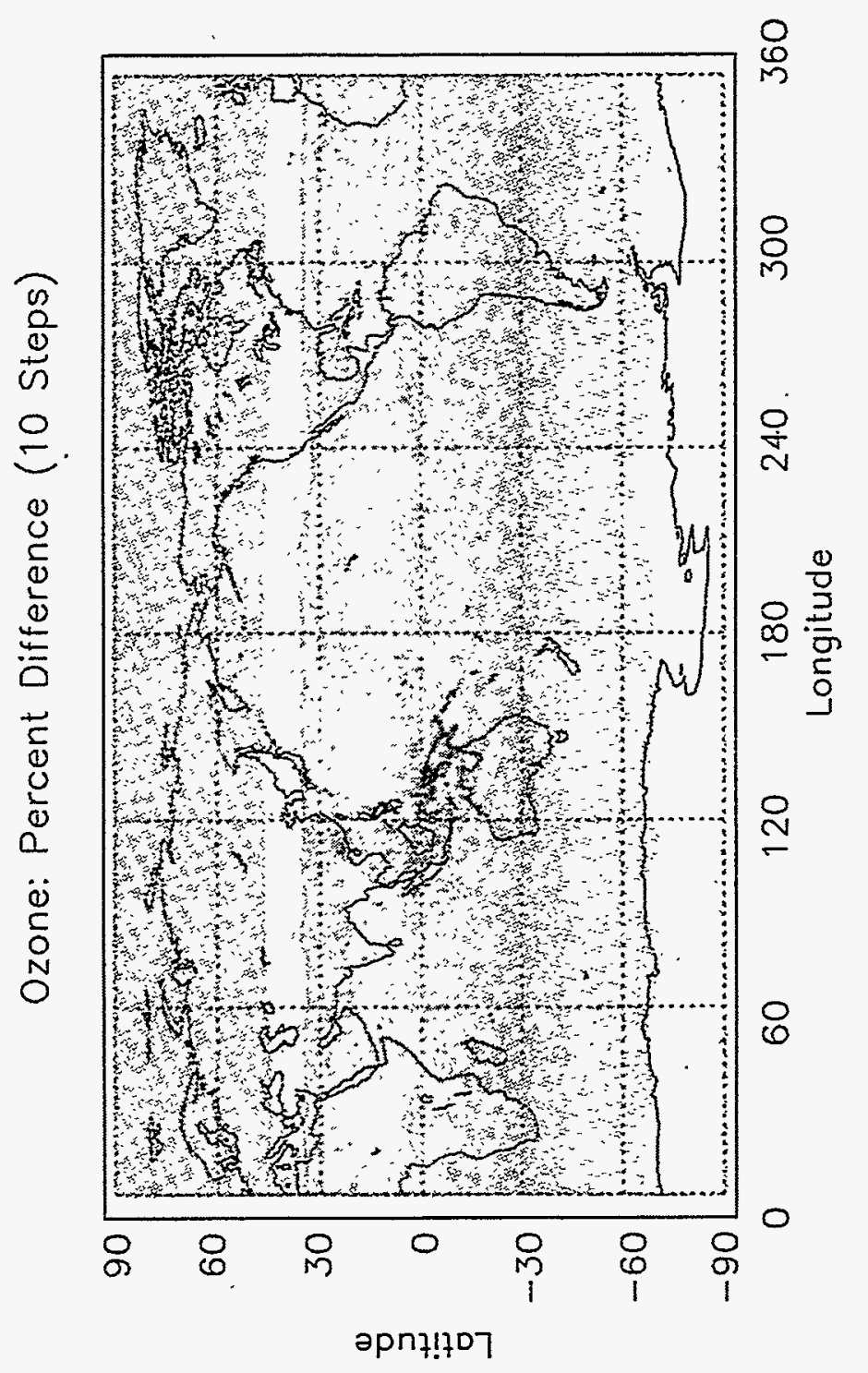

$m$
11
$\frac{2}{2}$
$\frac{2}{2}$ 
FIGURE 6. CHEMSODE's relative error after 10 model steps at reference altitude 12.
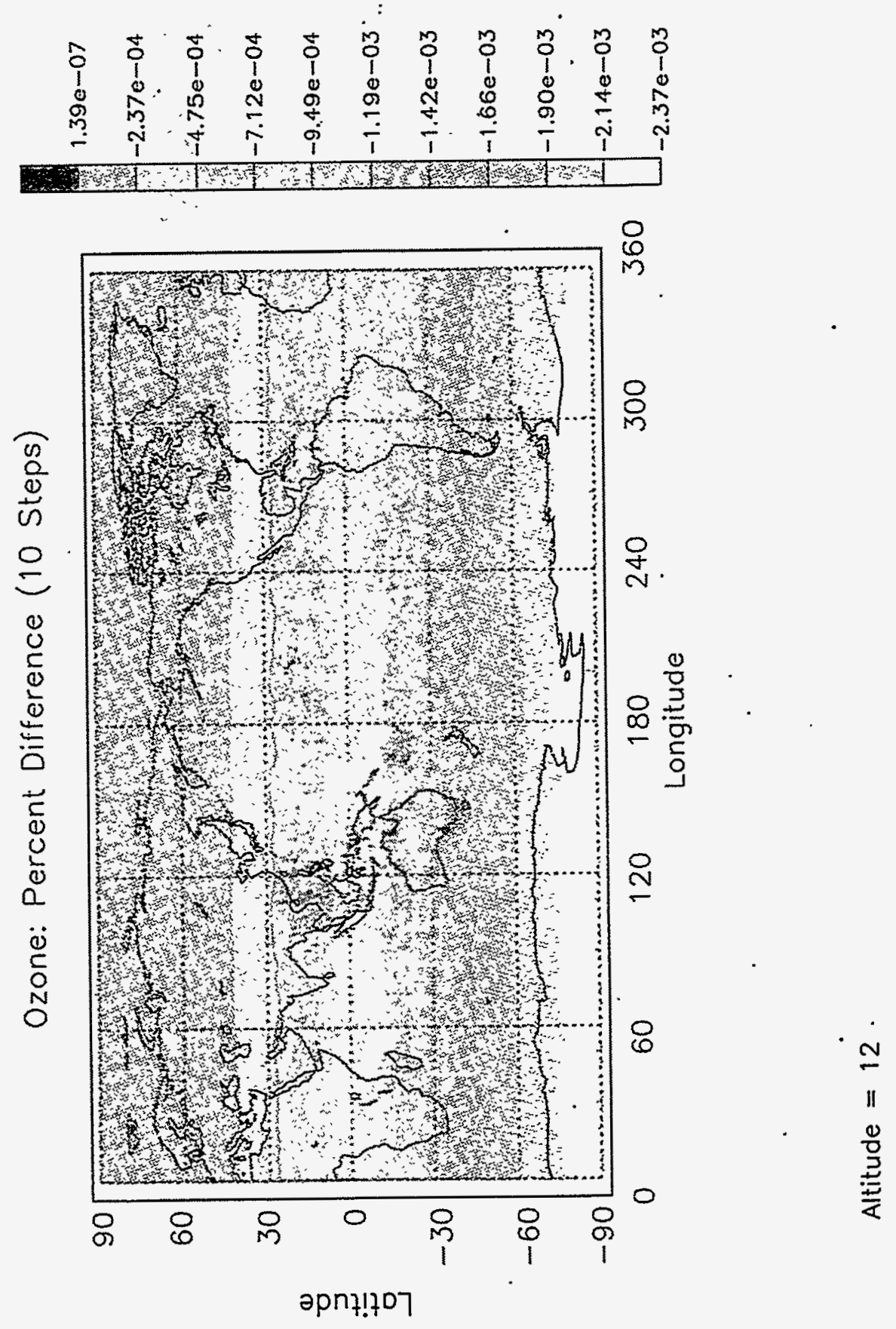
FIGURE 7. CHEMSODE's relative error after 10 model steps at reference altitude 11.

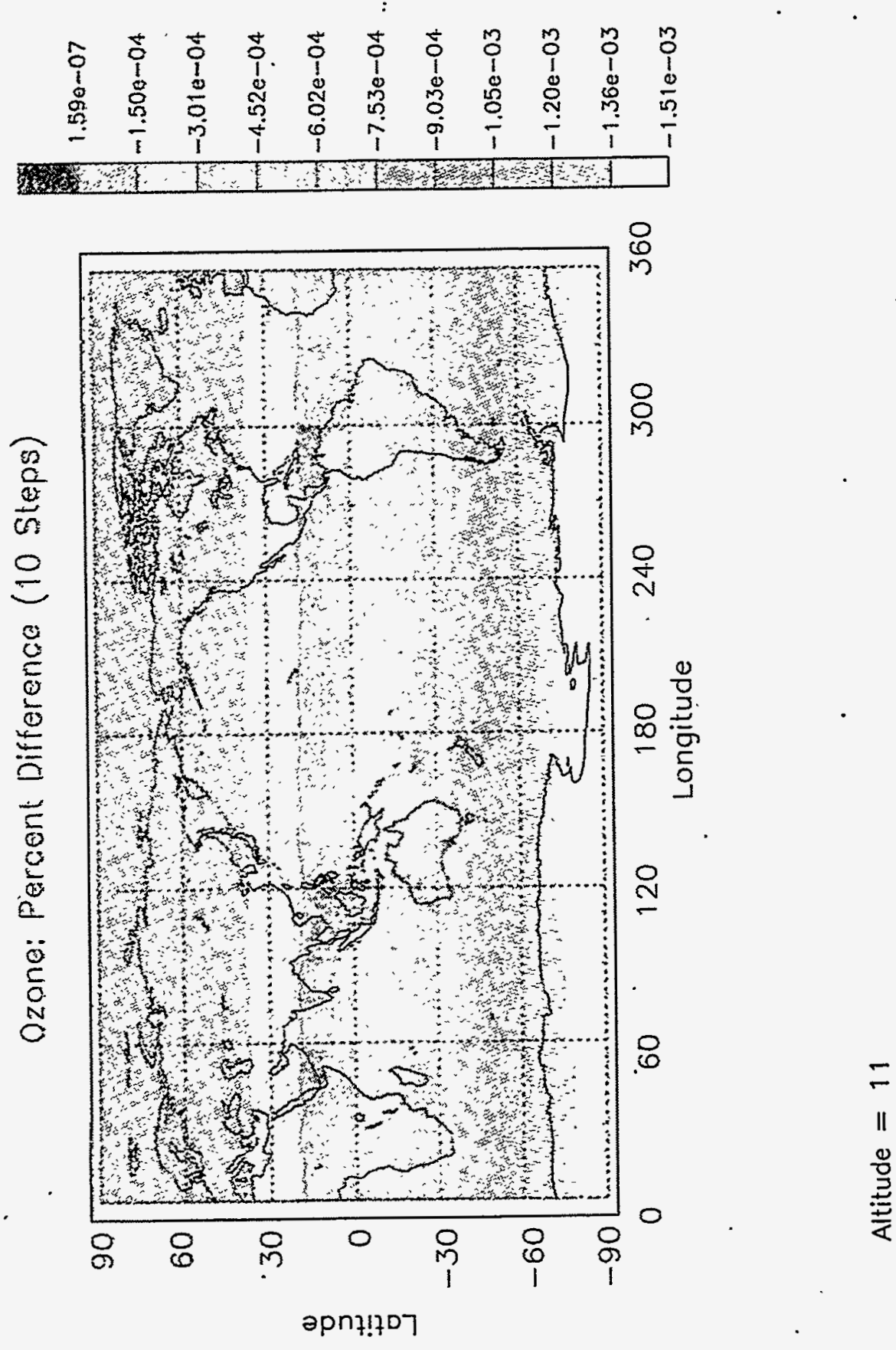




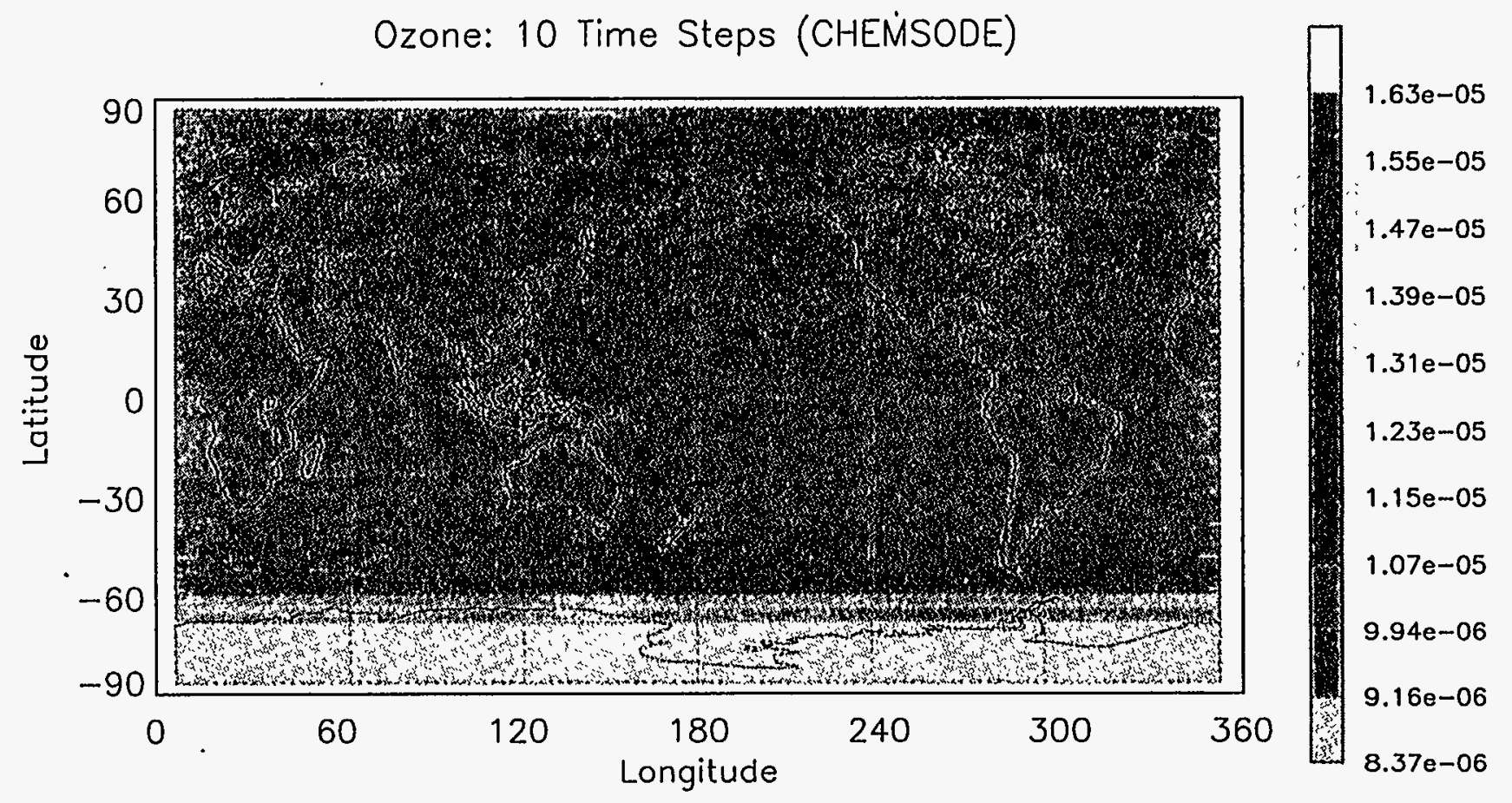

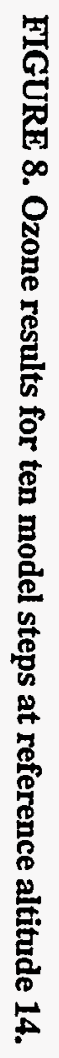

Altitude $=14$ 
FIGURE 9. Ozone results for ten model steps at reference altitude 16.

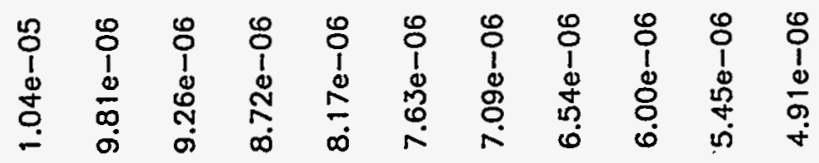

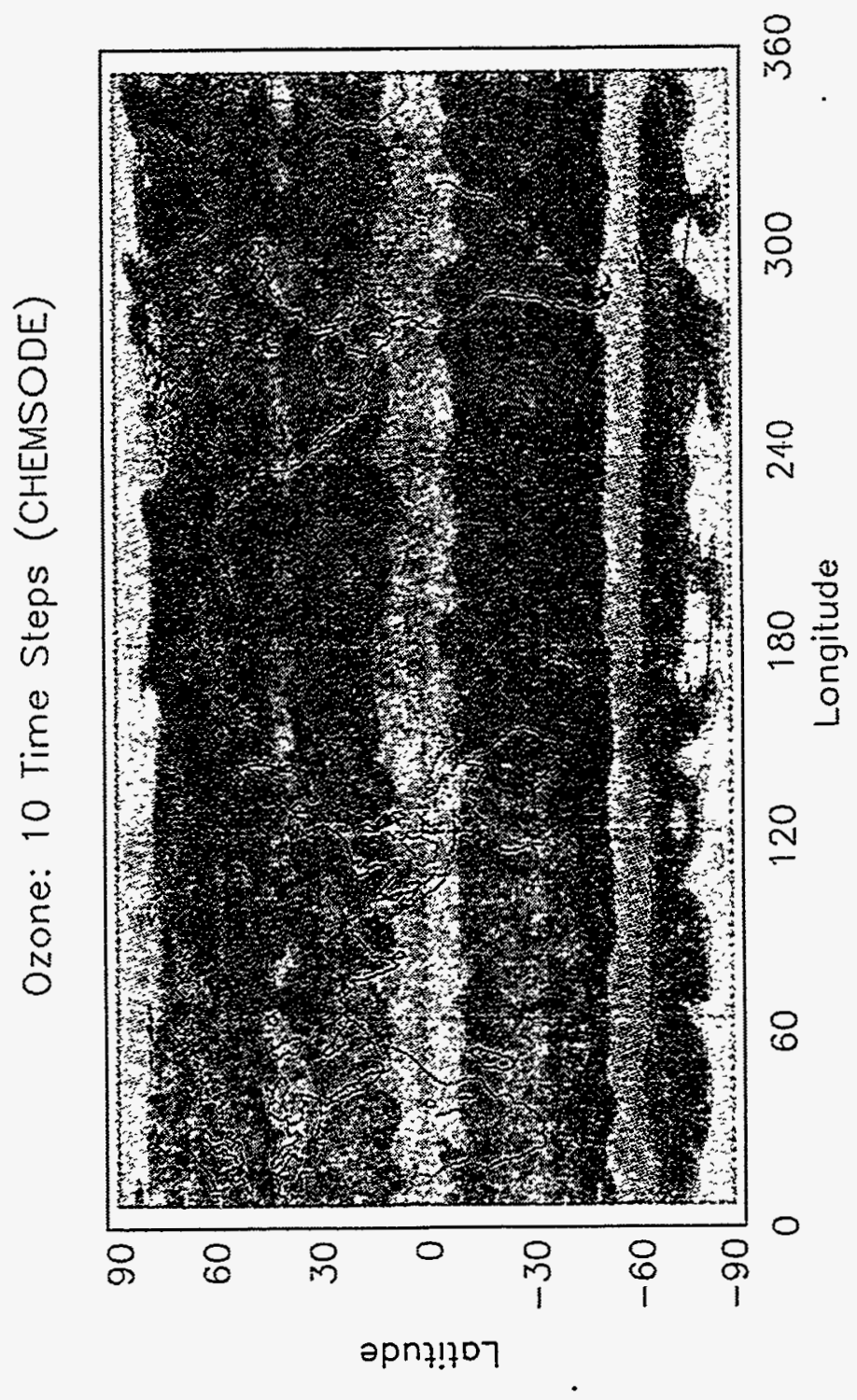

2
11
0
$\frac{0}{2}$
$\frac{ \pm}{ \pm}$ 
FIGURE 10. Ozone results for two hundred model steps at reference altitude 14.

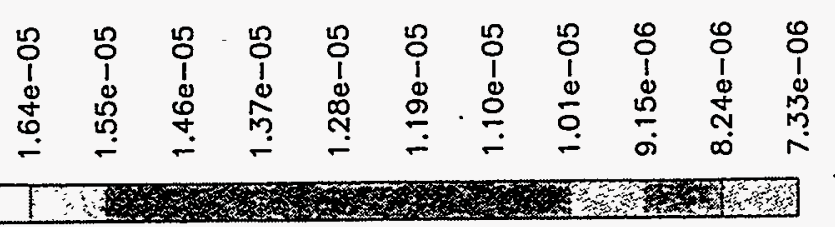

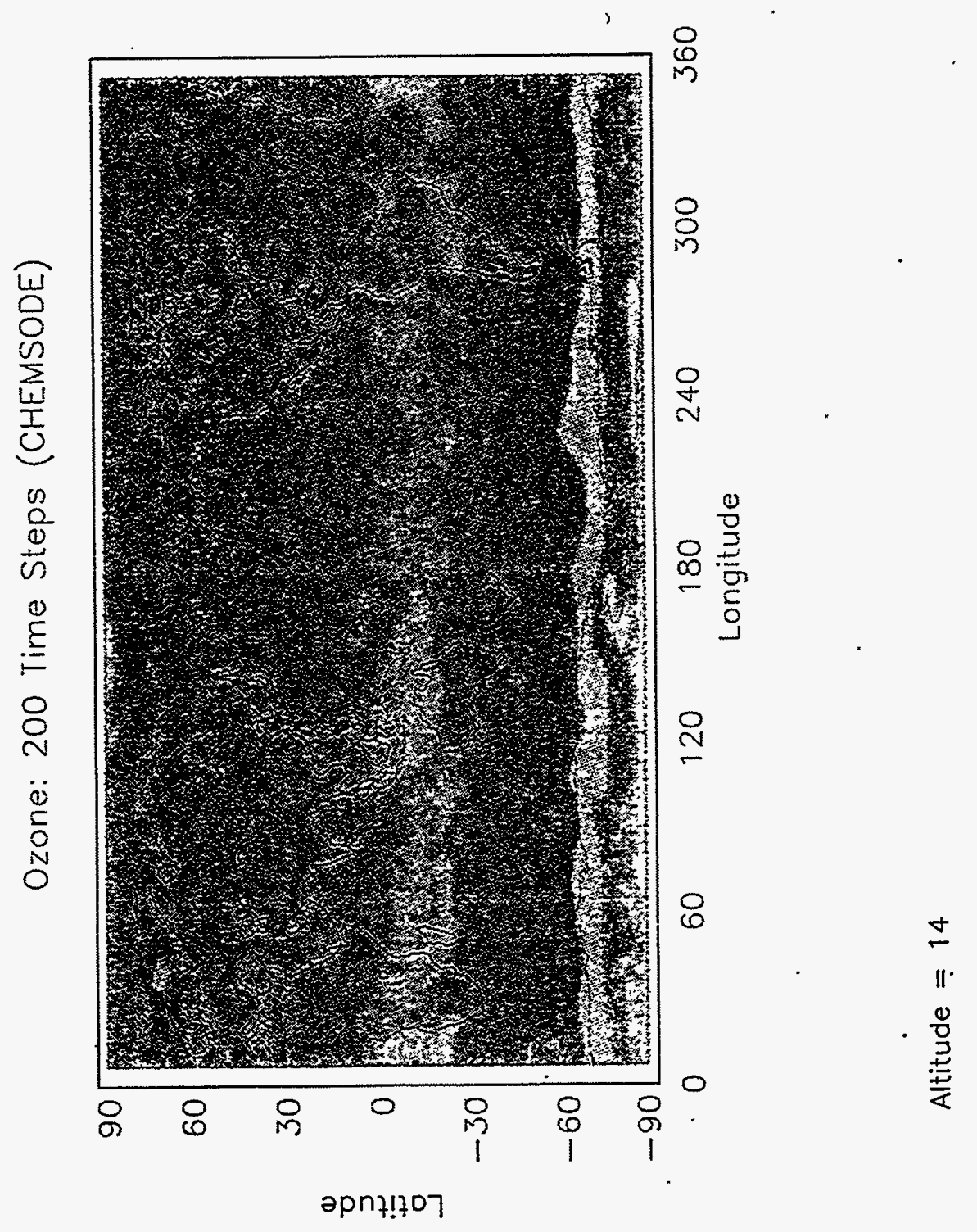


FIGURE 11. Ozone results for two hundred model steps at reference altitude 16.

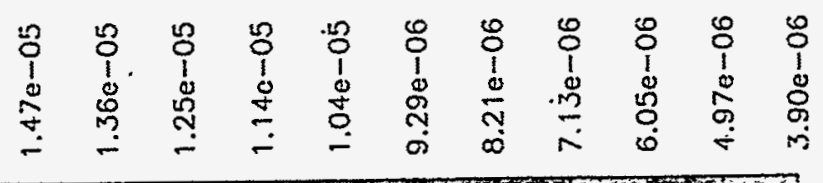

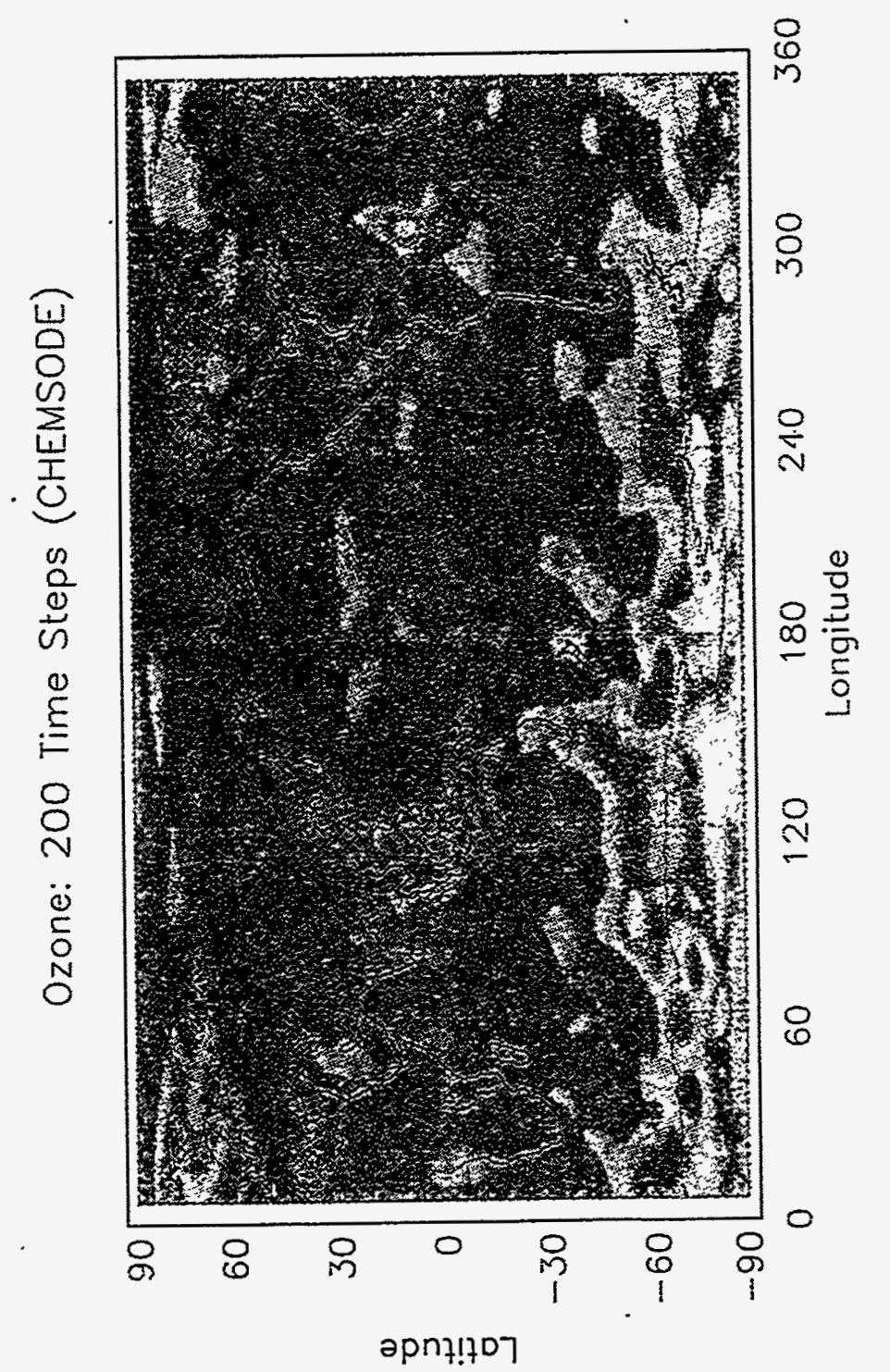

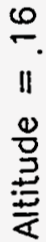




\subsection{2-D Model}

The LLNL 2-D CRT model has been described elsewhere ([43] for example). The chemical mechanism determines the distributions of 44 chemically active trace constituents in the troposphere and stratosphere [43, Appendix B]. This model provides a much larger chemical mechanism to test the preconditioned time difference techniques. For the runs presented here, the model has been modified to calculate fully diurnal photolysis rates at each timestep that the chemistry solver takes. Thus the model has been given a strong diurnal cycle for this study (In many of the previous studies $[36,43,72,75]$, it primarily used diurnally averaged rates).

The model was run for one full day of simulation, starting on the Winter solstice. Model timesteps were 15 minutes long. The same parameters used for the 3-D runs were used for the 2-D runs. Times on the CRAY C-90 are presented in Table IV.

TABLE IV. Timings for the diurnal version of the LLNL 2-D model.

\begin{tabular}{lll}
\hline CHEMSODE & LSODE & Speedup \\
2569 seconds & 15117 seconds & 5.88
\end{tabular}

Table IV shows CHEMSODE to be nearly six times as fast as LSODE for this model. This is likely due to both the larger chemical mechanism and the calculation of photolysis rates at each step.

\subsubsection{Results}

The figures presented in this section show the percent difference in CHEMSODE's and LSODE's computed values for the 2-D calculations. Figure 12 begins with the percent difference in ozone, and demonstrates that, às with the 3-D model, CHEMSODE's accuracy is well within the required one percent. The difference is zero for most of the atmosphere, with the exception of the extreme lower and upper altitudes shown in the graph. In those sections of the atmosphere, the discrepancy is still only a few hundredths of a percent.

Figure 13 shows the error plot for the $\mathrm{OH}$ radical. This species has a very strong diurnal cycle and although the error is nonzero everywhere, it is again well within the one percent 
error criterion. Figure 14 shows a similar plot for $\mathrm{NO}_{2}$, while Figure 15 shows the plot for CO. Again, the error is within acceptable tolerance in both cases. Plots for methane and NO proved to be very uninteresting since the error in these cases was zero everywhere.

In Figure 16, a plot of the error in the chlorine atom is shown. This species had one of the highest errors in the mechanism. The contours in the 60 degree latitude range at $20 \mathrm{~km}$ altitude show an error of about seven tenths of a percent. Even though this error is higher than most, it is again well within the acceptable limit of one percent. The bromine atom (Figure 17) also had a higher error than most, while $\mathrm{HCl}$ (Figure 18) was exceptionally accurate.

Finally, Figures 19 through 23 demonstrate the results of the run for $\mathrm{OH}$ at five different hours during the calculation. This ion has a very strong diurnal cycle, and is not present during nighttime hours. Figure 19 shows the initial condition (at 1 PM). Note the absence of $\mathrm{OH}$ in the North Polar region of the atmosphere, due to the 24 -hour night in that region. Figure 20 shows the state of the atmosphere at 7 PM. The sun has.set in the lower atmosphere and has set in a large portion of the upper atmosphere. A disappearance of $\mathrm{OH}$ in these areas is noted. In Figure 21 (3 AM), only the South Pole (which has 24 hour sunlight) has any concentration of $\mathrm{OH}$ left. Figure 22 shows the atmosphere at $7 \mathrm{AM}$ and the resulting reappearance of the $\mathrm{OH}$ ions. Figure 23 shows the calculation at noon the following day and is almost identical to the initial condition 


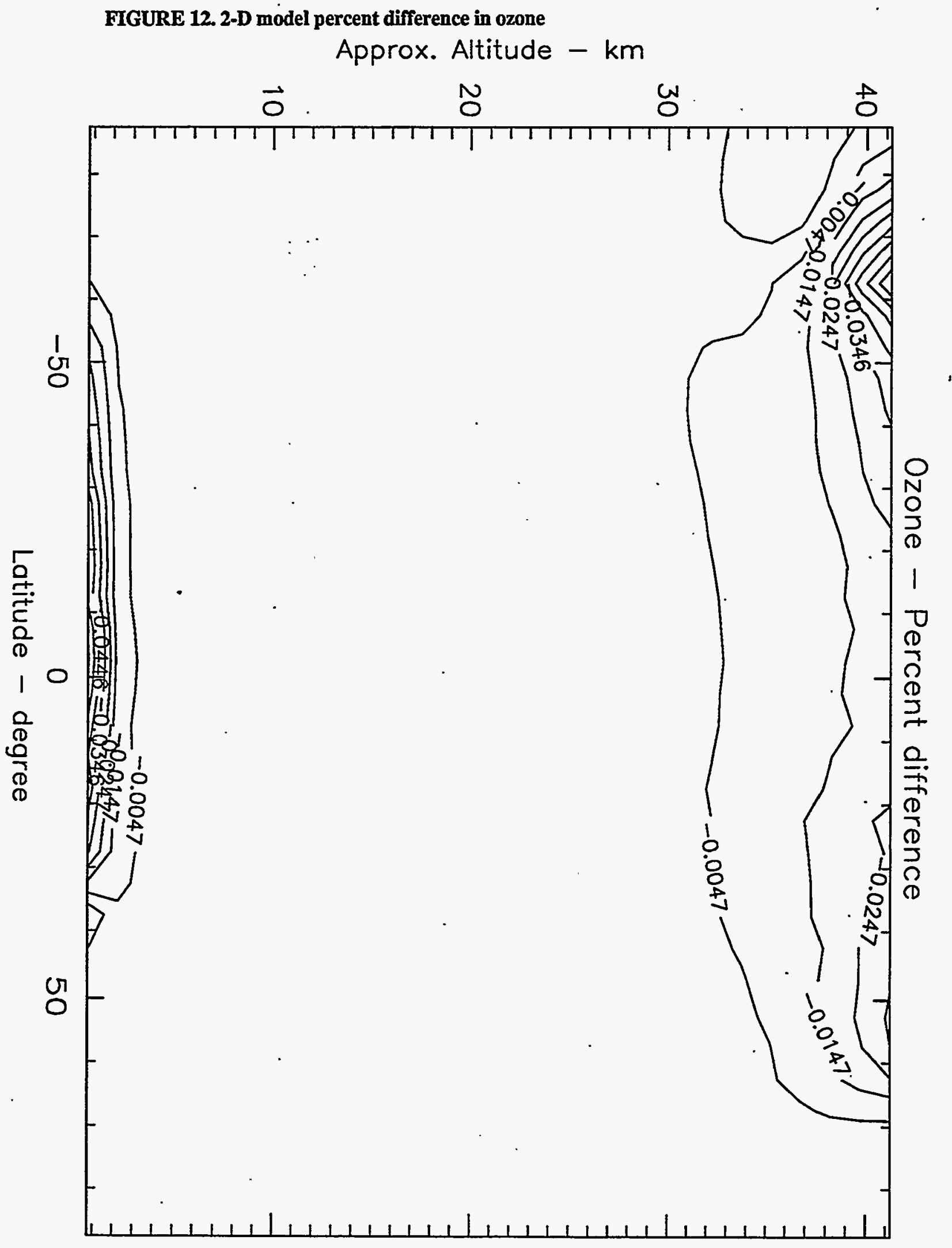


FIGURE 13. 2-D model percent difference in $\mathrm{OH}$

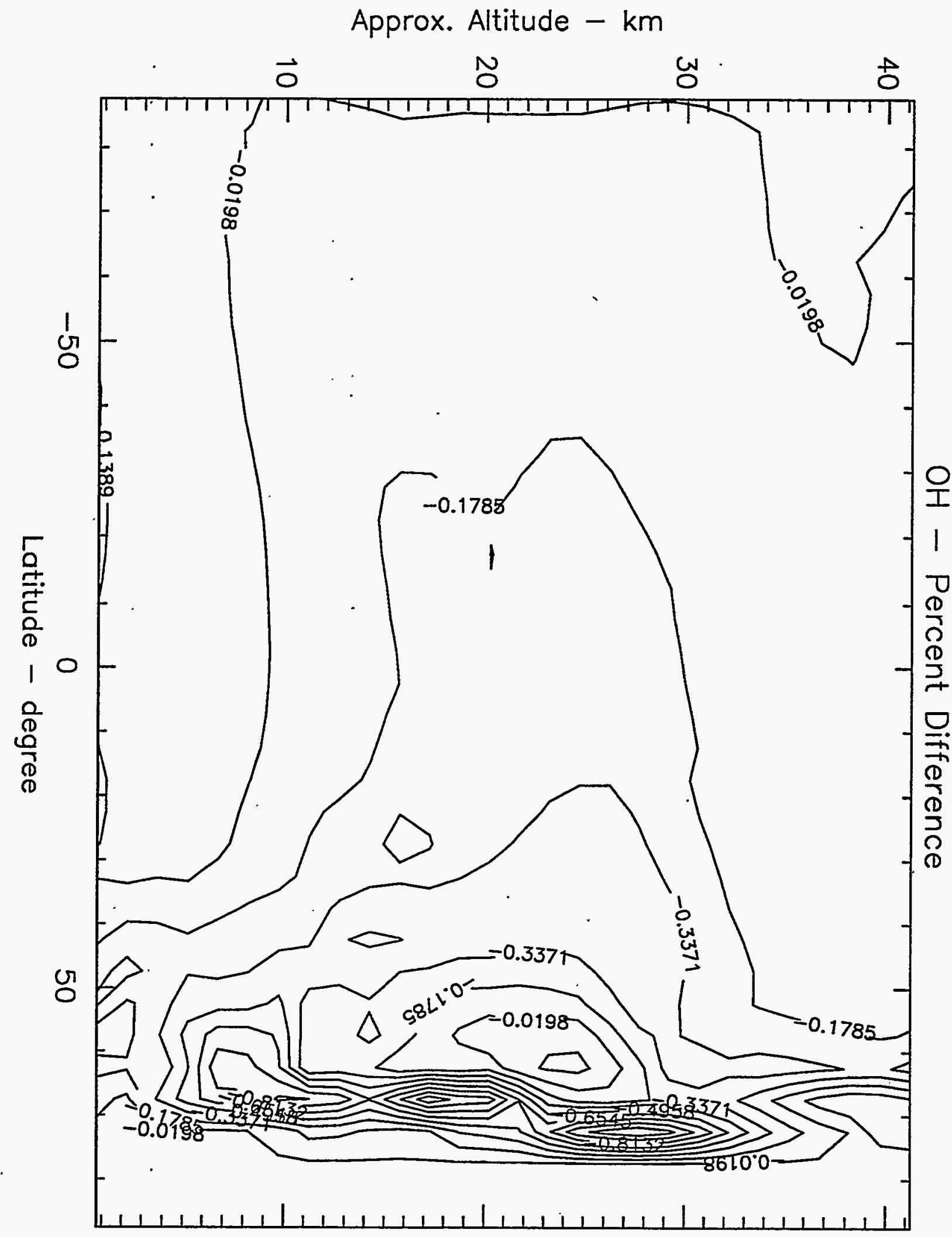




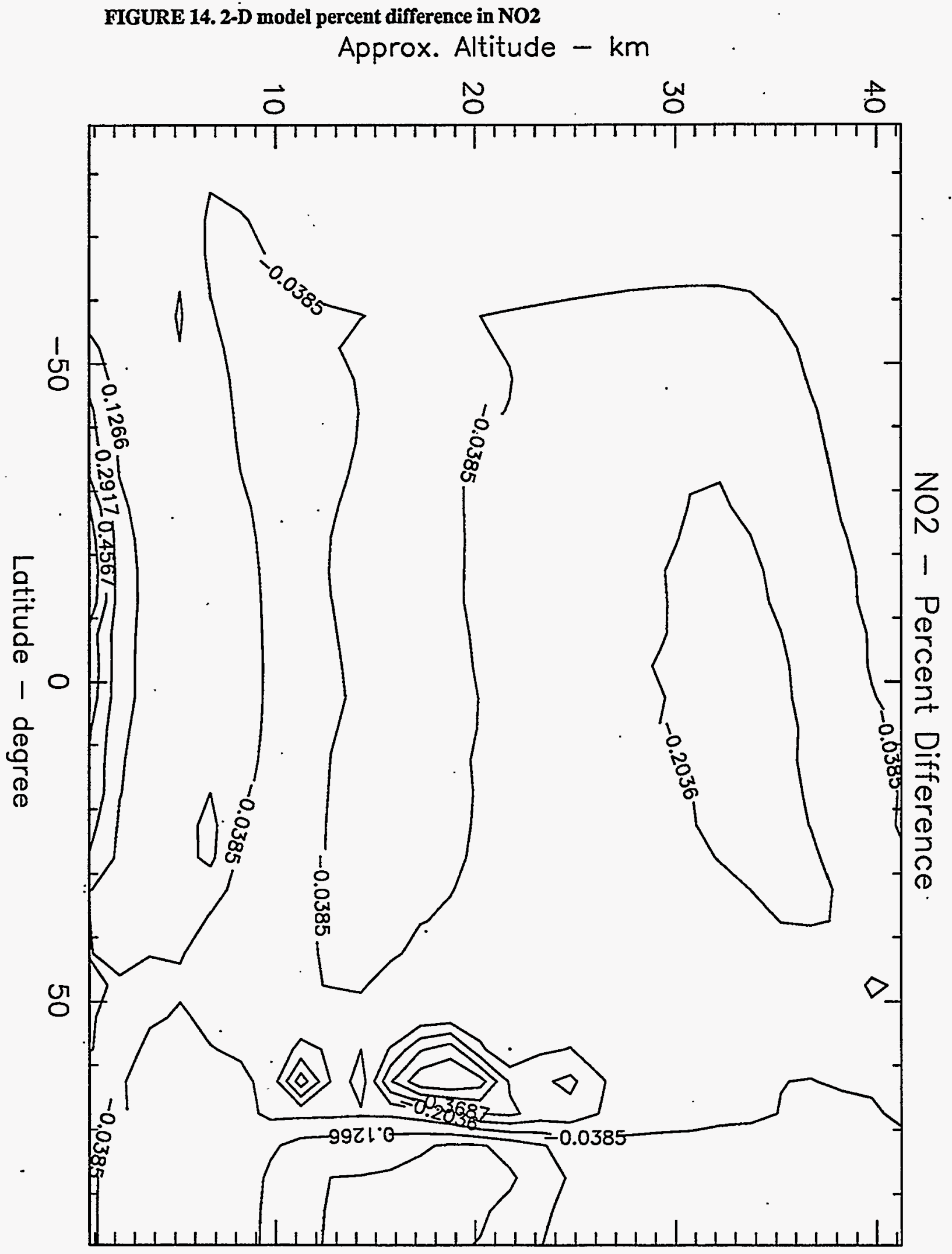




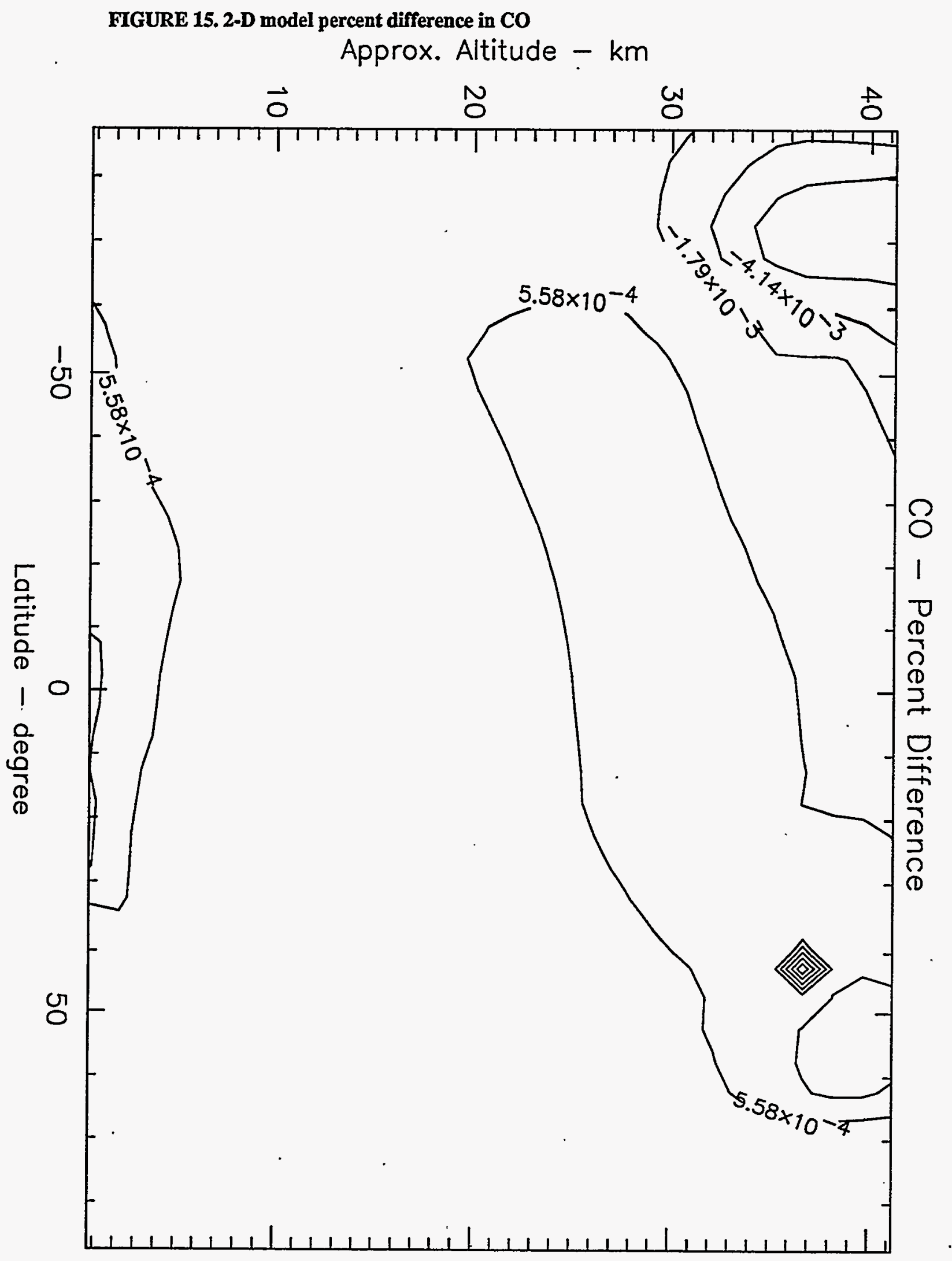


FIGURE 16. 2-D model percent difference in $\mathrm{Cl}$

Approx. Altitude - km

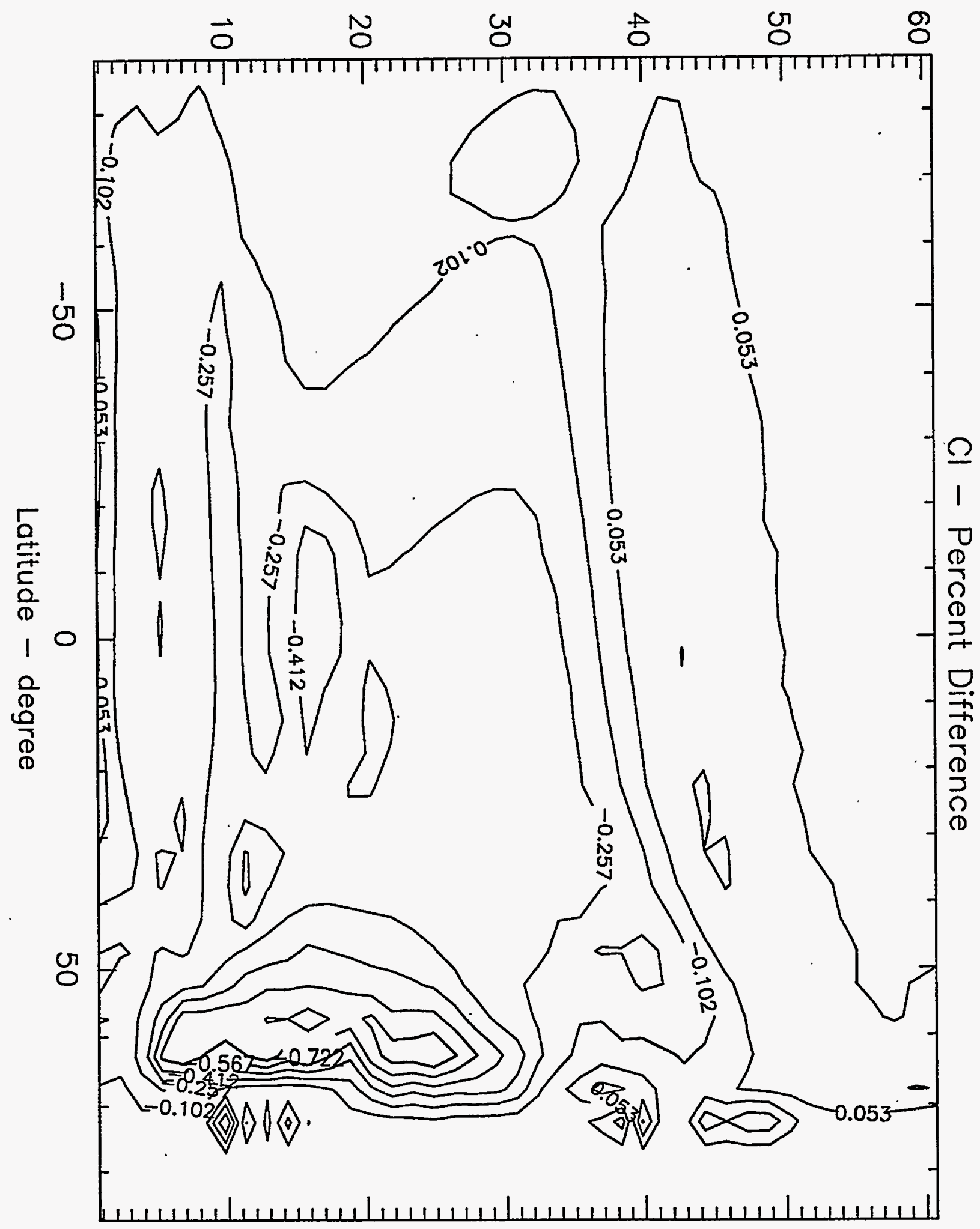


FIGURE 17. 2-D model percent difference in Bromine

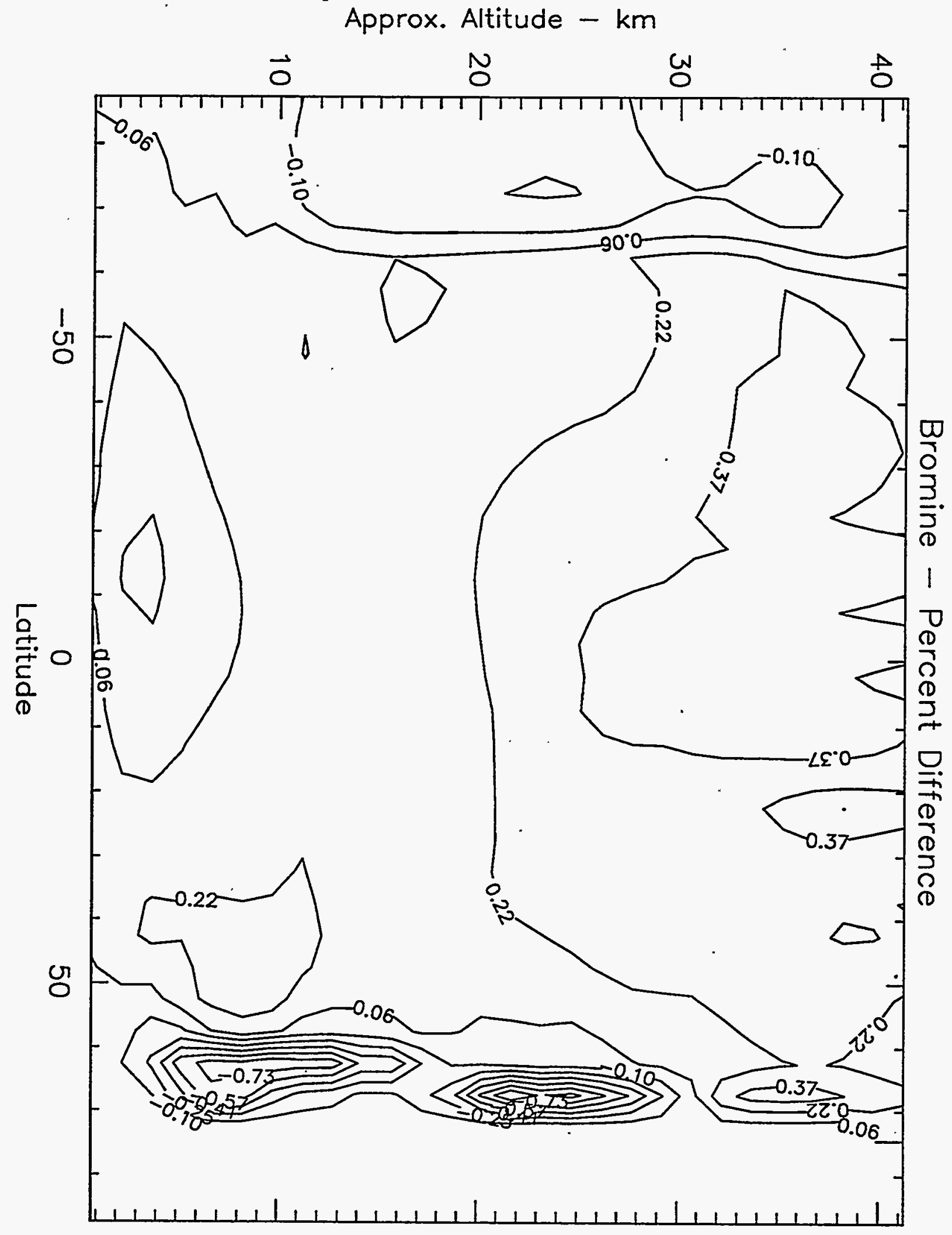


FIGURE 18. 2-D model percent difference in $\mathrm{HCl}$

$$
\text { Approx. Altitude - } \mathrm{km}
$$

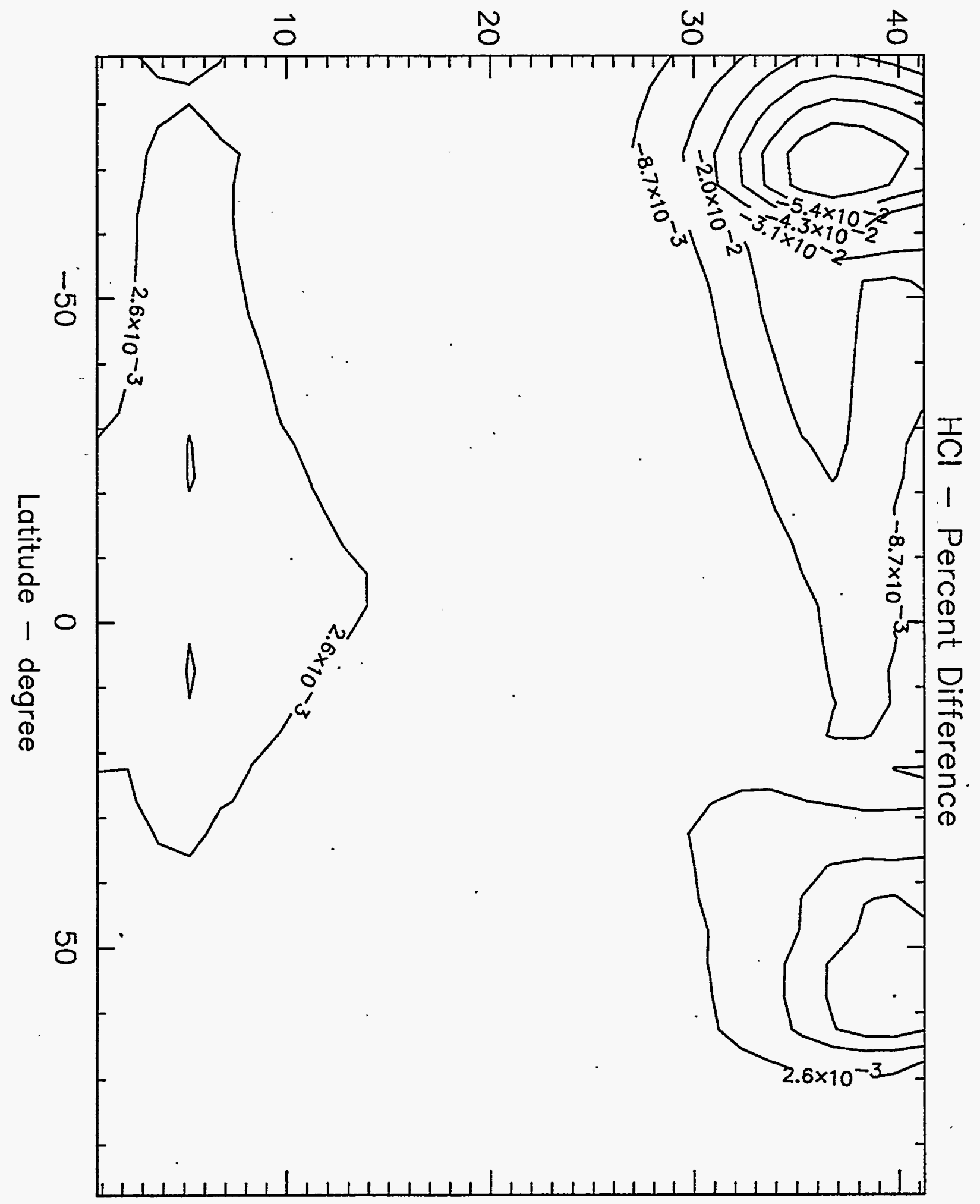


FIGURE 19. 2-D model OH at 1 PM

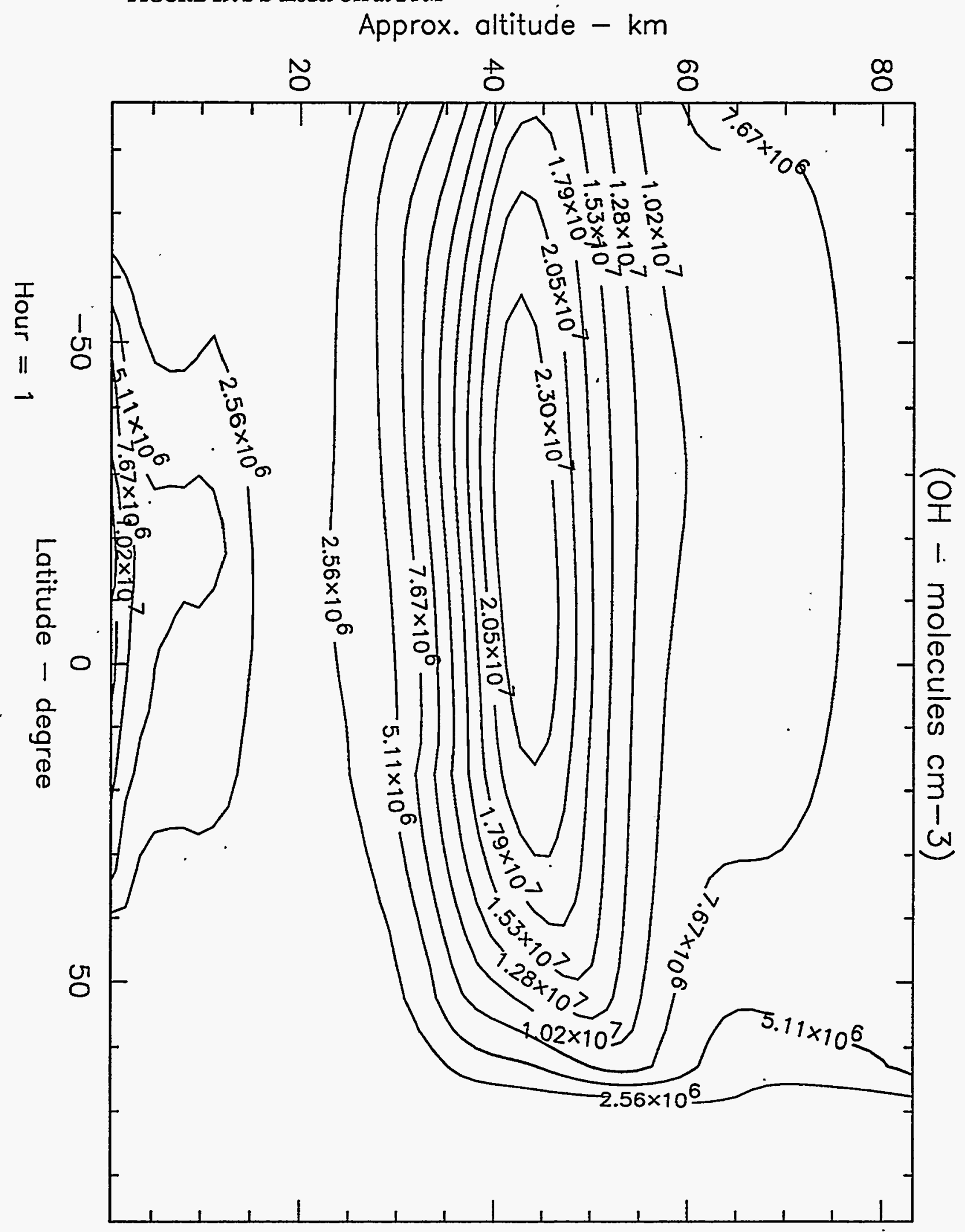


FIGURE 20. 2-D model OH at 7 PM

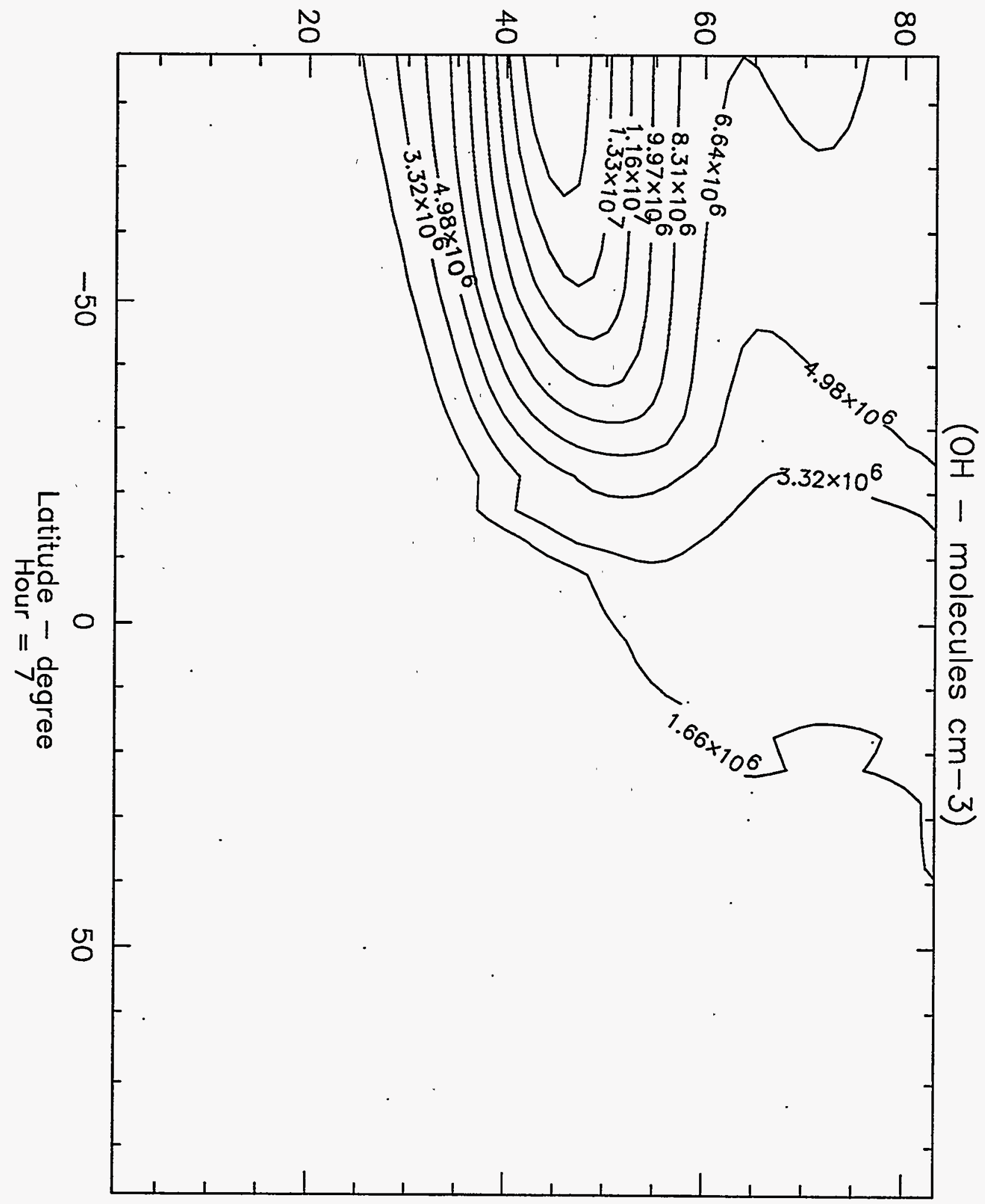




\section{FIGURE 21. 2-D model OH at $3 \mathrm{AM}$}

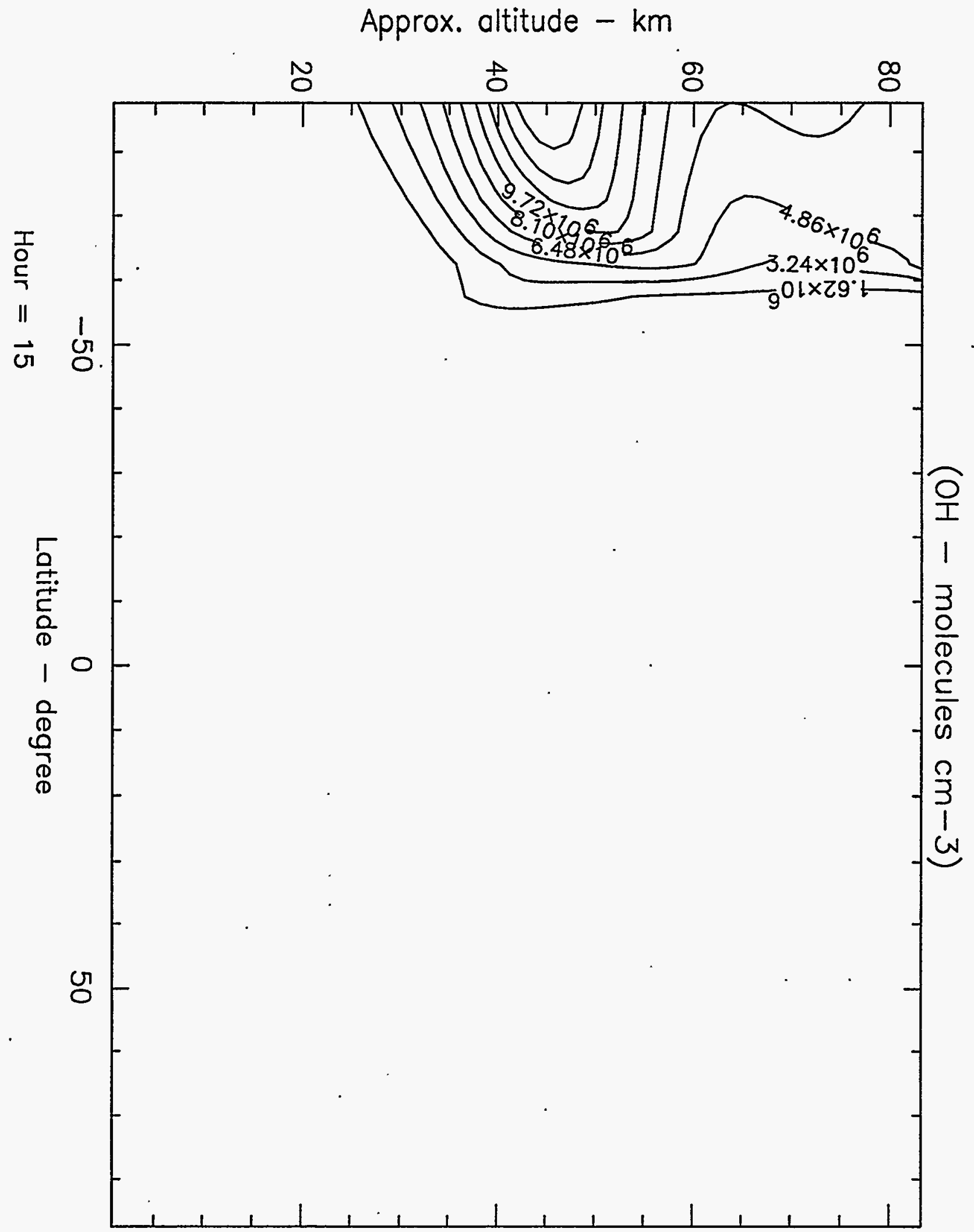


FIGURE 22. 2-D model OH at 7 AM

Approx. altitude - $\mathrm{km}$

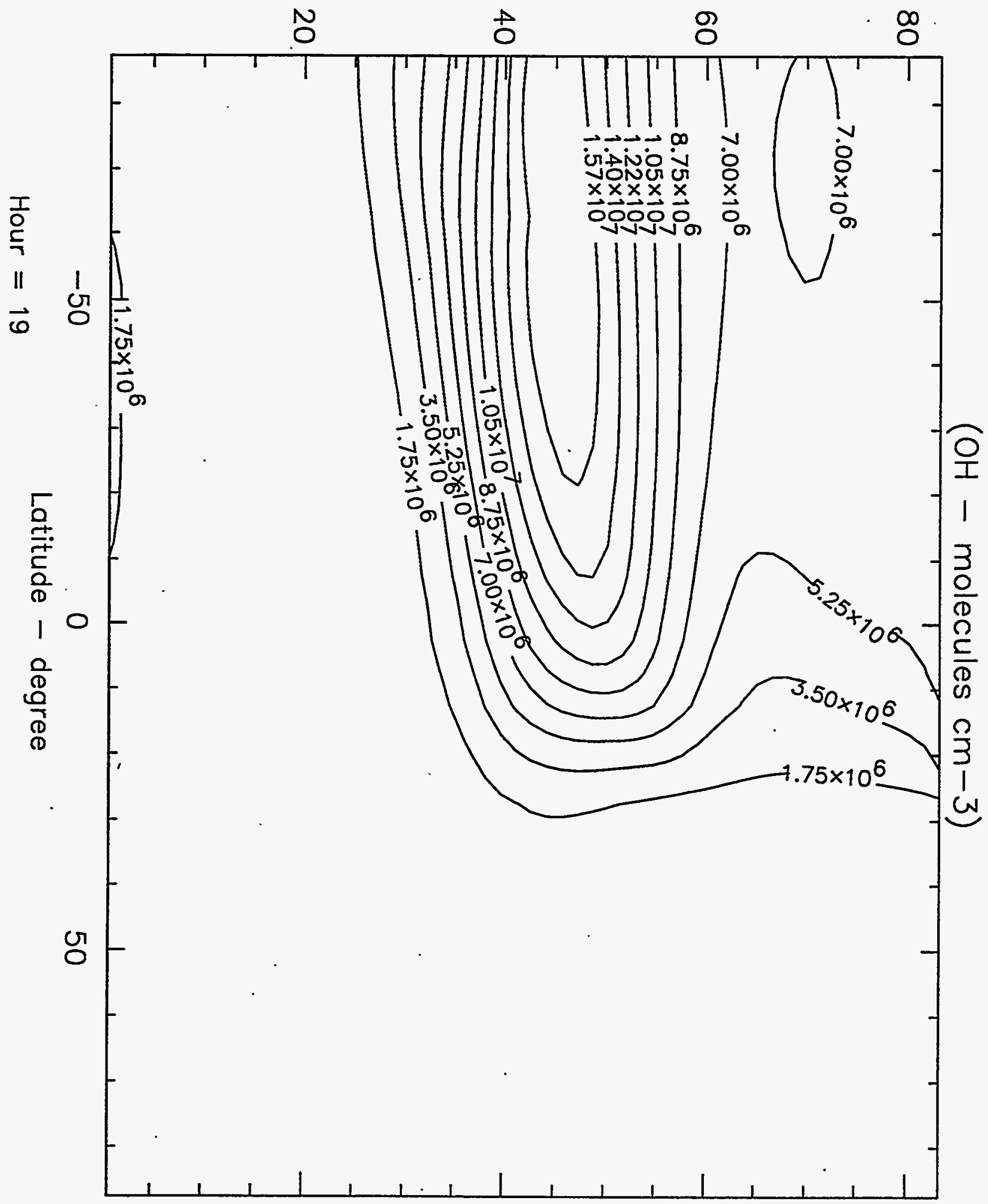


FIGURE 23. 2-D model OH at 12 PM

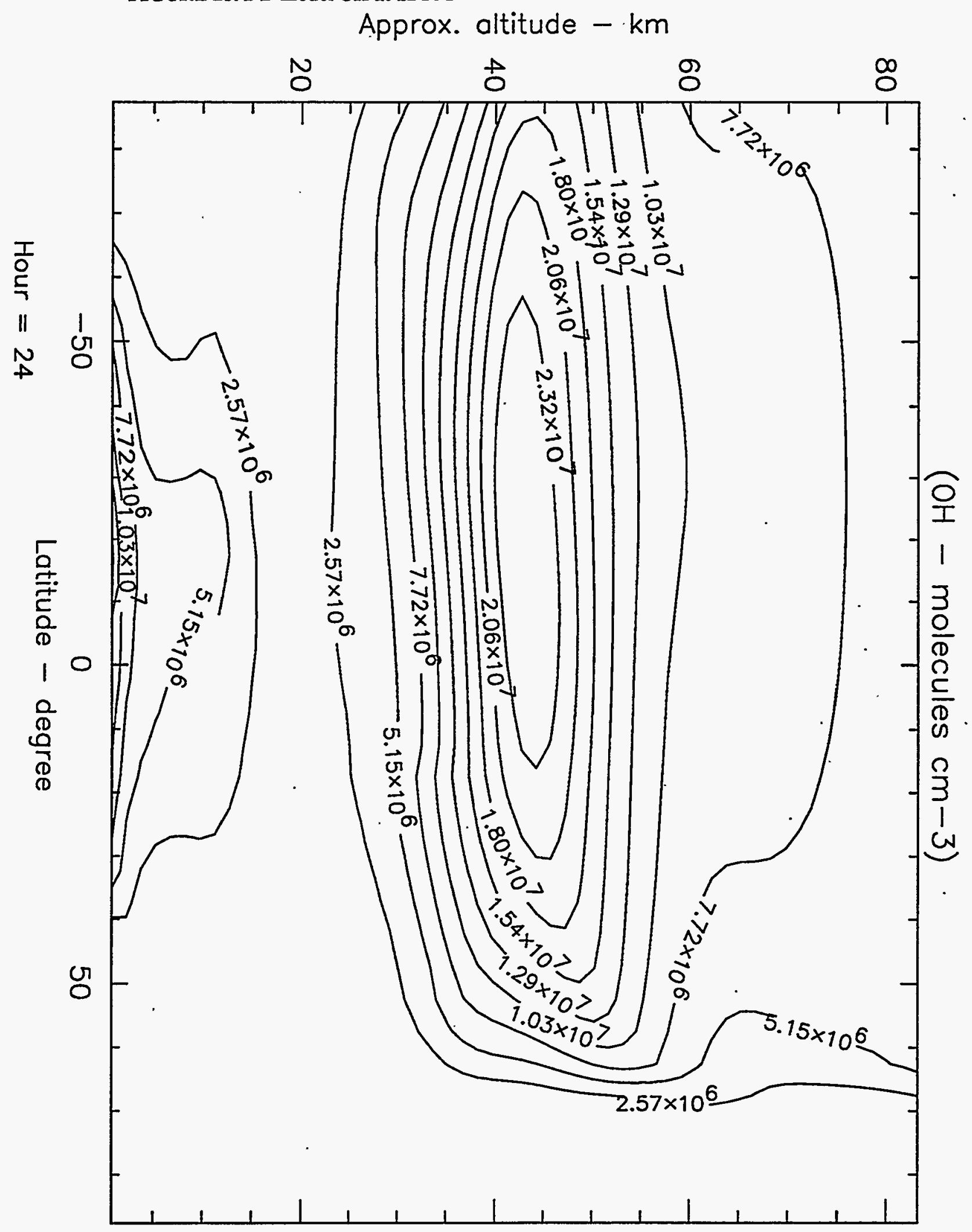




\subsection{Conclusions}

The number of chemically reacting. species in the 3-D model was very small, so at first glance, it would appear that CHEMSODE's advantage would be quite limited. CHEMSODE employs an explicit technique that eliminates the need for an implicit solve. Thus, the bigger the ODE system, the bigger CHEMSODE's advantage over the classic implicit methods employed by LSODE. Even running a modest eight species model, however, CHEMSODE proved to be significantly faster. In this chapter (unlike the previous chapter), LSODE was allowed to use up to its full fifth order accurate techniques, while CHEMSODE used its fixed second order technique. In using the 2-D model, CHEMSODE's superior speed was confirmed as was its accuracy. It is clear that CHEMSODE is superior to LSODE for use in diurnal CRT models. 
CHAPTER 4

Conclusions and Future Direction

\subsection{Summary: Results of this Study}

Atmospheric CRT models are vital to research on the global climate and atmospheric chemical change. At this point, fully diurnal 3-D CRT models are still somewhat prohibitive in the enormous amount of CPU time required to perform even the most modest runs. The majority of CPU effort in running these models is the solution of the chemical kinetic ODE system, which is nonlinear and very stiff.

Several approaches are available to reduce the stiffness of the ODE system, in particular the family approach or the assumption of steady state. Although numerically beneficial, these methods are approximations whose implications are not well understood and can be limiting as to the results obtainable to the models that employ them. Thus, the overwhelming majority of effort has concentrated on research into specialized numerical techniques for treating these stiff kinetics equations. This study attempted to proceed along these lines by not only producing a fast solution technique, but by doing so without the use of a Jacobian matrix. This makes the preconditioned time differencing techniques attractive from a storage standpoint as well.

Simple Jacobi and Gauss Seidel preconditioning of the backward Euler and trapezoid rule techniques resulted in explicitly computable, unconditionally stable methods for atmo- 
spheric chemical kinetics problems. This study showed Jacobi to be the clear choice for equations with strong diurnal cycles. It is clear that Jacobi is the preconditioner of choice in a global atmospheric chemical-radiative-transport model. This algorithm has led to the development of the CHEMSODE numerical ODE package for the robust solution of these chemical kinetics equations. CHEMSODE has been created for kinetics equations in the same spirit as the more general LSODE and VODE [10] packages (which apply to the general ODE problem). CHEMSODE's implementation of the Jacobi preconditioned trapezoid rule outperformed the ODEPACK routine LSODE by an average $20 \%$ in a realistic chemistry scenario taken from a full chemical-radiative-transport model, even with the large amount of overhead that was required to perform the radiative calculations. These results laid the foundation for the use of these methods with full CRT models with strong diurnal cycles.

The use of CHEMSODE with a 3-D CRT model was examined on vector and parallel architectures. CHEMSODE outperformed the LSODE package by a factor of two in several cases. This result is encouraging both because of the small number of species modeled in the 3-D code and because of the advantage in method order LSODE was allowed to enjoy over the CHEMSODE solver. CHEMSODE's advantage is that it eliminates the use of a Jacobian matrix and the need for an implicit solve. Thus, one would believe that the greater the number of species involved (i.e. the greater the size of the nonlinear system that the classic methods would have to solve), the greater CHEMSODE's speed advantage. Even with a small number of species, this study has shown that CHEMSODE enjoys a respectable advantage. Further, during these runs, LSODE was allowed to use up to fifth order methods, while CHEMSODE used a fixed second order method. Thus, the development of higher order preconditioned techniques should stretch the margin of superiority even further. Also shown was the high degree of parallelism achievable with these models. It is doubtful that we could design a better problem for demonstrating the advantages of parallel computing. 


\subsection{Future Direction}

The results of this study lay the foundation for further work in several directions. Most obvious is the development of higher order preconditioned techniques together with the robust software to implement them. LSODE and VODE vary the method that they use from first up to fifth order. As error control for the preconditioned techniques becomes better understood, such variable order algorithms can be developed for the preconditioned schemes to bring them toward the order of accuracy of the standard solvers used for comparison in this study. The issue of cheap, accurate error control is not an easy one however.

Another obvious direction for additional research is the development of higher performance preconditioners. This study examined the two most obvious and straightforward preconditioners available, namely Jacobi and Gauss Seidel. These two were attractive because they did not require any knowledge of the Jacobian matrix. There are, of course, other possibilities. An attempt to bridge the gap between the "full Jacobian" and "no Jacobian" approaches would be the obvious "partial Jacobian" approach (see, for example [59, 61]). The form of these kinetics equations makes the diagonal band of the Jacobian readily available analytically. This could certainly be employed in a decoupling of the equations (in the Jacobi sense) followed by a Newton linearization of the resulting series of scalar equations, for example. There is certainly no shortage of research to be done in this area.

There are also implementation issues to be improved. There exist implementations of the BDF formulas which take great advantage of vector architectures by vectorizing around grid cell dimension [33]. The same attention can be given to the preconditioned techniques. Given the same.efficient implementation, the performance of the grid-cell vectorized codes can be bettered in this way.

There is also the possibility of applying preconditioned techniques to the PDE conservation equations. This study relied on the operator splitting principle to separate out the equations of the chemical kinetics. This allowed the development of the specialized 
numerical techniques examined here. Recent efforts have seen some success in the development of hybrid techniques for the entire non-split PDE conservation equation system [65A]. This approach, if successful, is in many ways more desirable than the approach used in this study, since splitting is not completely understood at this time.

Finally, there are other application areas outside of the atmospheric sciences. Combustion is an excellent example [39], since many combustion codes are at least as complex as atmospheric CRT models. These combustion codes often have many more species than do the atmospheric codes, so much so that the memory required to run a solver like LSODE becomes prohibitive. CHEMSODE's lack of a Jacobian matrix makes this very nearly a non-issue for the preconditioned techniques.

In summary, as with any "young" technique, there is a large amount of knowledge yet to be gained with respect to the preconditioned time difference techniques. Issues ranging from the development of theory and more accurate, higher order methods, to error control and robust, efficient implementation, to the examination of their suitability in other application areas are yet to be fully exhausted. This study has shown, however, that these techniques promise to deliver significant improvement over the classic linear multistep methods currently in wide use with chemical kinetics codes. 
[1] G.D. Akrivis, V.A. Dougalis, and O.A. Karakashian, "On Fully Discrete Galerkin Methods of Second-Order Temporal Accuracy for the Nonlinear Schroedinger Equation", Numerische Mathematik, 59, 1991

[2] D.J. Allen, A.R. Douglass, R.B. Rood, and P.D. Guthrie, “Application of a Mono- tonic Upstream-biased Transport Scheme to Three-Dimensional Constituent Transport Calculations", Mon. Wea.Rev., 119, 1991

[3] C.J. Aro, "CHEMSODE: A Stiff ODE Solver for the Equations of Chemical Kinetics", Report UCRL-ID-119557, Lawrence Livermore National Lab, Livermore, CA, 1995

[4] C.J. Aro and G.H. Rodrigue, "Preconditioned Time Differencing for Stiff ODEs in Diurnal Atmospheric Kinetics", Report UCRL-JC-118092, Lawrence Livermore National Lab, Livermore, CA, 1994 (in Press, Comput. Phys. Commun.)

[5] J. Austin, "On the explicit versus family solution of the fully diurnal photochemical equations of the stratosphere", J. Geophys. Res., 96, 12, 1991

[6] J.P. Boris and E.S. Oran, Numerical Simulation of Reactive Flow, Elsevier, 1987 
[7] G. Brasseur and S. Solomon, Aeronomy of the Middle Atmosphere, D. Reidel, Dordrecht, 1984

[8] K.E. Brennan, S.L. Campbell, and L.R. Petzold, Numerical Solution of Initial Value Problems in Differential-Algebraic Equations. North Holland, Amsterdam, 1989

[9] P.N. Brown, G.D. Byrne, and A.C. Hindmarsh, "VODE, a Variable Coefficient ODE Solver”, SIAM J. Sci. Stat. Comput., 10, 1989

[10] P.N. Brown and A.C. Hindmarsh, "Reduced Storage Matrix Methods in Stiff ODE Systems", J. Appl. Math. Comput., 31, 1, 1989

[11] G.D. Byme and A.C. Hindmarsh, "A Polyalgorithm for the Numerical Solution of Ordinary Differential Equations", ACM Trans. Math. Software, 1, 1, 1975

[12] G. D. Byrne and A. C. Hindmarsh, "Stiff ODE Solvers: A Review of Current and Coming Attractions", J. Comput. Phys., 70, 1, 1987

[13] J.S. Chang, A.C. Hindmarsh, and N.K. Madsen, "Simulation of Chemical Kinetics Transport in the Stratosphere", Stiff Differential Systems, Plenum, NY, 1974

[14] T.J. Chung and G.R. Karr, editors, Finite Element Analysis in Fluids, (Proceedings of the Seventh International Conference on Finite Element Methods in Flow Problems), UAH Press, Huntsville, AL, 1989

[15] M.G. Crandall and A. Majda, "The Method of Fractional Steps for Conservation Laws", Math. Comp., 34, 1980

[10] P.J. Crutzen, "Ozone Production Rates in an oxygen-hydrogen-nitrogen oxide atmosphere”, J. Geophys. Res., 76, 1971

[17] C.F. Curtis and J.O. Hirschfelder, "Integration of Stiff Equations", Proc. Natl. Acad. Sci., USA, 38 (1952)

[18] D. Dabdub and J.H. Seinfeld, "Air Quality Modeling on Massively Parallel Computers", Atmos. Environ., 28, 1994 
[19] D. Dabdub and J.H. Seinfeld, "Extrapolation Techniques Used in the Solution of Stiff ODEs Associated with Chemical Kinetics of Air Quality Models", Atmos. Environ., 29, 3, 1995

[20] G. Dahlquist, "A Special Stability Problem for Linear Multistep Methods", BIT, 3, 1963

[21] J.E. Dennis Jr., and R. Schnabel, Numerical Methods for Unconstrained Optimization and Nonlinear Equations, Prentice Hall, Englewood Cliffs, NJ, 1983.

[22] R.P. Dickinson and R.J. Gelinas, "Sensitivity Analysis of Ordinary Differential Equation Systems-A Direct Method" J. Comput. Phys., 21, 123, 1976

[23] , S. Elliott, R.P. Turco, and M.Z. Jacobson, "Tests on combined projection/forward differencing integration for stiff photochemical family systems at long time step", Comput. Chem., 17, 1993

[24] C.W. Gear, "The Automatic Integration of Stiff Ordinary Equations", Proc. Int. Fed. Inform. Proc. Congr., Humanities Press, New York, NY, 1968

[25] C.W. Gear, Numerical Initial Value Problems in Ordinary Differential Equations, Prentice Hall, Englewood Cliffs, NJ, 1971

[26] G.H. Golub and C.F. Van Loan, Matrix Computations, The Johns Hopkins University Press, Baltimore, MD, 1983

[27] E. Hesstvedt, O. Hov, and I.S.A. Isaksen, "Quasi-steady state approximations in air pollution modeling: Comparison of two numerical schemes for oxidant prediction", Int.J. Chem. Kinet., 10, 1978

[28] A.C. Hindmarsh, "Linear Multistep Methods for Ordinary Differential Equations: Method Formulations, Stability, and the Methods of Nordsieck and Gear", Report UCRL-51186, Lawrence Livermore National Lab, Livermore, CA, 1972 
[29] A.C. Hindmarsh, "ODEPACK, A Systematized Collection of ODE Solvers", Report UCRL-88007, Lawrence Livermore National Lab, Livermore, CA, 1982

[30] J.R. Holton, "An Advective Model for Two-Dimensional Transport of Stratospheric Trace Species", J. Geophys. Res., 86, 1981

[31] B.G. Hunt, "Photochemistry of ozone in a moist atmosphere", J. Geophys. Res., 71, 1966

[32] D.J. Jacob, S. Sillman, J.A. Logan, and S.C. Wofsy, "Least independent variables method for simulation of tropospheric ozone", J.Geophys. Res., 94, 1989

[33] M.Z. Jacobson and R.P. Turco, "SMVGEAR: A Sparse-Matrix Vectorized GEAR Code for Atmospheric Models", Atmos. Environ., 28, 2, 1994

[34] J.H. Joseph, W.J. Wiscombe, and J.A. Weinman, "The delta eddington approximation for radiative flux transfer.", J. Atmos. Sci., 33, 1976

[35] R.J. Kee, L.R. Petzold, M.D. Smooke, and J.F. Grcar, Multiple Time Scales, J.V. Brackbill and B.I. Cohen editors, Academic, New York, 1985

[36] D.E. Kinnison, Effects of Trace Gases on Global Atmospheric Chemical and Physical Processes (Ph.D. Thesis), U.C. Berkeley, CA, 1989

[36A]A.A. Lacis, D.J. Wuebbles, and J.A. Logan, "Radiative Forcing of Climate by Changes in the Vertical Distribution of Ozone", J. Geophys. Res., 95, D7, 1990

[37] J.D. Lambert, Computational Methods in Ordinary Differential Equations, John Wiley and Sons, New York, NY, 1973

[38] G.J. McRae, W.R. Goodin, and J.H. Seinfeld, "Numerical solution of the atmospheric diffusion equation for chemically reacting flows", J. Comp. Phys., 45, 1982

[39] J.A. Miller, R.J. Kee, and C.K. Westbrook, "Chemical Kinetics and Combustion Modeling", Annu. Rev. Phys. Chem., 41, 1990 
[40] J.J. Monaghan, "Why Particle Methods Work", J. Sci. Stat. Comput., 3, 4, 1982

[41] J.J. Monaghan, “An Introduction to SPH”, Comput. Phys. Commun., 48, 1988

[42] R.J. LeVeque, Numerical Methods for Conservation Laws, Birkhaeuser, Berlin, 1992

[43] K.O. Patten, Jr., P.S. Connell, D.E. Kinnison, D.J. Wuebbles, T.G. Slanger, and L. Froidevaux, "Effects of Vibrationally Excited Oxygen on Ozone Production in the Stratosphere", J. Geophys. Res., 99, D1, 1994

[44] L.R. Petzold, "A description of DASSL: A differential/algebraic system solver", Report SAND82-8637, Sandia National Labs, Livermore, CA, 1982

[45] W.H. Press, S.A. Teukolsky, W.T. Vettering, and B.P. Flannery, Numerical Recipes in FORTRAN The Art of Scientific Computing (Second Edition), Cambridge University Press, NY, 1992.

[46] K. Radakrishnan and A.C. Hindmarsh, "Description and Use of LSODE, the Livermore Solver for Ordinary Differential Equations", Report UCRL-ID-113855, Lawrence Livermore National Lab, Livermore, CA, 1993

[47] G. Rodrigue and D. Wolitzer, "Preconditioned Time Differencing for the Parallel Solution of the Heat Equation", Proceedings of the Fourth SIAM Conference on Parallel Processing for Scientific Computing, SIAM Publishing, 1989

[48] R.B. Rood, "Numerical Advection Algorithms and Their Role in Atmospheric Transport and Chemistry Models", Rev. Geophys., 25, 1, 1987

[49] J.S. Rosenbaum, "Conservation properties of numerical integration methods for systems of ordinary differential equations", J. Comp.Phys., 20, 1976

[50] C. Sagan and J.B. Pollack, "An isotropic nonconservative scattering and the clouds of Venus", J. Geophys. Res., 72, 1967

[51] R.D. Saylor and R.I. Fernandes, "On the Parallelization of a Comprehensive Regional-Scale Air Quality Model", Atmos. Environ., 27A, 1993 
[52] L.F. Shampine, "Stiffness and the Automatic Selection of ODE Codes", J. Comput. Phys., 54, 1984

[53] L.F. Shampine and C.W. Gear, "A User's View of Solving Stiff Ordinary Differential Equations", SIAM Rev., 21, 1979

[54] T. Shimazaki and A.R. Laird, "A model calculation of the diurnal variation in minor neutral constituents in the mesosphere and lower thermosphere including transport effects", J. Geophys. Res., 75, 1970

[55] W.C. Shin and G.R. Carmichael, "Comprehensive Air Pollution Modeling on a Multiprocessor System", Comput. Chem. Engng., 16, 1992

[56] P.K. Smolarkiewicz, “A Fully Multidimensional Positive Definite Advection Transport Algorithm with Small Implicit Diffusion", J. Comput. Phys., 54, 1984

[57] J.I. Steinfeld, J.S. Francisco, and W.L. Hase, Chemical Kinetics and Dynamics, Prentice Hall, Englewood Cliffs, NJ, 1989

[58] J. Stoer and R. Burlisch, Introduction to Numerical Analysis, Springer, New York, 1980

[59] P.A. Stott and R.S. Harwood, "An implicit time-stepping scheme for chemical species in a global atmospheric circulation model", Ann. Geophysicae, 11, 1993

[60] G. Strang, "On the Construction and Comparison of Difference Schemes", J. Num. Anal., 5, 1968

[61] K. Suhre and R. Rosset, "Modification of a linearized semi-implicit scheme for chemical reactions using a steady-state-approximation”, Ann. Geophysicae, 12, 1994

[62] F. Thomasset, Implementation of Finite Element Methods for Navier-Stokes Equations, Sprịnger, New York, NY, 1981

[63] R.P. Turco and R.C. Whitten, "A comparison of several computational techniques for solving some common aeronomic problems", J. Geophys. Res., 79, 1974 
[64] B. van Leer, "Towards the ultimate conservative difference scheme. V: A second order sequel to Godanov's method.", J. Comput. Phys., 32, 1979

[65] J. G. Verwer. "Gauss-Seidel Iteration for Stiff ODEs from Chemical Kinetics", $J$. Sci. Comput., 15, 5, 1994

[65A]J.G. Verwer, J.G. Blom, and W. Hundsdorfer, "An Implicit-Explicit Approach for Atmospheric Transport-Chemistry Problems", Report NM-R9501, CWI, Amsterdam, 1995

[6] J.G. Verwer and M. van Loon, "An Evaluation of Explicit Pseudo-Steady State Approximation Schemes for Stiff ODE Systems from Chemical Kinetics", J. Comput. Phys., 113, 2, 1994

[67] J.G. Verwer and D. Simpson, "Explicit Methods for Stiff ODEs from Atmospheric Chemistry", Report NM-R9409, CWI, Amsterdam, 1994

[68] W.C. Wang, D.J. Wuebbles, W.M. Washington, R.G. Isaacs, and G. Molnar, "Trace Gases and Other Potential Perturbations to Global Climate", Rev. Geophys., 24, 1986

[69] R. Watson, and Ozone Trends Panel, M.J. Prather, and Ad Hoc Theory Panel, and M.J. Kurylo, and NASA Panel for Data Evaluation, "Present State of Knowledge of the Upper Atmosphere 1988: An Assessment Report", NASA Reference Publication 1208, 1988

[70] R.P. Wayne, Chemistry of Atmospheres (Second Edition), Clarendon Press, Oxford, 1991

[71] O.B. Widlund, "A Note on Unconditionally Stable Linear Multistep Methods", BIT, 7,1963 
[72] D.J. Wuebbles, P.S. Connell, K.E. Grant, R. Tarp, and K.E. Taylor, "Initial Results with the LLNL Two-dimensional Chemical-Radiative-Transport Model of the Troposphere and Stratosphere”, Report UCID-21178, Lawrence Livermore National Lab, Livermore, CA, 1987

[73] D.J. Wuebbles and J. Edmonds, Primer on Greenhouse Gases, Lewis, Chelsea, Michigan, 1991

[74] D.J. Wuebbles, K.E. Grant, P.S. Connell, and J.E. Penner, "The Role of Atmospheric Chemistry in Climate Change", APCA Journal, 39, 1, 1989

[75] D.J. Wuebbles, J.S. Tamaresis, and K.O. Patten, "Quantified Estimates of Total GWPs for Greenhouse Gases Taking Into Account Tropospheric Chemistry", Report UCRL-ID-115850, Lawrence Livermore National Lab, Livermore, CA, 1993

[76] T.R. Young and J.P. Boris, "A numerical technique for solving stiff ordinary differential equations associated with the chemical kinetics of reactive flow problems", $J$. Phys. Chem., 81, 1977 


\subsection{Code}

At this time, CHEMSODE uses a second order method (the trapezoidal rule) with a Jacobi preconditioner (one iteration). The package consists of the following subroutines:

\subsubsection{SOLVER}

SOLVER is the driver for the package. It takes the following arguments:

- F: (EXT) Name of the subroutine for the right hand side of the ODE system (the derivative). This is supplied by the user and must be declared as EXTERNAL in the calling program, having the form

SUBROUTINE F(NEQ, T, Y, P, IHAT, LBAR)

INTEGER NEQ

REAL T, $Y(N E Q)$, $P(N E Q)$, LHAT (NEQ) , LBAR (NEQ)

The inputs are NEQ, $T$ and $Y$ (the number of ODEs, current time and chemical species concentrations). $F$ is to set the chemical production and loss terms P(i), LHAT(i), LBAR(i).

- NEQ: (IN) The number of first order ODEs.

- Y: (INOUT) Array of components of the $Y(t)$ (dependent variable). $Y$ shall be dimensioned at least NEQ elements long. On initial call, it contains the species concentrations at $\mathrm{t}=\mathrm{T}$. On return, it contains the values at $\mathrm{t}=$ TOUT (see below). 
- $\mathrm{T}$ : (INOUT) Value of the independent variable. Upon return, it will contain the value TOUT.

- TOUT: (IN) The next point where output is desired.

- ATOL: (IN) Array of absolute error tolerances (size NEQ).

- RTOL: (IN) Array of relative error tolerances (size NEQ).

- RWORK: (WORK) This work array must be dimensioned at least $10 *$ NEQ real elements long. It is also used to pass optional arguments to the solver (see below IOPT).

- IOPT: (IN) Indicates whether the user has supplied optional input. IOPT $=0$ indicates no optional input. IOPT = 1 indicates that RWORK(1) contains the first stepsize to be attempted, RWORK(2) contains the maximum allowable stepsize, and RWORK(3) contains the minimum allowable stepsize. Default values recently used are RUMACH (see below), 1 second, and 1 hour respectively. A non-zero minimum is required since our "loose" method of step control has problems otherwise.

- ITASK: (IN) Indicates whether the call is a continue or a restart. ITASK $=0$ indicates a restart. ITASK $=1$ indicates a continue.

A flowchart for SOLVER is provided in Figure 2, and is very run-of-the-mill for this type of code. At this stage there is very little error checking and recovery, however the code has proven to be quite bullet-proof in our experiences. In adjusting the new stepsize, if two successive steps are rejected, the stepsize is automatically reduced to the minimum allowable; that is, the method is restarted.

\subsubsection{STEP2}

STEP2 takes a single discrete time step and returns an estimate of the error to the SOLVER routine. The stepsize attempted is specified by the SOLVER routine. The error estimate is based on the formula of Eq (18) in Chapter 2, section 2.2. This information is passed back to the SOLVER routine which calculates the weighted norm of the error and accepts or rejects the step accordingly (while adjusting the stepsize for the next try). 


\subsubsection{ADVANCE2}

ADVANCE2 implements the component equations for the preconditioned trapezoidal rule assuming that the production and loss rates are set. It essentially provides for the solution for $y_{i}^{n+1}$ assuming all other values are explicitly known (which they are). It uses a scalar newton iteration and a fixed number of iterations (two). This works well with a close enough initial guess ( $y_{i}^{n}-$ not to be confused with the initial guess for the preconditioner). Attempts at using the quadratic formula proved unsuccessful since it requires the subtraction of two positive quantities that are nearly equal.

\subsubsection{ADVANCE1}

ADVANCE1 implements the component equations for the preconditioned backward Euler method, again assuming that the production and loss rates are set. This is done in a manner identical with that in ADVANCE2.

\subsubsection{RUMACH}

RUMACH calculates the machine's unit roundoff in a machine independent manner.

FIGURE 1. CHEMSODE subroutine calling hierarchy.

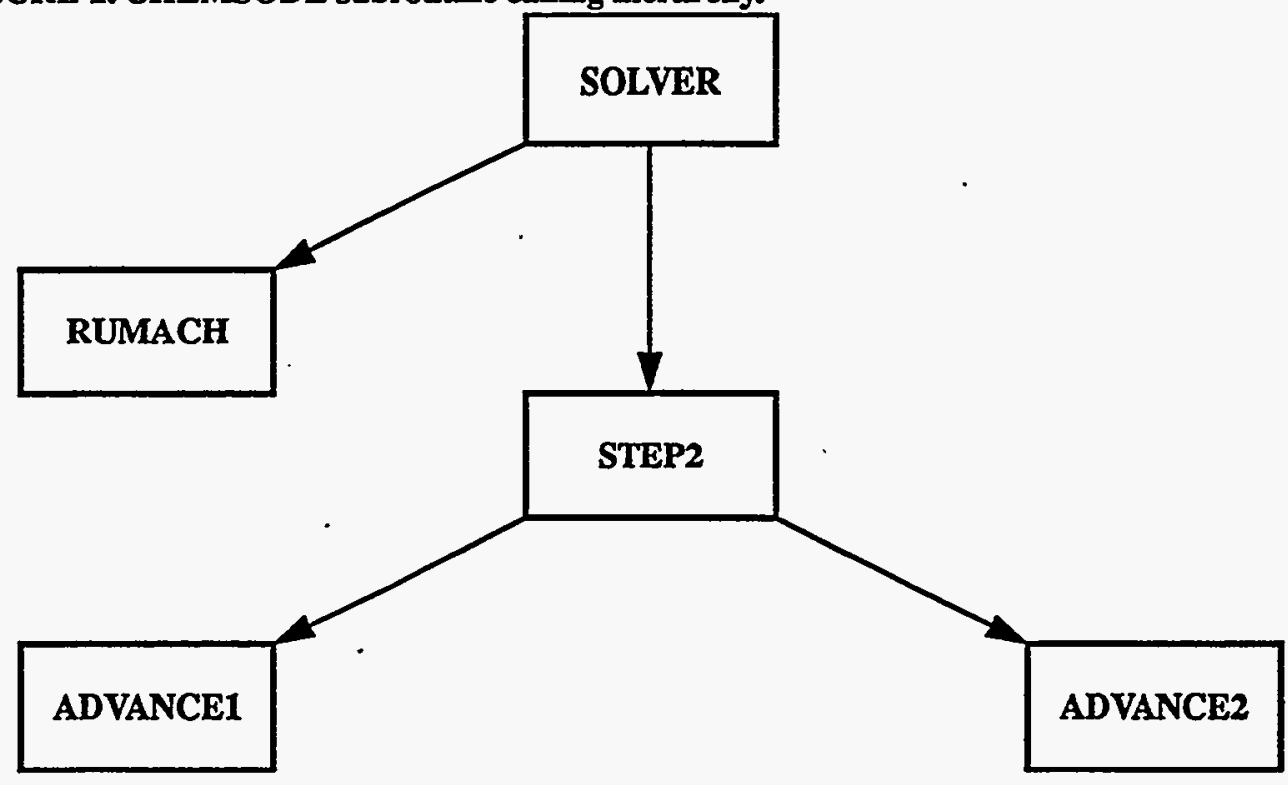


FIGURE 2. Flowchart for the CHEMSODE algorithm. (Subroutine SOLVER)

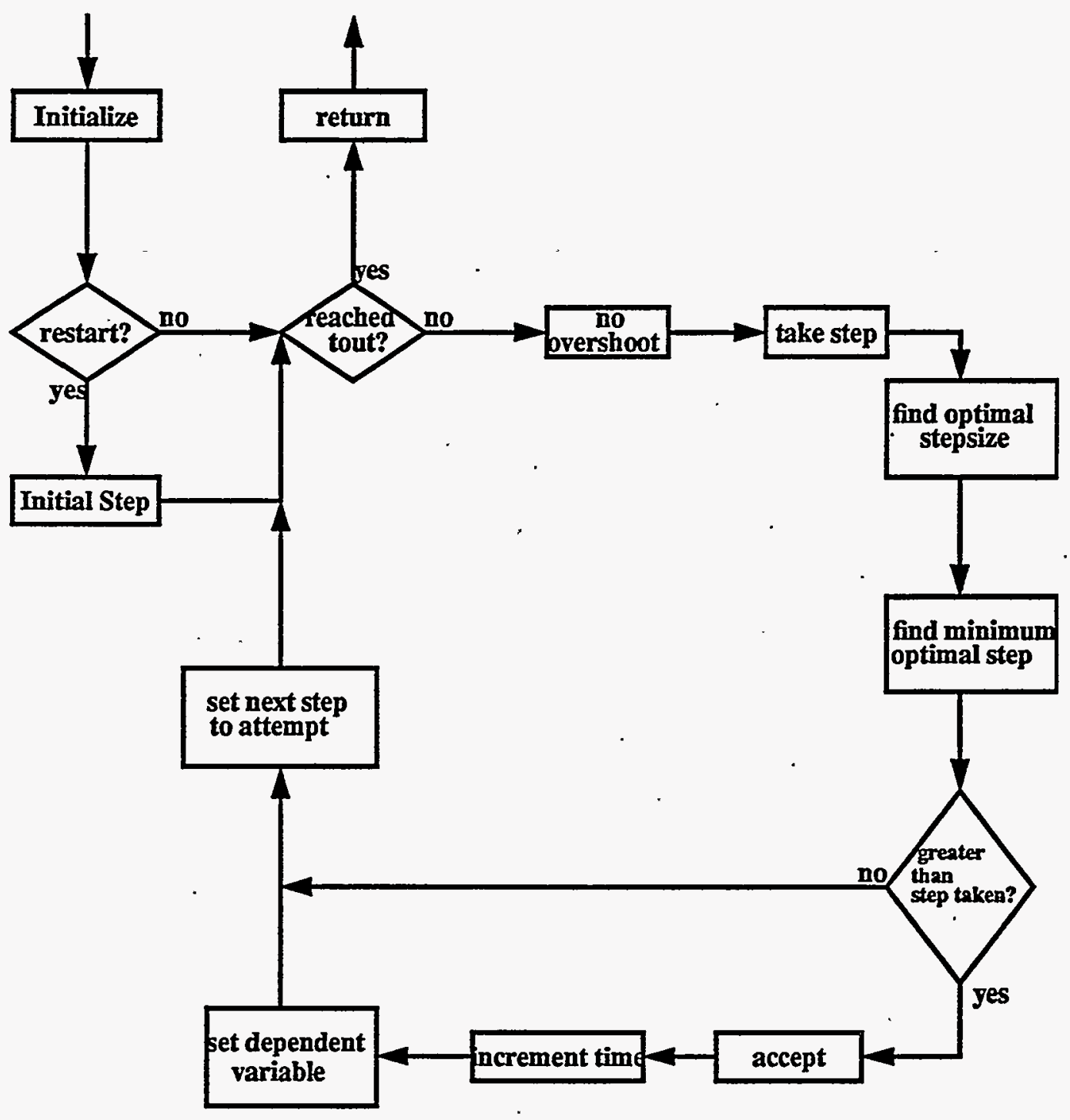




\subsection{Code usage and Example Problem}

The reader will find strong similarities between LSODE's calling sequence (and parameters) and CHEMSODE's. CHEMSODE's parameters shall be declared as follows:

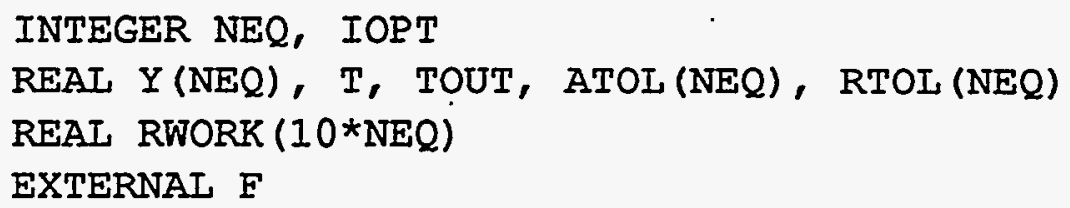

The solver is then called in the main program

CALI SOLVER (NEQ, F, Y, T, TOUT, ATOI, RTOL, 1 RWORK, IOPT, ITASK)

\subsection{EXAMPLE}

We use a simple problem from chemical kinetics. The Chapman atmosphere has been dealt with previously. It can be written in a simplified form (recall chapter 2) Eq. (42) for $y=\left[\begin{array}{l}y_{1} \\ y_{2}\end{array}\right]$, with

$$
\begin{gathered}
P(y, t)=\left[\begin{array}{c}
2 k_{3}(t) y_{3}+k_{4}(t) y_{2} \\
k_{1} y_{1} y_{3}
\end{array}\right] \\
\hat{L}(y, t)=\left[\begin{array}{cc}
k_{1} y_{3}+k_{2} y_{2} & 0 \\
0 & k_{2} y_{1}+k_{4}(t)
\end{array}\right] \\
(\bar{L}=0)
\end{gathered}
$$

and the constant values

$$
\begin{aligned}
& y_{3}=3.7 \times 10^{16} \\
& k_{1}=1.63 \times 10^{-16} \\
& k_{2}=4.66 \times 10^{-16}
\end{aligned}
$$


and

$$
k_{i}=\left\{\begin{array}{c}
\exp \left[\frac{-a_{i}}{\sin \omega t}\right], \sin \omega t>0, i=3,4 \\
0, \sin \omega t \leq 0
\end{array}\right.
$$

with

$$
a_{3}=22.62, a_{4}=7.601, \omega=\frac{\pi}{43200}
$$

This problem can be coded:

$$
\begin{aligned}
& \text { PROGRAM TEST } \\
& \text { INTEGER NEQ, IOPT } \\
& \text { REAI } Y(2), T \text {, TOUT, ATOL (2), RTOL (2) } \\
& \text { REAI RWORK (20) } \\
& \text { EXTERNAL FEX } \\
& I O P T=0 \\
& \text { ITASK }=0 \\
& \mathrm{NEQ}=2 \\
& \mathrm{~T}=0.0 \\
& \text { TOUT }=1.0 * 86400.0 \\
& \operatorname{ATOL}(1)=1.0 \mathrm{E}-2 \\
& \text { ATOL }(2)=1.0 \mathrm{E}-2 \\
& \text { RTOL (I) }=1.0000 \mathrm{E}-4 \\
& \text { RTOI }(2)=1.0000 \mathrm{E}-4 \\
& Y(1)=1.0 \mathrm{E} 6 \\
& Y(2)=1.0 \mathrm{E} 12 \\
& \text { PRINT *, 'VALUES AT TIME ', T, 'ARE ', Y } \\
& \text { END }
\end{aligned}
$$


SUBROUTINE FEX (NEQ, T, Y, P, LHAT, LBAR)

INTEGER NEQ

REAI T, $Y(*), P(*), \operatorname{LHAT}(*), \operatorname{LBAR}(*)$

REAL K1, K2, Y3

REAI K3, K4

REAL $K$

PARAMETER $(K 1=1.63 \mathrm{E}-16)$

PARAMETER (K2 $=4.66 \mathrm{E}-16)$

PARAMETER ( $(Y 3=3.7 \mathrm{E} 16)$

$P(1)=2.0 * K 3(T) * Y 3+K 4(T) * Y(2)$

$P(2)=K 1 * Y(1) * Y 3$

$\operatorname{LHAT}(1)=\mathrm{K} 1 * \mathrm{Y} 3+\mathrm{K} 2 * \mathrm{Y}(2)$

$\operatorname{LHAT}(2)=K 2 * Y(1)+K 4(T)$

$\operatorname{LBAR}(1)=0.0$

$\operatorname{LBAR}(2)=0.0$

RETURN

END

REAI FUNCTION K3 (T)

REAI T

REAI PI, OMEGA, A3

PARAMETER (PI = 3.14159265358979323846264338 )

PARAMETER (OMEGA $=\mathrm{PI} / 43200.0)$

PARAMETER $(A 3=22.62)$

REAL TEMP

$\operatorname{TEMP}=\operatorname{SIN}(T *$ OMEGA $)$

IF (TEMP .GT. 0.0$)$ THEN

$\mathrm{K} 3=\operatorname{EXP}(-\mathrm{A} 3 / \mathrm{TEMP})$

ELSE

$\mathrm{K} 3=0.0$

END IF 
RETURN

END

REAI FUNCTION K4 (T)

REAI T

REAI PI, OMEGA, A4

PARAMETER ( $P I=3.14159265358979323846264338)$

PARAMETER (OMEGA $=\mathrm{PI} / 43200.0$ )

PARAMETER $(A 4=7.601)$

REAI TEMP

TEMP $=\operatorname{SIN}(T *$ OMEGA $)$

IF (TEMP .GT. 0.0) THEN

$\mathrm{K} 4=\operatorname{EXP}(-\mathrm{A} 4 / \mathrm{TEMP})$

ELSE

$\mathrm{K} 4=0.0$

END IE

RETURN

END 


\subsection{The Box Model}

TABLE I. Chemically reactive species used in the box model.

\begin{tabular}{llllll}
\hline $\mathrm{O}_{3}$ & $\mathrm{~N}_{2} \mathrm{O}$ & $\mathrm{NO}$ & $\mathrm{NO}_{2}$ & $\mathrm{NO}_{3}$ & $\mathrm{~N}_{2} \mathrm{O}_{5}$ \\
$\mathrm{HONO}$ & $\mathrm{HNO}_{3}$ & $\mathrm{HO}_{2} \mathrm{NO}_{2}$ & $\mathrm{H}_{2} \mathrm{O}$ & $\mathrm{OH}$ & $\mathrm{HO}_{2}$ \\
$\mathrm{H}_{2} \mathrm{O}_{2}$ & $\mathrm{H}_{2}$ & $\mathrm{CH}_{4}$ & $\mathrm{CH}_{3} \mathrm{O}_{2}$ & $\mathrm{CH}_{3} \mathrm{OOH}$ & $\mathrm{CH}_{2} \mathrm{O}$ \\
$\mathrm{CO}$ & $\mathrm{CH}_{3} \mathrm{O}_{2} \mathrm{NO}_{2}$ & & & &
\end{tabular}

TABLE II. Chemical species assumed to be in steady state for the box model. $O\left({ }^{1} D\right) \quad$.

TABLE III. Thermal reactions in the Box Model

\begin{tabular}{lll}
\hline$O+O_{2}$ & $\rightarrow O_{3}$ \\
$O+O_{3}$ & $\rightarrow 2 O_{2}$ \\
$O\left({ }^{1} D\right)+N_{2}$ & $\rightarrow O+N_{2}$ \\
$O\left({ }^{1} D\right)+O_{2}$ & $\rightarrow O+O_{2}$ \\
$O\left({ }^{1} D\right)+O_{3}$ & $\rightarrow 2 O_{2}$ \\
$O\left({ }^{1} D\right)+O_{3}$ & $\rightarrow O_{2}+2 O$
\end{tabular}


TABLE III. Thermal reactions in the Box Model

\begin{tabular}{|c|c|c|}
\hline $\mathrm{H}_{2} \mathrm{O}+O\left({ }^{1} \mathrm{D}\right)$ & $\rightarrow$ & $2 O H$ \\
\hline$O\left({ }^{1} D\right)+H_{2}$ & $\rightarrow$ & $\mathrm{OH}+\mathrm{HO}_{2}$ \\
\hline$N_{2} O+O\left({ }^{1} D\right)$ & $\rightarrow$ & $\mathrm{N}_{2}+\mathrm{O}_{2}$ \\
\hline$N_{2} O+O\left({ }^{1} D\right)$ & $\rightarrow$ & $2 N O$ \\
\hline $\mathrm{CH}_{4}+\mathrm{O}\left({ }^{1} \mathrm{D}\right)$ & $\rightarrow$ & $\mathrm{CH}_{2} \mathrm{O}+\mathrm{H}_{2}$ \\
\hline$C H_{4}+O\left({ }^{1} D\right)$ & $\rightarrow$ & $\mathrm{CH}_{3} \mathrm{O}_{2}+\mathrm{OH}$ \\
\hline $\mathrm{H}_{2}+\mathrm{OH}$ & $\rightarrow$ & $\mathrm{H}_{2} \mathrm{O}+\mathrm{HO}_{2}$ \\
\hline $\mathrm{OH}+\mathrm{O}_{3}$ & $\rightarrow$ & $\mathrm{HO}_{2}+\mathrm{O}_{2}$ \\
\hline $\mathrm{OH}+\mathrm{O}$ & $\rightarrow$ & $\mathrm{O}_{2}+\mathrm{HO}_{2}$ \\
\hline $\mathrm{HO}_{2}+\mathrm{O}$ & $\rightarrow$ & $\mathrm{OH}+\mathrm{O}_{2}$ \\
\hline $\mathrm{HO}_{2}+\mathrm{O}_{3}$ & $\rightarrow$ & $\mathrm{OH}+2 \mathrm{O}_{2}$ \\
\hline $\mathrm{HO}_{2}+\mathrm{OH}$ & $\rightarrow$ & $\mathrm{H}_{2} \mathrm{O}+\mathrm{O}_{2}$ \\
\hline $\mathrm{HO}_{2}+\mathrm{HO}_{2}$ & $\rightarrow$ & $\mathrm{H}_{2} \mathrm{O}_{2}+\mathrm{O}_{2}$ \\
\hline $2 \mathrm{HO}_{2}+\mathrm{H}_{2} \mathrm{O}$ & $\rightarrow$ & $\mathrm{H}_{2} \mathrm{O}_{2}+\mathrm{O}_{2}+\mathrm{H}_{2} \mathrm{O}$ \\
\hline $\mathrm{H}_{2} \mathrm{O}_{2}+\mathrm{OH}$ & $\rightarrow$ & $\mathrm{H}_{2} \mathrm{O}+\mathrm{HO}_{2}$ \\
\hline $\mathrm{NO}+\mathrm{O}_{3}$ & $\rightarrow$ & $\mathrm{NO}_{2}+\mathrm{O}_{2}$ \\
\hline $\mathrm{NO}+\mathrm{OH}$ & $\rightarrow$ & HONO \\
\hline $\mathrm{NO}+\mathrm{HO}_{2}$ & $\rightarrow$ & $\mathrm{NO}_{2}+\mathrm{OH}$ \\
\hline $\mathrm{NO}_{2}+\mathrm{O}$ & $\rightarrow$ & $\mathrm{NO}+\mathrm{O}_{2}$ \\
\hline $\mathrm{NO}_{2}+\mathrm{O}_{3}$ & $\rightarrow$ & $\mathrm{NO}_{3}+\mathrm{O}_{2}$ \\
\hline $\mathrm{NO}_{2}+\mathrm{HO}_{2}$ & $\rightarrow$ & $\mathrm{HO}_{2} \mathrm{NO}_{2}$ \\
\hline $\mathrm{NO}_{3}+\mathrm{NO}_{2}$ & $\rightarrow$ & $\mathrm{N}_{2} \mathrm{O}_{5}$ \\
\hline $\mathrm{N}_{2} \mathrm{O}_{5}$ & $\rightarrow$ & $\mathrm{NO}_{3}+\mathrm{NO}_{2}$ \\
\hline $\mathrm{NO}_{2}+\mathrm{OH}$ & $\rightarrow$ & $\mathrm{HNO}_{3}$ \\
\hline $\mathrm{HONO}+\mathrm{OH}$ & $\rightarrow$ & $\mathrm{H}_{2} \mathrm{O}+\mathrm{NO}_{2}$ \\
\hline $\mathrm{HNO}_{3}+\mathrm{OH}$ & $\rightarrow$ & $\mathrm{H}_{2} \mathrm{O}+\mathrm{NO}+\mathrm{O}_{2}$ \\
\hline $\mathrm{HNO}_{3}+\mathrm{OH}$ & $\rightarrow$ & $\mathrm{H}_{2} \mathrm{O}+\mathrm{NO}_{2}+\mathrm{O}$ \\
\hline $\mathrm{HO}_{2} \mathrm{NO}_{2}$ & $\rightarrow$ & $\mathrm{HO}_{2}+\mathrm{NO}_{2}$ \\
\hline
\end{tabular}


123

TABLE III. Thermal reactions in the Box Model

$$
\begin{array}{lll}
\hline \mathrm{HO}_{2} \mathrm{NO}_{2}+\mathrm{OH} & \rightarrow \mathrm{H}_{2} \mathrm{O}+\mathrm{NO}_{2}+\mathrm{O}_{2} \\
\mathrm{CO}+\mathrm{OH} & \rightarrow \mathrm{HO}_{2} \\
\mathrm{CH}_{4}+\mathrm{OH} & \rightarrow \mathrm{CH}_{3} \mathrm{O}_{2}+\mathrm{H}_{2} \mathrm{O} \\
\mathrm{CH}_{2} \mathrm{O}+\mathrm{OH} & \rightarrow \mathrm{H}_{2} \mathrm{O}+\mathrm{HO}_{2}+\mathrm{CO} \\
\mathrm{CH}_{3} \mathrm{O}_{2}+\mathrm{O} & \rightarrow \mathrm{CH}_{2} \mathrm{O}+\mathrm{HO}_{2} \\
\mathrm{CH}_{2} \mathrm{O}+\mathrm{O} & \rightarrow \mathrm{HO}_{2}+\mathrm{OH}+\mathrm{CO} \\
\mathrm{CH}_{3} \mathrm{O}_{2}+\mathrm{HO}_{2} & \rightarrow \mathrm{CH}_{3} \mathrm{OOH}+\mathrm{O}_{2} \\
\mathrm{CH}_{3} \mathrm{O}_{2}+\mathrm{CH}_{3} \mathrm{O}_{2} & \rightarrow 2 \mathrm{CH}_{2} \mathrm{O}+1.4 \mathrm{HO}_{2} \\
\mathrm{CH}_{3} \mathrm{O}_{2}+\mathrm{NO} & \rightarrow \mathrm{HO}_{2}+\mathrm{CH}_{2} \mathrm{O}+\mathrm{NO}_{2} \\
\mathrm{CH}_{3} \mathrm{O}_{2}+\mathrm{NO} & \rightarrow \mathrm{CH}_{2} \mathrm{O}_{2} \mathrm{NO}_{2} \\
\mathrm{CH}_{3} \mathrm{O}_{2} \mathrm{NO} & \rightarrow \mathrm{CH}_{3} \mathrm{O}_{2}+\mathrm{NO}_{2} \\
\mathrm{CH}_{3} \mathrm{OOH}+\mathrm{OH} & \rightarrow \mathrm{CH}_{2} \mathrm{O}+\mathrm{H}_{2} \mathrm{O}+\mathrm{OH} \\
\mathrm{CH}_{3} \mathrm{OOH}+\mathrm{OH} & \rightarrow \mathrm{CH}_{3} \mathrm{O}_{2}+\mathrm{H}_{2} \mathrm{O}
\end{array}
$$

TABLE IV. Photolytic Reactions in the Box Model

$$
\begin{aligned}
& \mathrm{O}_{2}+h \mathrm{~V} \rightarrow 2 \mathrm{O} \\
& \mathrm{O}_{3}+h v \quad \rightarrow \mathrm{O}+\mathrm{O}_{2} \\
& \mathrm{O}_{3}+h v \quad \rightarrow O O\left({ }^{1} D\right)+O_{2} \\
& \mathrm{H}_{2} \mathrm{O}_{2}+h \mathrm{~V} \rightarrow 2 \mathrm{OH} \\
& \mathrm{NO}_{2}+h \mathrm{v} \rightarrow \mathrm{NO}+\mathrm{O} \\
& \mathrm{N}_{2} \mathrm{O}+h v \quad \rightarrow \quad \mathrm{N}_{2}+O\left({ }^{1} \mathrm{D}\right) \\
& \mathrm{NO}_{3}+h \mathrm{v} \rightarrow \mathrm{NO}_{2}+\mathrm{O} \\
& \mathrm{NO}_{3}+h \mathrm{~V} \rightarrow \mathrm{NO}+\mathrm{O}_{2} \\
& \mathrm{~N}_{2} \mathrm{O}_{5}+h \mathrm{~V} \quad \rightarrow \mathrm{NO}_{2}+\mathrm{NO}+\mathrm{O}_{2} \\
& \mathrm{~N}_{2} \mathrm{O}_{5}+h \mathrm{hv} \quad \rightarrow \quad 2 \mathrm{NO}_{2}+\mathrm{O} \text {. } \\
& \mathrm{N}_{2} \mathrm{O}_{5}+h \mathrm{~V} \rightarrow \mathrm{NO}_{2}+\mathrm{NO}+2 \mathrm{O} \\
& \mathrm{N}_{2} \mathrm{O}_{5}+h \mathrm{hV} \rightarrow 2 \mathrm{NO}+\mathrm{O}_{2}+\mathrm{O} \\
& \mathrm{HONO}+\mathrm{hv} \rightarrow \mathrm{OH}+\mathrm{NO} \\
& \mathrm{HNO}_{3}+h v \quad \rightarrow \mathrm{OH}+\mathrm{NO}_{2}
\end{aligned}
$$


124

TABLE IV. Photolytic Reactions in the Box Model

$$
\begin{array}{ll}
\hline \mathrm{HO}_{2} \mathrm{NO}_{2}+h \mathrm{v} & \rightarrow \mathrm{OH}+\mathrm{NO}+\mathrm{O}_{2} \\
\mathrm{HO}_{2} \mathrm{NO}_{2}+h \mathrm{v} & \rightarrow \mathrm{OH}+\mathrm{NO}_{2}+\mathrm{O} \\
\mathrm{HO}_{2} \mathrm{NO}_{2}+h \mathrm{hv} & \rightarrow \mathrm{HO}_{2}+\mathrm{NO}_{2} \\
\mathrm{CH}_{2} \mathrm{O}+h v & \rightarrow \mathrm{CO}+\mathrm{H}_{2} \\
\mathrm{CH}_{2} \mathrm{O}+h v & \rightarrow 2 \mathrm{HO}_{2}+\mathrm{CO} \\
\mathrm{CH}_{3} \mathrm{OOH}+h v & \rightarrow \mathrm{CH}_{2} \mathrm{O}+\mathrm{HO}_{2}+\mathrm{OH} \\
\mathrm{CH}_{3} \mathrm{O}_{2} \mathrm{NO}_{2}+h v & \rightarrow \mathrm{CH}_{3} \mathrm{O}_{2}+\mathrm{NO}_{2}
\end{array}
$$

2.0 3-D model

TABLE V. Reactions in the 3-D model (IMPACT)

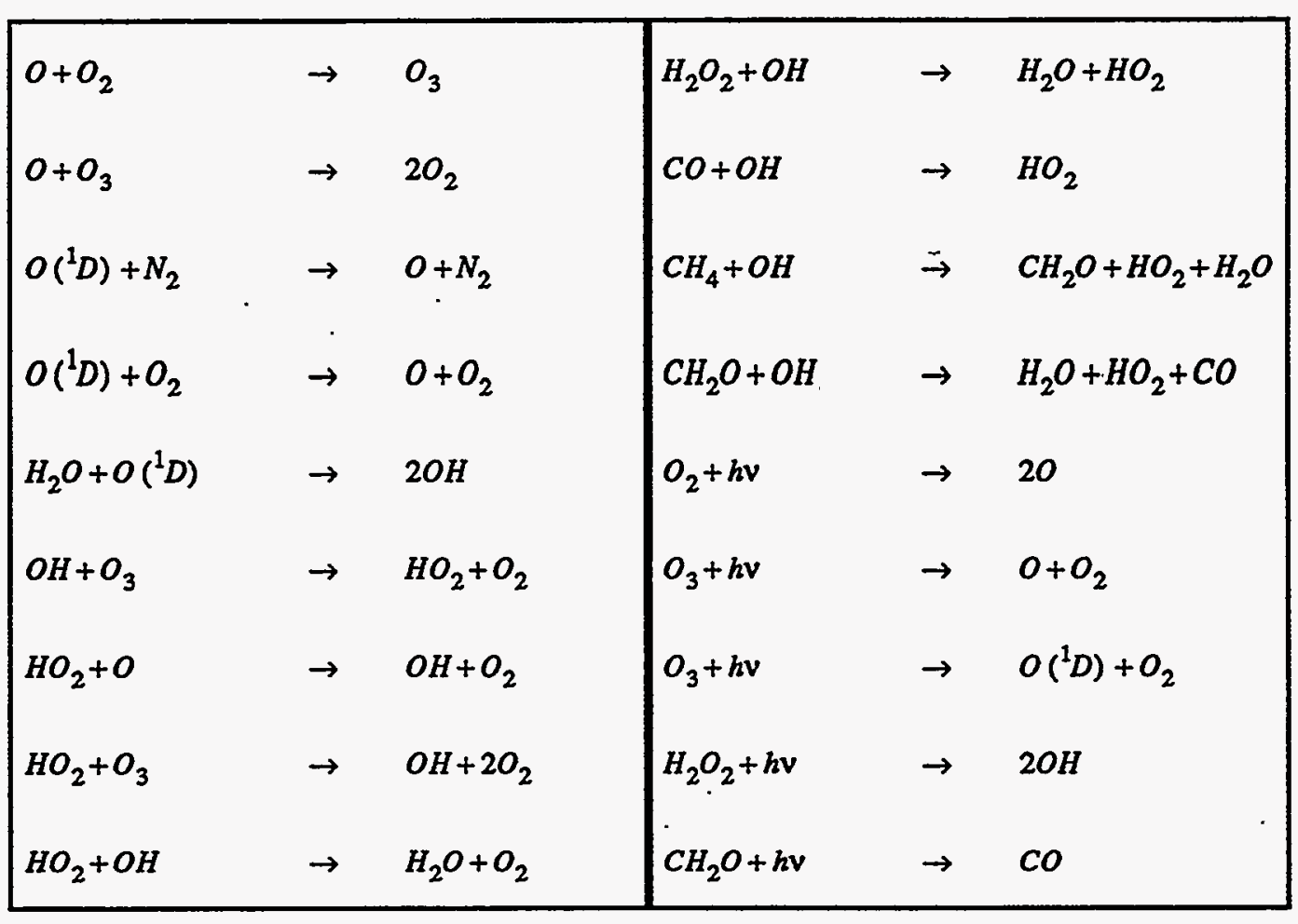




\begin{tabular}{|lll|lll}
\hline $\mathrm{HO}_{2}+\mathrm{HO}_{2}$ & $\rightarrow$ & $\mathrm{H}_{2} \mathrm{O}_{2}+\mathrm{O}_{2}$ & $\mathrm{CH}_{2} \mathrm{O}+h \mathrm{~V}$ & $\rightarrow$ & $2 \mathrm{HO}_{2}+\mathrm{CO}$ \\
$\mathrm{HO}_{2}+\mathrm{HO}_{2}+\mathrm{HO}_{2}$ & $\rightarrow$ & $\mathrm{H}_{2} \mathrm{O}_{2}+\mathrm{O}_{2}+\mathrm{H}_{2} \mathrm{O}$ & & & \\
\hline
\end{tabular}

\subsection{2-D Model}

\begin{tabular}{|c|c|c|}
\hline 1: & 03 & dynamic \\
\hline $2:$ & $\mathrm{N} 20$ & dynamic \\
\hline 3: & NO & dynamic \\
\hline 4: & NO2 & dynamic \\
\hline 5: & No3 & dynamic \\
\hline 6: & N205 & dynamic \\
\hline - $7:$ & HNO3 & dynamic \\
\hline 8: & $\mathrm{HO} 2 \mathrm{NO} 2$ & dynamic \\
\hline $9:$ & $\mathrm{H} 2 \mathrm{O}$ & dynamic \\
\hline 10: & $\mathrm{OH}$ & dynamic \\
\hline 11: & HO2 & dynamic \\
\hline 12: & $\mathrm{H} 2 \mathrm{O} 2$ & dynamic \\
\hline 13: & $\mathrm{H} 2$ & dynamic \\
\hline 14: & $\mathrm{CH} 4$ & dynamic \\
\hline 15: & $\mathrm{CH} 3 \mathrm{O} 2$ & dỹnamic \\
\hline 16: & $\mathrm{CH} 30 \mathrm{OH}$ & dynamic \\
\hline 17: & $\mathrm{CH} 2 \mathrm{O}$ & dynamic \\
\hline 18: & Co & dynamic \\
\hline 19: & $\mathrm{Cl}$ & dynamic \\
\hline $20:$ & Cl2 & dynamic \\
\hline 21: & ClO & dynamic \\
\hline $22:$ & OCIO & dynamic \\
\hline $23:$ & C1202 & dynamic \\
\hline $24:$ & $\mathrm{HCl}$ & dynamic \\
\hline 25: & HOCI & dynamic \\
\hline 26: & C10NO2 & dynamic \\
\hline $27:$ & $\mathrm{Br}$ & dynamic \\
\hline $28:$ & $\mathrm{BrCl}$ & dynamic \\
\hline 29: & Bro & dynamic \\
\hline $30:$ & $\mathrm{HBr}$ & dynamic \\
\hline 31: & HOBr & dynamic \\
\hline $32:$ & BrONO2 & dynamic \\
\hline 33: & $\mathrm{CH} 3 \mathrm{Cl}$ & dynamic \\
\hline $34:$ & $\mathrm{CH} 3 \mathrm{Br}$ & dynamic \\
\hline $35:$ & CECl3 & dynamic \\
\hline
\end{tabular}




$\begin{array}{rrr}\text { 36: } & \text { CF2C12 } & \text { dynamic } \\ \text { 37: } & \text { CFC113 } & \text { dynamic } \\ 38: & \text { CFC114 } & \text { dynamic } \\ \text { 39: } & \text { CFC115 } & \text { dynamic } \\ \text { 40: } & \text { HCFC22 } & \text { dynamic } \\ \text { 41: } & \text { CC14 } & \text { dynamic } \\ \text { 42: } & \text { CH3CCI3 } & \text { dynamic } \\ \text { 43: } & \mathrm{CF} 3 \mathrm{Br} & \text { dynamic } \\ \text { 44: } & \mathrm{CF} 2 \mathrm{CIBr} & \text { dynamic } \\ \text { 1: } & \mathrm{O} & \text { steadystate } \\ \text { 2: } & \mathrm{OID} & \text { steadystate } \\ 3: & \mathrm{N} & \text { steadystate } \\ \text { 4: } & \mathrm{H} & \text { steadystate } \\ \text { 1: } & \mathrm{N} 2 & \text { constant } \\ \text { 2: } & \mathrm{O} 2 & \text { constant }\end{array}$

Thermal reactions:

$$
\begin{aligned}
& \text { 1: } 0+02=03 \\
& \text { 2: } 0+03=2 * 02 \\
& \text { 3: } \mathrm{O} 1 \mathrm{D}+\mathrm{N} 2=\mathrm{O}+\mathrm{N} 2 \\
& \text { 4: } \mathrm{O} 1 \mathrm{D}+\mathrm{O} 2=0+02 \\
& \text { 5: } 01 D+03=2 * 02 \\
& \text { 6: } 01 D+03=02+2 * 0 \\
& \text { 7: } \mathrm{H} 2 \mathrm{O}+\mathrm{O} 1 \mathrm{D}=2 * \mathrm{OH} \\
& \text { 8: } \mathrm{H} 2+\mathrm{O} 1 \mathrm{D}=\mathrm{OH}+\mathrm{H} \\
& \text { 9: } \mathrm{N} 2 \mathrm{O}+\mathrm{O} 1 \mathrm{D}=\mathrm{N} 2+\mathrm{O} 2 \\
& \text { 10: } \mathrm{N} 2 \mathrm{O}+\mathrm{O} \mathrm{OD}=2 * \mathrm{NO} \\
& \text { 11: } \mathrm{CH} 4+\mathrm{O} 1 \mathrm{D}=\mathrm{CH} 3 \mathrm{O} 2+\mathrm{OH} \\
& \text { 12: } \mathrm{CF} 2 \mathrm{Cl} 2+\mathrm{O} 1 \mathrm{D}=2 * \mathrm{Cl} \\
& \text { 13: } \mathrm{CFC} 113+01 \mathrm{O}=3 * \mathrm{Cl} \\
& \text { 14: CFC114. + O1D }=2 * \mathrm{Cl} \\
& \text { 15: } \mathrm{CFC} 115+\text { O1D }=\mathrm{Cl} \text {. } \\
& \text { 16: } \mathrm{HCFC} 22+\mathrm{O} 1 \mathrm{D}=\mathrm{Cl} \\
& \text { 17: } \mathrm{H}+\mathrm{O} 2=\mathrm{HO} 2 \\
& \text { 18: } \mathrm{H}+03=\mathrm{OH}+\mathrm{O} 2 \\
& \text { 19: } \mathrm{OH}+\mathrm{H} 2=\mathrm{H} 2 \mathrm{O}+\mathrm{H} \\
& \text { 20: } \mathrm{OH}+\mathrm{O} 3=\mathrm{HO} 2+\mathrm{O} 2 \\
& \text { 21: } \mathrm{OH}+\mathrm{O}=\mathrm{O} 2+\mathrm{H} \\
& \text { 22: } \mathrm{OH}+\mathrm{OH}=\mathrm{H} 2 \mathrm{O}+\mathrm{O} \\
& \text { 23: } \mathrm{HO} 2+\mathrm{O}=\mathrm{OH}+\mathrm{O} 2 \\
& \text { 24: } \mathrm{HO} 2+\mathrm{O} 3=\mathrm{OH}+2 * 02 \\
& \text { 25: } \mathrm{H}+\mathrm{HO} 2=2 * \mathrm{OH} \\
& \text { 26: } \mathrm{HO} 2+\mathrm{OH}=\mathrm{H} 2 \mathrm{O}+\mathrm{O} 2 \\
& \text { 27: } \mathrm{HO} 2+\mathrm{HO} 2=\mathrm{H} 2 \mathrm{O} 2+\mathrm{O} 2 \\
& \text { 28: } \mathrm{HO} 2+\mathrm{HO} 2+\mathrm{H} 2 \mathrm{O}=\mathrm{H} 2 \mathrm{O} 2+\mathrm{O} 2+\mathrm{H} 2 \mathrm{O} \\
& \text { 29: } \mathrm{H} 2 \mathrm{O} 2+\mathrm{OH}=\mathrm{H} 2 \mathrm{O}+\mathrm{HO} 2
\end{aligned}
$$




$$
\begin{aligned}
& 30: \mathrm{N}+\mathrm{O} 2=\mathrm{NO}+\mathrm{O} \\
& 31: \mathrm{N}+\mathrm{NO}=\mathrm{N} 2+\mathrm{O} \\
& \text { 32: } \mathrm{NO}+03=\mathrm{NO} 2+02 \\
& \text { 33: } \mathrm{NO}+\mathrm{HO} 2=\mathrm{NO} 2+\mathrm{OH} \\
& \text { 34: } \mathrm{NO} 2+\mathrm{O}=\mathrm{NO}+\mathrm{O} 2 \\
& \text { 35: } \mathrm{NO} 2+\mathrm{O} 3=\mathrm{NO} 3+\mathrm{O} 2 \\
& \text { 36: } \mathrm{NO} 2+\mathrm{HO} 2=\mathrm{HO} 2 \mathrm{NO} 2 \\
& \text { 37: } \mathrm{NO} 3+\mathrm{NO}=2 \text { * } \mathrm{NO} 2 \\
& \text { 38: } \mathrm{NO} 3+\mathrm{NO} 2=\mathrm{N} 205 \\
& \text { 39: } \mathrm{N} 205=\mathrm{NO} 2+\mathrm{NO} 3 \\
& \text { 40: } \mathrm{N} 205=2{ }^{*} \mathrm{HNO} 3 \\
& \text { 41: } \mathrm{NO} 2+\mathrm{OH}=\mathrm{HNO} 3 \\
& \text { 42: } \mathrm{HNO} 3+\mathrm{OH}=\mathrm{H} 2 \mathrm{O}+\mathrm{NO} 3 \\
& \text { 43: } \mathrm{HO} 2 \mathrm{NO} 2=\mathrm{HO} 2+\mathrm{NO} 2 \\
& \text { 44: } \mathrm{HO} 2 \mathrm{NO} 2+\mathrm{OH}=\mathrm{H} 2 \mathrm{O}+\mathrm{NO} 2+\mathrm{O} 2 \\
& \text { 45: } \mathrm{Cl}+\mathrm{O} 3=\mathrm{ClO}+\mathrm{O} 2 \\
& \text { 46: } \mathrm{Cl}+\mathrm{H} 2=\mathrm{HCl}+\mathrm{H} \\
& \text { 47: } \mathrm{Cl}+\mathrm{H} 2 \mathrm{O} 2=\mathrm{HCl}+\mathrm{HO} 2 \\
& \text { 48: } \mathrm{Cl}+\mathrm{HO} 2=\mathrm{HCl}+\mathrm{O} 2 \\
& \text { 49: } \mathrm{Cl}+\mathrm{HO} 2=\mathrm{OH}+\mathrm{ClO} \\
& \text { 50: } \mathrm{ClO}+\mathrm{O}=\mathrm{Cl}+\mathrm{O} 2 \\
& \text { 51: } \mathrm{ClO}+\mathrm{OH}=\mathrm{HO} 2+\mathrm{Cl} \\
& \text { 52: } \mathrm{ClO}+\mathrm{OH}=\mathrm{HCl}+\mathrm{O} 2 \\
& \text { 53: } \mathrm{ClO}+\mathrm{HO} 2=\mathrm{O} 2+\mathrm{HOCl} \\
& 54: \mathrm{ClO}+\mathrm{NO}=\mathrm{NO} 2+\mathrm{Cl} \\
& \text { 55: } \mathrm{ClO}+\mathrm{NO} 2=\mathrm{ClONO} 2 \\
& \text { 56: } \mathrm{ClO}+\mathrm{ClO}=2 * \mathrm{Cl}+\mathrm{O} 2 \\
& \text { 57: } \mathrm{ClO}+\mathrm{ClO}=\mathrm{Cl} 2+\mathrm{O} 2 \\
& \text { 58: } \mathrm{ClO}+\mathrm{ClO}=\mathrm{Cl}+\mathrm{OClO} \\
& \text { 59: } \mathrm{ClO}+\mathrm{ClO}=\mathrm{Cl} 202 \\
& \text { 60: } 0 \mathrm{ClO}+\mathrm{Cl}=2{ }^{*} \mathrm{ClO} \\
& \text { 61: } 0 \mathrm{ClO}+\mathrm{O}=\mathrm{O} 2+\mathrm{ClO} \\
& \text { 62: } \mathrm{OClO}+\mathrm{OH}=\mathrm{HOCl}+\mathrm{O} 2 \\
& \text { 63: } \mathrm{OClO}+\mathrm{NO}=\mathrm{NO} 2+\mathrm{ClO} \\
& \text { 64: } \mathrm{Cl} 2 \mathrm{O} 2+\mathrm{Cl}=\mathrm{Cl} 2+\mathrm{Cl}+\mathrm{O} 2 \\
& \text { 65: } \mathrm{C} 1202=2{ }^{*} \mathrm{ClO} \\
& \text { 66: } \mathrm{HCl}+\mathrm{OH}=\mathrm{H} 2 \mathrm{O}+\mathrm{Cl} \\
& \text { 67: } \mathrm{HOCl}+\mathrm{OH}=\mathrm{H} 2 \mathrm{O}+\mathrm{ClO} \\
& \text { 68: } \mathrm{ClONO} 2+\mathrm{O}=\mathrm{ClO}+\mathrm{NO} 3 \\
& \text { 69: } \mathrm{ClONO} 2+\mathrm{OH}=\mathrm{HOCl}+\mathrm{NO} 3 \\
& \text { 70: } \mathrm{ClONO} 2+\mathrm{Cl}=\mathrm{Cl} 2+\mathrm{NO} 3 \\
& \text { 71: } \mathrm{ClONO} 2=\mathrm{HOCl}+\mathrm{HNO} 3 \\
& \text { 72: } \mathrm{Br}+03=\mathrm{BrO}+02 \\
& \text { 73: } \mathrm{Br}+\mathrm{HO} 2=\mathrm{HBr}+02 \\
& \text { 74: } \mathrm{Br}+\mathrm{CH} 2 \mathrm{O}=\mathrm{HBr}+\mathrm{HO} 2+\mathrm{CO} \\
& \text { 75: } \mathrm{BrO}+\mathrm{O}=\mathrm{Br}+\mathrm{O} 2 \\
& \text { 76: } \mathrm{BrO}+\mathrm{HO} 2=\mathrm{HOBr}+\mathrm{O} 2
\end{aligned}
$$




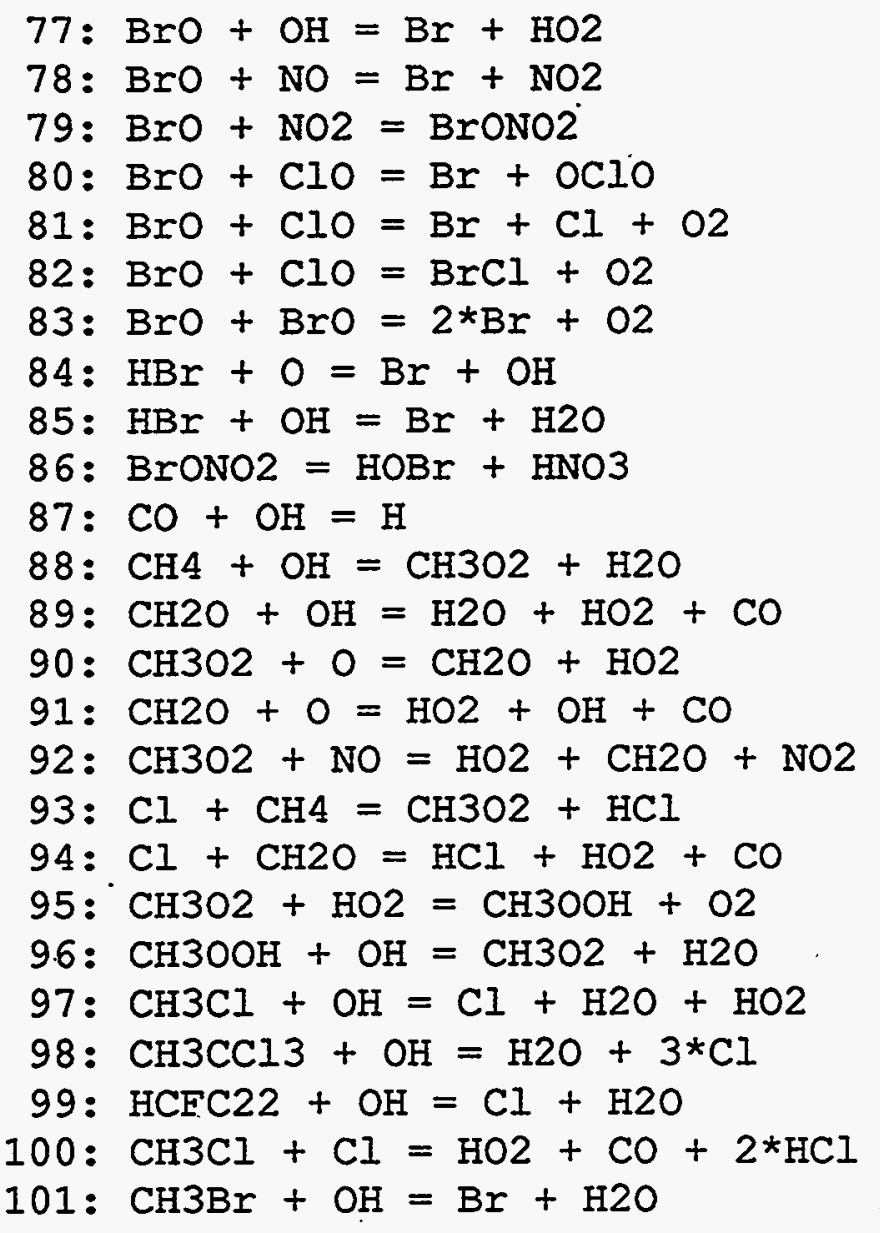

Photolytic reactions:

1: $02+h v=2 * 0$

2: $03+\mathrm{hv}=0+02$

3: $03+\mathrm{hv}=01 \mathrm{D}+02$

4: $\mathrm{HO} 2+\mathrm{hv}=\mathrm{OH}+\mathrm{O}$

5: $\mathrm{H} 2 \mathrm{O} 2+\mathrm{hV}=2 * \mathrm{OH}$

6: $\mathrm{H} 2 \mathrm{O}+\mathrm{hV}=\mathrm{H}+\mathrm{OH}$

7: $\mathrm{NO} 2+\mathrm{hv}=\mathrm{NO}+\mathrm{O}$

8: $\mathrm{N} 20+\mathrm{hv}=\mathrm{N} 2+01 \mathrm{D}$

9: $\mathrm{NO}+\mathrm{hV}=\mathrm{N}+\mathrm{O}$

10: $\mathrm{NO} 3+\mathrm{hv}=\mathrm{NO} 2+\mathrm{O}$

11: $\mathrm{NO} 3+\mathrm{hv}=\mathrm{NO}+\mathrm{O} 2$

12: $\mathrm{N} 205+\mathrm{hv}=\mathrm{NO} 2+\mathrm{NO} 3$

13: $\mathrm{N} 205+\mathrm{hv}=\mathrm{NO}+\mathrm{O}+\mathrm{NO} 3$

14: $\mathrm{HNO} 3+\mathrm{hv}=\mathrm{OH}+\mathrm{NO} 2$

15: $\mathrm{HO} 2 \mathrm{NO} 2+\mathrm{hv}=\mathrm{OH}+\mathrm{NO} 3$

16: $\mathrm{HO} 2 \mathrm{NO} 2+\mathrm{hv}=\mathrm{HO} 2+\mathrm{NO} 2$

17: $\mathrm{Cl} 2+\mathrm{hv}=2 * \mathrm{Cl}$

18: $\mathrm{ClO}+\mathrm{hV}=\mathrm{Cl}+\mathrm{O}$ 